Bull. Soc. math. France

132 (1), 2004, p. 1-54

\title{
INTÉGRATION MOTIVIQUE SUR \\ LES SCHÉMAS FORMELS
}

\author{
PAR Julien SEbag
}

\begin{abstract}
RÉSUMÉ. - Nous généralisons la théorie de l'intégration motivique au cadre des schémas formels. Nous définissons et étudions l'anneau booléen des ensembles mesurables, la mesure motivique, l'intégrale motivique et nous démontrons un théorème de changement de variables pour cette intégrale.
\end{abstract}

Abstract (Motivic Integration on Formal Schemes). - We generalize the theory of motivic integration on formal schemes. In particular, we define and study the boolean ring of mesurable subsets, the motivic measure, the motivic integral and we prove a theorem of change of variables for this integral.

\section{Introduction}

1.1. Dans leurs articles [10] et [12] ( $c f$. également [11]), Denef et Loeser développent et étudient la théorie de l'intégration motivique pour les variétés algébriques sur un corps de caractéristique 0 , introduite par Kontsevich lors d'un séminaire à Orsay ( $c f$. [18]). Dans [20], Looijenga étend la construction à la catégorie des variétés algébriques sur un anneau de séries formelles de corps résiduel de caractéristique 0 . Cette nouvelle théorie de l'intégration se révèle être un outil puissant dans l'étude de la géométrie birationnelle des variétés algébriques sur un corps $k$ de caractéristique 0 . Rappelons rapidement les grandes étapes de la construction de ces intégrales et les idées sous-jacentes

Texte reçu le 14 janvier 2002, révisé le 16 décembre 2002, accepté le 7 mars 2003

Julien Sebag, Université de Bordeaux, AZX, UMR 5465 CNRS, 351, cours de la libération, 33405 Talence Cedex (France) • E-mail : Julien.Sebag@math.u-bordeaux.fr Classification mathématique par sujets (2000). — 14-XX, 28-XX.

Mots clefs. - Géométrie algébrique, géométrie formelle, intégration motivique. 
à quelques-uns des résultats obtenus par cette théorie. Si $X$ est une variété algébrique sur un corps $k$ de caractéristique 0 , on lui associe, de manière fonctorielle, un pro- $k$-schéma, qui est encore un schéma (non localement de type fini en général), le schéma des arcs sur $X$, noté $L(X)$. Sur ce pro- $k$-schéma, on définit une mesure $\mu_{X}$ à valeurs dans un anneau de motifs virtuels. Cette mesure motivique est un analogue géométrique de la mesure $p$-adique sur les variétés différentielles $p$-adiques. Les intégrales définies à partir de cette mesure vérifient alors un théorème de changement de variables pour les $k$-morphismes de schémas $h: Y \rightarrow X$ propres et birationnels, qui permet de calculer les intégrales sur $L(X)$ en fonction d'intégrales sur $L(Y)$. C'est essentiellement par ce principe que l'on peut construire de nouveaux invariants algébriques (à valeurs dans cet anneau de motifs virtuels, ou plus exactement dans le séparé complété de cet anneau pour une filtration) et que l'on peut, par exemple, (re)-démontrer le théorème de Batyrev qui affirme que deux variétés de Calabi-Yau, birationnellement équivalentes, ont mêmes nombres de Hodge et même structure de Hodge.

1.2. Dans cet article, nous généralisons cette théorie de l'intégration motivique aux schémas formels sttf sur le spectre formel d'un anneau de valuation discrète complet $R$, de corps résiduel $k$ parfait. En particulier, le corps $k$ peut être de caractéristique positive. L'absence du morphisme d'inclusion $k \hookrightarrow R$, dans le cas où $R$ est un anneau d'inégales caractéristiques, nous conduit à définir un analogue du schéma des arcs d'une variété $X$. Si $X$ est un $R$-schéma formel sttf, le schéma de Greenberg $\operatorname{Gr}(X)$ joue ce rôle. Il est important de noter que, dans le cas où $R=k[[t]]$, avec $k$ de caractéristique 0 , et $\widehat{X}$ est le complété $\pi$-adique d'une $k$-variété algébrique vue sur $k[[t]]$, les deux $k$-schémas $\operatorname{Gr}(\widehat{X})$ et $L(X)$ sont canoniquement isomorphes. En outre, pour tout $R$-schéma formel sttf, si $F$ est une extension parfaite de $k$, les $F$-points de $\operatorname{Gr}(X)$ s'interprètent naturellement comme des points de la fibre générique $X_{K}$ de $X$. Comme dans le cas du schéma des arcs, nous définissons l'anneau booléen des ensembles mesurables, qui sont des «approximations » par certaines parties constructibles de $\operatorname{Gr}(X)$ élémentaires, que l'on appelle cylindres. Rappelons que le produit fibré au-dessus de $k$ induit sur le groupe de Grothendieck de la catégorie des $k$-variétés une structure d'anneau (puisque $k$ est parfait). On note $M_{k}$ le localisé de cet anneau par rapport à la classe de la droite affine. La définition des ensembles mesurables nous permet de construire une mesure $\mu_{X}$ à valeurs dans un complété de l'anneau $M_{k}$. De manière analogue aux théories classiques d'intégration, les ensembles mesurables possèdent des propriétés de stabilité par image directe et inverse sous certains $R$-morphismes $h: Y \rightarrow X$, que l'on nomme tempérés, et qui correspondent aux morphismes propres et birationnels du cas algébrique. Nous construisons l'intégrale motivique des fonctions intégrables. Parmi celles-ci, les fonctions exponentiellement intégrables permettent 
d'exprimer, de manière naturelle, des phénomènes géométriques comme l'appartenance à un sous- $R$-schéma formel fermé, la lissité d'un $R$-morphisme de schémas formels... et jouent donc un rôle central dans cette théorie. Enfin, nous démontrons deux théorèmes de changement de variables du même type que celui énoncé dans [10] ou [12].

1.3. Les principaux résultats de ce travail sont utilisés de manière fondamentale dans [19], qui, en se plaçant du point de vue rigide, déduit, des constructions et théorèmes de cet article, certaines applications en géométrie birationnelle des dégénérescences des variétés algébriques et rigides. En particulier, la théorie que l'on développe ici apparaît, au regard des résultats de [19], comme l'analogue (ou la généralisation) de l'intégration motivique usuelle et de l'intégration $p$ adique. Deux exemples le confirment. Soit $X_{K}$ la fibre générique d'un $R$-schéma formel $X$ sttf.

1) Si $\omega$ est une forme jauge sur $X_{K}$, son intégrale converge déjà dans $M_{k}$. En outre, nous démontrons que sa classe dans $M_{k} /(\mathbf{L}-1) M_{k}$ ne dépend que de $X_{K}$ et non de la forme jauge choisie pour calculer cette intégrale. Cet élément de $M_{k}$, noté $\lambda\left(X_{K}\right)$, est égal à la classe de la fibre spéciale de tout modèle de Néron faible de $X_{K}$. Il faut également remarquer que, quand $K$ est une extension finie de $\mathbf{Q}_{p}, \lambda\left(X_{K}\right)$ se spécialise en l'invariant de Serre [25] évalué sur la variété analytique $p$-adique «naïve » sous-jacente à $X_{K}$ (cf. corollaire 4.6.3 de [19]).

2) Si $X_{K}$ est l'espace analytique associé une variété de Calabi-Yau sur $K$, et si $X_{K}$ admet un $R$-modèle propre et lisse, l'intégrale calculée pour un générateur de $\Omega_{X_{K}}^{d}$ est égale à la classe de la fibre spéciale de ce modèle dans $M_{k}$. En particulier, les classes des fibres spéciales de deux tels modèles coïncident dans $M_{k}$, ce qui peut être interprété comme l'analogue du résultat de Batyrev [2] pour les variétés de Calabi-Yau.

1.4. Cet article est organisé de la manière suivante : le chapitre 2 rappelle les bases de la géométrie formelle. Le foncteur de Greenberg est construit et étudié au chapitre 3 . Les chapitres 4,5 , et 6 développent, de manière assez complète, les notions et les propriétés de la mesure motivique, des cylindres et des ensembles mesurables. Le chapitre 7 est consacré à l'étude de la stabilité de la mesurabilité par image directe et inverse. Au chapitre 8, nous énonçons et démontrons deux théorèmes de changement de variables.

Nous remercions François Loeser de nous avoir proposé ce sujet et de l'aide qu'il nous a apportée lors des discussions que nous avons eues ensemble. Nous souhaitons également remercier Antoine Chambert-Loir de son aide lors de la rédaction de cet article et de ses commentaires sur une première version de ce texte. Plus largement, nous les remercions pour l'attention qu'ils nous ont manifestée depuis quelques années déjà. 


\section{Préliminaires}

\subsection{Les premières notions de géométrie formelle.}

2.1.1. La définition des schémas formels. — Un $\mathbb{D}$-schéma formel désignera toujours un $\mathbb{D}$-schéma formel topologiquement de type fini, ce que l'on notera $t t f$. Parfois l'hypothèse de séparation sera nécessaire et nos $\mathbb{D}$-schémas formels $t t f$ seront séparés, ce que l'on notera sttf ( $c f .[16, \S 10])$.

On notera $\underline{\text { Form }}_{/ \mathbb{D}}^{\text {ttf }}$ et $\underline{\text { Form }}^{\text {sttf }}$ les catégories correspondantes.

Un objet de $\underline{\text { Form }}_{/ \mathbb{D}}^{\text {ttf }}$ est un espace localement annelé $\left(X, O_{X}\right)$ en $R$-algèbres topologiques, qui induit la donnée, pour tout $n \geq 0$, d'un $R_{n}$-schéma

$$
X_{n}=\left(X, O_{X} \otimes_{R} R_{n}\right) .
$$

Le $k$-schéma $X_{0}$ est appelé fibre spéciale du $\mathbb{D}$-schéma formel $X$. En tant qu'espaces topologiques, $X$ et $X_{0}$ sont isomorphes et $O_{X}:=\lim _{X_{X_{n}}}$. On a

$$
X_{n}=X_{n+1} \otimes_{R_{n+1}} R_{n}
$$

et $X$ est canoniquement isomorphe à la limite inductive des schémas $X_{n}$ dans la catégorie des espaces localement annelés. En outre, un tel objet est localement isomorphe à un $\mathbb{D}$-schéma formel affine $\operatorname{Spf} A$, où $A$ est une $R$-algèbre $\pi$-adique, topologiquement isomorphe à un quotient de l'anneau des séries formelles restreintes $R\left\{T_{1}, \ldots, T_{N}\right\}$.

Si $X$ et $Y$ sont deux $\mathbb{D}$-schémas formels $t t f$, on note $\operatorname{Hom}_{\mathbb{D}}(Y, X)$ l'ensemble des $\mathbb{D}$-morphismes de schémas formels :

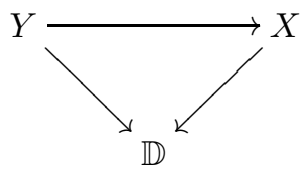

i.e. l'ensemble des morphismes entre les $\mathbb{D}$-espaces localement annelés sousjacents. Autrement dit, $\underline{\text { Form }}_{/ \mathbb{D}}^{\text {ttf }}$ est une sous-catégorie pleine de la catégorie des espaces localement annelés sur $\mathbb{D}$. Localement de tels morphismes sont simplement des $R$-morphismes continus d'algèbres topologiques entre les anneaux des sections globales. On peut montrer ( $c f$. prop. 10.6 .9 de [16]) que l'application canonique $\operatorname{Hom}_{\mathbb{D}}(Y, X) \rightarrow \lim _{\operatorname{Hom}_{n}}\left(Y_{n}, X_{n}\right)$ est une bijection.

REMARQUe 2.1.2. - Soit

$$
h: Y=\operatorname{Spf} R\left\{x_{1}, \ldots, x_{m}\right\} / I \rightarrow X=\operatorname{Spf} R\left\{x_{1}, \ldots, x_{n}\right\} / J
$$

un $\mathbb{D}$-morphisme de schémas formels. Dans ce cas, la donnée de $h$ est équivalente à celle d'un $R$-morphisme d'algèbres. En effet, la $R$-linéarité impose que le morphisme entre les anneaux des sections globales est continu pour les topologies $\pi$-adiques considérées.

TOME $132-2004-\mathrm{N}^{\mathrm{O}} 1$ 
La catégorie $\underline{\text { Form }}_{/ \mathbb{D}}^{\text {sttf }}$ est simplement la sous-catégorie pleine de $\underline{\text { Form }}_{/ \mathbb{D}}^{\text {ttf }}$, dont les objets sont séparés $(c f .[16, \S 10])$. Un $\mathbb{D}$-schéma formel sttf $X$ est dit admissible s'il est plat sur $\mathbb{D}$ (ce qui est équivalent au fait que le faisceau $O_{X}$ soit sans $\pi$-torsion). On notera $\underline{\text { Form }}_{/ \mathbb{D}}^{\text {Adm }}$ cette catégorie, qui est une sous-catégorie pleine de $\underline{\text { Form }}^{\text {sttf }}$.

Si $X$ est un $\mathbb{D}$-schéma formel, on notera $X_{\text {red }}$ le sous-schéma formel fermé et réduit de $X$ défini par le faisceau d'idéaux $\sqrt{0}$.

Remarque 2.1.3. — Si $X$ est un $\mathbb{D}$-schéma formel admissible et si $X_{0}$ est un $k$-schéma réduit, alors $X$ est réduit.

2.1.4. La notion de fibre générique d'un schéma formel. — Soient $X$ un $\mathbb{D}$ schéma formel ttf et $Z \hookrightarrow X$ un sous-schéma fermé de $X$, défini par le sousfaisceau $A \subset O_{X}$. On appelle éclatement admissible sur $X$ de centre $Z$ la donnée d'un $\mathbb{D}$-schéma formel $X^{\prime}$ et d'un $\mathbb{D}$-morphisme de schémas formels $\sigma: X^{\prime} \rightarrow X$ tel que $A \cdot O_{X^{\prime}}$ est inversible et vérifiant la propriété universelle suivante : si $\psi: Y \rightarrow X$ est un $\mathbb{D}$-morphisme de schémas formels tel que $A \cdot O_{Y}$ soit inversible sur $Y$, alors il existe un $\mathbb{D}$-morphisme $\psi^{\prime}: Y \rightarrow X^{\prime}$ tel que $\psi=\sigma \circ \psi^{\prime}$. Si $X$ est sttf (resp. admissible), le $\mathbb{D}$-schéma formel $X^{\prime}$ l'est aussi (cf. [4], $§ 2$ et spécialement le lemme 2.2).

La localisation de la catégorie Form $\underline{\mathrm{Forf}}_{/ \mathbb{D}}^{\mathrm{tf}}\left(\operatorname{resp} . \underline{\mathrm{Form}}_{/ \mathbb{D}}^{\mathrm{stff}}\right)$ par rapport aux éclatements admissibles est équivalente à la catégorie des $K$-espaces rigides de type fini et quasi-séparés (resp. séparés) au sens de Kiehl, que l'on notera $\underline{\mathrm{Rig}_{\text {qsqc }}^{\text {q }}}$ (resp. $\left.\underline{\mathrm{Rig}}_{/ K}^{\mathrm{sqc}}\right)(c f .[23]$ et $[4$, th. 4.1]). On peut remarquer que la localisée de $\underline{\text { Form }}_{/ \mathbb{D}}^{\mathrm{Adm}}$ par rapport aux éclatements admissibles est équivalente à $\underline{\mathrm{Rig}} / K$ sqc

Par analogie avec le cas de schémas usuels, on appellera fibre générique l'image d'un $\mathbb{D}$-schéma formel $t t f$ par le foncteur de localisation. Ce foncteur sera noté rig et si $X \in \underline{\text { Form }}_{/ \mathbb{D}}^{\text {ttf }}$ on notera $X_{\text {rig }}$ son image par le foncteur rig (ou parfois $\left.X_{K}\right)$. De même, si $f: Y \rightarrow X$ est un $\mathbb{D}$-morphisme de schémas formels, on notera $f_{\text {rig }}$ son image par le foncteur rig.

2.1.5. La notion de dimension d'un schéma formel. — Soit $X$ un $\mathbb{D}$-schéma formel. On appelle dimension de $X$ l'entier $\operatorname{dim} X$ défini comme la dimension de la fibre spéciale $X_{0}$ de $X$. En particulier, si $X:=\operatorname{Spf} A$ est plat sur $R$, on a la relation

$$
\operatorname{dim} X=\operatorname{dim}_{\mathrm{K}} A-1
$$

où $\operatorname{dim}_{\mathrm{K}} A$ est la dimension de Krull de l'anneau $A$.

Soit $X_{K}$ un $K$-espace rigide quasi-séparé et quasi-compact. On appelle dimension de $X_{K}$ et l'on note $\operatorname{dim} X_{K}$ l'entier naturel défini comme la borne supérieure des entiers $\operatorname{dim}_{K} O_{X_{K}, x}$ pour $x \in X_{K}$.

BUlletin DE LA SOCiÉtÉ MATHÉmATiQUe DE FRANCE 
LEMME 2.1.6. - Si $X_{K}=\operatorname{Spm} A$ est un $K$-espace rigide affinö̈de, la dimension de $X_{K}$ est égale à la dimension de Krull de $A$.

Démonstration. - Ce lemme résulte de la proposition 7.3.8 de [3] qui assure que l'anneau $O_{X_{K}, x}$ et le localisé de $A$ en $x, A_{x}$, ont même dimension de Krull.

Le lemme suivant, démontré par Oesterlé dans [21] (cf. lemme 1, §3), permet de relier ces deux notions.

LEMME 2.1.7. - Soient $R$ un anneau de valuation discrète complet, $k$ son corps résiduel et $K$ son corps des fractions. Notons $A$ l'anneau $R\left\{T_{1}, \ldots, T_{N}\right\}$ des séries formelles restreintes à coefficients dans $R, A_{K}=A \otimes_{R} K=$ $K\left\{T_{1}, \ldots, T_{N}\right\}$ et $A_{0}=A \otimes_{R} k=k\left[T_{1}, \ldots, T_{N}\right]$. Soit $I_{K}$ un idéal de $A_{K}$. Notons $I:=I_{K} \cap A$ et $I_{0}:=I \otimes_{R} k$. Supposons que l'anneau $A_{K} / I_{K}$ soit équidimensionnel, de dimension de Krull d. Alors l'anneau $A_{0} / I_{0}$ est équidimensionnel, de dimension de Krull d. On a $I_{K}=A_{K}$ si et seulement si $I_{0}=A_{0}$.

Corollaire 2.1.8. - Soit $X$ un $\mathbb{D}$-schéma formel sttf de fibre générique $X_{K}$. Si $X_{K}$ est équidimensionnelle de dimension d, alors $X_{0}$ est équidimensionnel de dimension $d$.

Démonstration. - On peut supposer que $X$ est affine. Dans ce cas le corollaire découle du lemme 2.1.7.

Un $\mathbb{D}$-schéma formel sttf est dit de dimension relative $d$ (resp. purement de dimension relative $d$ ) si sa fibre générique $X_{K}$ est de dimension $d$ (resp. équidimensionnelle de dimension $d$ ).

2.1.9. La notion de lissité pour les schémas formels. - Un $\mathbb{D}$-morphisme de schémas formels $f: Y \rightarrow X$ est lisse au point $y \in Y_{0}$ de dimension relative $d$ si :

(i) $f$ est plat en $y$;

(ii) le $k$-morphisme induit $f_{0}: Y_{0} \rightarrow X_{0}$ est lisse en $y$ de dimension relative $d$, au sens usuel.

D'après le lemme 1.2 de [4], il revient au même de demander que pour tout $n \in \mathbf{N}$, le morphisme induit $f_{n}: Y_{n} \rightarrow X_{n}$ soit lisse de dimension relative $d$ en $y$. On dit qu'un morphisme est lisse s'il est lisse en tout point $y \in Y_{0}$.

Un $\mathbb{D}$-schéma formel $X$ est dit lisse (en $x \in X_{0}$ ) si le morphisme structural $X \rightarrow \mathbb{D}$ est lisse (en $x \in X_{0}$ ) au sens précédent.

Soit $X$ un $\mathbb{D}$-schéma formel ttf de dimension $d$. On note $X_{\text {sing }}$ l'unique sous$\mathbb{D}$-schéma formel fermé réduit défini par le radical du $d$-ième idéal de Fitting de $\Omega_{X / \mathbb{D}}^{1}$, qui est le faisceau des formes différentielles continues (cf. [5], $\S 1$ ). En particulier, un $\mathbb{D}$-schéma formel ttf plat est lisse en $x \in X_{0}$ (resp. lisse) si et seulement si $x$ n'appartient pas à la fibre spéciale de $X_{\operatorname{sing}}\left(\right.$ resp. $\left.X_{\text {sing }}=\varnothing\right)$.

TOME $132-2004-\mathrm{N}^{\mathrm{O}} 1$ 
On dira qu'un $\mathbb{D}$-schéma formel $X$ ttf est génériquement lisse si sa fibre générique $X_{K} \rightarrow \operatorname{Spm} K$ est lisse comme $K$-espace rigide, i.e. vérifie un critère jacobien analogue à celui vérifié par les schémas lisses ( $c f .[7])$.

Remarque 2.1.10. - Si $X \rightarrow \mathbb{D}$ est un $\mathbb{D}$-schéma formel sttf lisse, il découle de la définition que $X$ est admissible, de fibre spéciale réduite et donc réduit (cf. remarque 2.1.3).

\section{Foncteur de Greenberg et (pro)-schéma associé à un schéma formel}

3.1. La construction. - Nous allons construire un foncteur

$$
\text { Gr }: \underline{\text { Form }}^{\text {sttf }} \longrightarrow \underline{\text { ProSch }} / k
$$

de la catégorie des $\mathbb{D}$-schémas formels $s t t f$ dans la catégorie des pro- $k$-schémas.

Rappelons quelques résultats de [13] et $[6, \S 9.6]$. Remarquons que, lorsque $R$ est un anneau d'égales caractéristiques et après avoir fixé un relèvement de $k$ à $R$, l'anneau $R_{n}$ peut être interprété comme l'ensemble des $k$-points d'un espace affine $\mathbf{A}_{k}^{m}$ de dimension $m$. Si $R$ est d'inégales caractéristiques, $R_{n}$ n'est plus une $k$-algèbre, mais, en utilisant les vecteurs de Witt, peut encore être considéré comme l'ensemble des $k$-points d'un $k$-schéma en anneaux, $R_{n}, k$ isomorphe, en tant que schéma, à un espace affine $\mathbf{A}_{k}^{m}(c f .[6$, p. 276]). Pour tout $n \geq 0$, considérons le foncteur $h_{n}^{*}$ qui à un $k$-schéma associe :

(i) le $k$-schéma $h_{n}^{*}(T):=T \otimes_{k} R_{n}$, en égales caractéristiques;

(ii) l'espace localement annelé $h_{n}^{*}(T)$, dont l'espace topologique sous-jacent est $T$ et le faisceau structural est $\operatorname{Hom}\left(T, R_{n}\right)$.

Soit $A$ une $k$-algèbre. On pose

$$
L(A)= \begin{cases}A, & \text { si } R \text { est un anneau d'égales caractéristiques, } \\ W(A), & \text { si } R \text { est un anneau d'inégales caractéristiques. }\end{cases}
$$

En particulier, pour toute $k$-algèbre $A$ (parfaite, si $R$ est d'inégales caractéristiques),

$$
h_{n}^{*}(\operatorname{Spec} A)=: h_{n}^{*}(A)=\operatorname{Spec}\left(R_{n} \otimes_{L(k)} L(A)\right) .
$$

REMARQUE. - L'hypothèse « $k$ parfait » est essentielle dans le cas où $R$ est un anneau d'inégales caractéristiques.

Dans [13], Greenberg a montré que, pour tout $R_{n}$-schéma $X_{n}$ localement de type fini, le foncteur

$$
T \longmapsto \operatorname{Hom}_{R_{n}}\left(h_{n}^{*}(T), X_{n}\right)
$$

de la catégorie des $k$-schémas dans celle des ensembles est représentable par un $k$-schéma $\operatorname{Gr}_{n}\left(X_{n}\right)$ qui est localement de type fini (i.e. le foncteur $h_{n}^{*}$ admet un 
adjoint à droite que l'on note $\mathrm{Gr}_{n}$ ) et vérifie, pour toute $k$-algèbre $A$ (parfaite, si $R$ est d'inégales caractéristiques),

$$
\operatorname{Gr}_{n}\left(X_{n}\right)(A) \simeq X_{n}\left(R_{n} \otimes_{L(k)} L(A)\right) .
$$

En particulier, si $A=k$, on a

$$
\operatorname{Gr}_{n}\left(X_{n}\right)(k) \simeq X_{n}\left(R_{n}\right) .
$$

Rappelons un résultat démontré par Greenberg dans [13] (cf. th., p. 643) et par Bosch, Lütkebohmert et Raynaud dans [6, p. 196 et suivantes] :

THÉORÈme 3.1.1. - Le foncteur $\mathrm{Gr}_{n}$, défini de la catégorie des $R_{n}$-schémas de type fini dans celle des $k$-schémas de type fini, vérifie les propriétés suivantes:

1) $\mathrm{Gr}_{n}$ préserve les immersions ouvertes et fermées et commute aux produits fibrés ;

2) $\mathrm{Gr}_{n}$ transforme les $R_{n}$-schémas affines en $k$-schémas affines et les $R_{n}$ schémas séparés en k-schémas séparés;

3) Si $X_{n}$ est un $R_{n}$-schéma de type fini et $\left(X_{n, i}\right)_{i \in I}$ un recouvrement fini de $X_{n}$ par des ouverts affines, alors il existe des $k$-isomorphismes de schémas canoniques $\operatorname{Gr}_{n}\left(X_{n, i} \cap X_{n, j}\right) \simeq \operatorname{Gr}_{n}\left(X_{n, i}\right) \cap \operatorname{Gr}\left(X_{n, j}\right)$ et le k-schéma $\operatorname{Gr}_{n}\left(X_{n}\right)$ s'obtient en recollant les $\operatorname{Gr}_{n}\left(X_{n, i}\right)$ le long de ces intersections.

Soit $X$ un $\mathbb{D}$-schéma formel sttf. Les morphismes canoniques $R_{n+1} \rightarrow R_{n}$, induisent alors, notamment par adjonction, pour tout $n \in \mathbf{N}$, des $k$-morphismes

$$
\theta_{n}^{n+1}: \mathrm{Gr}_{n+1}\left(X_{n+1}\right) \longrightarrow \mathrm{Gr}_{n}\left(X_{n}\right)
$$

qui font de la suite $\left(\operatorname{Gr}_{n}\left(X_{n}\right)\right)_{n \in \mathbf{N}}$ un système projectif dans la catégorie des $k$-schémas de type fini et séparés (car, pour tout $n$, le $k$-schéma $X_{n}$ est séparé par hypothèse). Enfin, comme un recouvrement (fini) de $X$ par des ouverts formels affines induit un recouvrement des $\operatorname{Gr}_{n}\left(X_{n}\right)$ (cf. théorème 3.1.1), ces morphismes de transition sont affines et leur limite $\lim _{\mathrm{Gr}_{n}}\left(X_{n}\right)$ existe dans la catégorie des $k$-schémas.

Soit $T$ un $k$-schéma. On définit $h^{*}(T)$ comme l'espace localement annelé :

(i) $\varliminf_{\lim } T \otimes_{k} R_{n}$, i.e. le complété $t$-adique du $R$-schéma $T \otimes_{k} R$, si $R$ est d'égales caractéristiques;

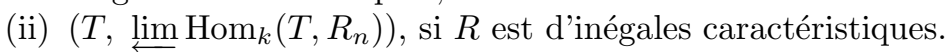

Cet espace localement annelé est muni d'un morphisme vers $\mathbb{D}$.

Proposition 3.1.2. - Soit $X$ un $\mathbb{D}$-schéma formel sttf. Le foncteur

$$
T \longmapsto \operatorname{Hom}_{\mathbb{D}}\left(h^{*}(T), X\right)
$$

de la catégorie des $k$-schémas dans celle des ensembles est représentable par un $k$-schéma canoniquement isomorphe à $\lim _{n}\left(X_{n}\right)$.

TOME $132-2004-\mathrm{N}^{\mathrm{O}} 1$ 
Démonstration. — La définition de $h^{*}(T)$ implique l'existence d'une bijection canonique :

$$
\operatorname{Hom}_{\mathbb{D}}\left(h^{*}(T), X\right) \longrightarrow \lim _{\operatorname{Hom}_{R_{n}}}\left(h_{n}^{*}(T), X_{n}\right) .
$$

L'assertion découle des propriétés universelles des $k$-schémas $\operatorname{Gr}_{n}\left(X_{n}\right)$.

Nous posons

$$
\operatorname{Gr}_{n}(X):=\left(\operatorname{Gr}_{n}\left(X_{n}\right)\right)_{\text {red }}, \operatorname{Gr}(X):=\underset{\lim }{\longleftarrow}\left(\operatorname{Gr}_{n}\left(X_{n}\right)_{\text {red }}\right) \simeq\left(\underset{\lim }{\longleftarrow} \operatorname{Gr}_{n}\left(X_{n}\right)\right)_{\text {red }} .
$$

On a donc construit un foncteur $\mathrm{Gr}$ de la catégorie des $\mathbb{D}$-schémas formels sttf dans la catégorie des pro- $k$-schémas, comme composé des foncteurs définis ci-dessus. Les définitions entraînent que, pour toute extension $F$ de $k$, les applications canoniques

$$
\operatorname{Gr}_{n}(X)(F) \longrightarrow \operatorname{Gr}_{n}\left(X_{n}\right)(F), \quad \operatorname{Gr}(X)(F) \longrightarrow\left(\lim _{\longleftarrow} \operatorname{Gr}_{n}\left(X_{n}\right)\right)(F)
$$

sont des bijections.

Exemples 3.1.3. - 1) Dans le cas d'égales caractéristiques, $R \simeq k[[\pi]]$. Soit $X$ une variété algébrique sur $k$. On peut alors considérer le $R_{n}$-schéma $X \otimes_{k} R_{n}$ pour tout $n \in \mathbf{N}$. Il découle des définitions que $\operatorname{Gr}_{n}\left(X \otimes_{k} R_{n}\right)$ est isomorphe au $k$-schéma $L_{n}(X)$ considéré dans [10]. On en déduit en particulier que $L(X) \simeq$ $\operatorname{Gr}\left(X \widehat{\otimes}_{k} R\right)$, où $X \widehat{\otimes}_{k} R$ est simplement le complété $\pi$-adique de $X \otimes_{k} R$.

2) Il découle des définitions que le $k$-schéma $\operatorname{Gr}_{0}\left(X_{0}\right)$ est canoniquement isomorphe à $X_{0}$.

3.2. Les notations. - 1) Soit $A$ une $k$-algèbre. On pose $L(A)=A$ si $R$ est un anneau d'égales caractéristiques et $L(A)=W(A)$ si $R$ est un anneau d'inégales caractéristiques. On notera par $R_{A}$ l'anneau $R_{A}:=R \widehat{\otimes}_{L(k)} L(A)$. Si $F$ est un corps contenant $k$, on notera $K_{F}$ le corps des fractions de $R_{F}$. On peut remarquer alors, que, si $k$ est supposé parfait, l'extension $R \rightarrow R_{F}$ est d'indice de ramification 1 au sens de $[6, \S 3.6]$. En particulier, on a une bijection canonique

$$
\operatorname{Gr}(X)(F) \simeq X\left(R_{F}\right) .
$$

2) Si $x \in \operatorname{Gr}(X)$ et $\kappa(x) \supset k$ son corps résiduel, on note $\varphi_{x}: \operatorname{Spf} R_{\kappa(x)} \rightarrow X$ le $\mathbb{D}$-morphisme de schémas formels correspondant à $x$ par adjonction.

3) Si $F$ est un corps contenant $k$, l'anneau $R_{F}$ n'est pas en général un anneau de valuation discrète (si $R$ est d'inégales caractéristiques). Toutefois, l'anneau $R_{F}$ s'identifie à l'ensemble $F^{\mathbf{N}}$. L'élément $y \in R_{F}$ peut donc s'identifier à la suite $\left(y_{i}\right)_{i \in \mathbf{N}}, y_{i} \in F$ pour tout $i$. Quand $F$ est parfait, $y \in R_{F}$ appartient à $(\pi)^{n}$ si et seulement si $y_{i}=0$ pour tout $1 \leq i \leq n-1$. Pour $F$ quelconque, on note $\pi^{j} y$ la suite dont les $j$ premiers termes sont nuls et telle que $\left(\pi^{j} y\right)_{i}=y_{i-j}$ pour tout $i \geq j$. Si $i \leq j$ et si $z=\pi^{j} y \in R_{F}$, on note $\pi^{-i} z:=\pi^{j-i} y$. S'il existe $y \in R_{F}$ tel que $z=\pi^{j} y$, on utilise parfois la notation $z \equiv 0 \bmod \left(\pi^{j}\right)$ ou encore $z \in \pi^{j}$. Enfin, si $x \in R_{F}^{n}$, on note $\pi^{j} x:=\left(\pi^{j} x_{i}\right)_{1 \leq i \leq n}$. 
4) Pour tout $n \in \mathbf{N}$, les morphismes canoniques seront toujours notés de la manière suivante :

$$
\operatorname{Gr}(X) \stackrel{\pi_{n, X}}{\longrightarrow} \operatorname{Gr}_{n}(X)
$$

Les morphismes $\pi_{n, X}$ (ou $\pi_{n}$ ) sont les morphismes de troncation. Les morphismes $\theta_{n-1, X}^{n}\left(\right.$ ou $\left.\theta_{n-1}^{n}\right)$ sont les morphismes de transition.

5) Soit $Y$ un $\mathbb{D}$-schéma formel sttf. Soit $h: Y \rightarrow X$ un $\mathbb{D}$-morphisme de schémas formels. On notera encore $h$ le $k$-morphisme de schémas $\operatorname{Gr}(h)$ et $h_{n}$ le $k$-morphisme $\operatorname{Gr}_{n}(h)$, pour tout $n \in \mathbf{N}$. Ces morphismes $h$ et $h_{n}$ rendent commutatif le diagramme suivant :

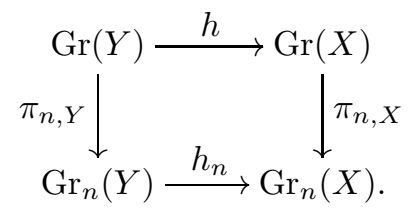

\subsection{Les propriétés et la définition par recollement}

LEMME 3.3.1. - Si $X$ est un $\mathbb{D}$-schéma formel ttf affine de fibre générique $X_{K}$, l'ensemble des points rationnels de $\operatorname{Gr}(X)$ s'identifie à un sousensemble de l'espace sous-jacent $\grave{a} X_{K}$.

Démonstration. — Ce lemme découle du lemme 3.4 de [4].

Proposition 3.3.2. - 1) Le foncteur Gr préserve les immersions ouvertes et fermées, les produits fibrés, et transforme $\mathbb{D}$-schémas formels affines en $k$ schémas affines.

2) Soit $X$ un $\mathbb{D}$-schéma formel sttf et soit $\left(O_{i}\right)_{i \in J}$ un recouvrement fini de $X$ par des sous-schémas formels ouverts affines. Il existe des isomorphismes canoniques $\operatorname{Gr}\left(O_{i} \cap O_{j}\right) \simeq \operatorname{Gr}\left(O_{i}\right) \cap \operatorname{Gr}\left(O_{j}\right)$ et le $k$-schéma $\operatorname{Gr}(X)$ est obtenu en recollant les $\operatorname{Gr}\left(O_{i}\right)$.

3) Si $X$ est un $\mathbb{D}$-schéma formel sttf, le $k$-schéma $\operatorname{Gr}(X)$ est quasi-compact.

Démonstration. - L'assertion 3) découle de 2). Les autres propiétés se déduisent directement du lemme 3.1.1.

Lemme 3.3.3. - Soit $X$ un $\mathbb{D}$-schéma formel sttf. Alors le k-morphisme de schémas canonique

$$
\operatorname{Gr}\left(X_{\text {red }}\right) \longleftrightarrow \operatorname{Gr}(X)
$$

est un isomorphisme.

TOME $132-2004-\mathrm{N}^{\mathrm{O}} 1$ 
Démonstration. - Comme $\operatorname{Gr}(X)$ et $\operatorname{Gr}\left(X_{\text {red }}\right)$ sont réduits par construction, il nous suffit de vérifier que ces deux schémas ont même espace topologique sousjacent. Soit $x \in \operatorname{Gr}(X)$. Par adjonction, il correspond à un point géométrique au-dessus de $x$, un $\mathbb{D}$-morphisme de schémas formels $\varphi: \operatorname{Spf} R_{k^{\prime}} \rightarrow X$, où $k^{\prime}$ est une clôture algébrique de $\kappa(x)$, le corps résiduel de $x$ dans $\operatorname{Gr}(X)$. Comme Spf $R_{k^{\prime}}$ est réduit, le morphisme $\varphi$ se factorise par $X_{\text {red }}$. En particulier, $x$ appartient à $\operatorname{Gr}\left(X_{\text {red }}\right)$.

Rappelons ( $c f .[24])$ la définition d'une algèbre de Tate analytiquement séparable.

DÉfinition 3.3.4. - Soit $A$ une algèbre de Tate intègre définie par un idéal

$$
\mathfrak{a} \subset K\left\{X_{1}, \ldots, X_{N}\right\} .
$$

On dit que $A$ est analytiquement séparable si l'anneau

$$
\bar{K}\left\{X_{1}, \ldots, X_{N}\right\} / \mathfrak{a} \bar{K}\left\{X_{1}, \ldots, X_{N}\right\}
$$

est réduit, où $\bar{K}$ est le complété d'une clôture algébrique de $K$. Dire que $A$ n'est pas analytiquement séparable équivaut à dire que $K$ est de caractéristique $p>0$ et que $K^{1 / p}\left\{X_{1}, \ldots, X_{N}\right\} / \mathfrak{a} K^{1 / p}\left\{X_{1}, \ldots, X_{N}\right\}$ n'est pas réduit.

Proposition 3.3.5. - Soient $X$ un $\mathbb{D}$-schéma formel admissible, réduit, dont la fibre générique est irréductible et de dimension $d$, et $X_{\operatorname{sing}}$ le sous- $\mathbb{D}$-schéma formel fermé de $X$ défini au $§ 2.1 .5$. Soient $X_{K}$ la fibre générique de $X$ et $\left(X_{\text {sing }}^{\mathrm{rig}}\right)_{K}$ celle de $X_{\text {sing }}$. Alors ou bien $\left(X_{\text {sing }}^{\text {rig }}\right)_{K}$ est de codimension au moins 1 dans $X_{K}$; ou bien, pour tout ouvert affine $U \rightarrow X$ sttf, il existe un sous- $\mathbb{D}$ schéma formel fermé $Z_{U} \hookrightarrow U$ tel que :

1) la fibre générique $\left(Z_{U}\right)_{K}$ de $Z_{U}$ est de codimension au moins 1 dans $X_{K}$;

2) le morphisme de k-schémas formels $\operatorname{Gr}\left(Z_{U}\right) \hookrightarrow \operatorname{Gr}(U)$ est bijectif.

Démonstration. - La question étant locale en $X$, on peut supposer que $X:=$ Spf $A$ est affine défini dans $R\left\{X_{1}, \ldots, X_{N}\right\}$ par un idéal $I$. Soit $I_{K}:=I \otimes_{R} K$. Par hypothèse, l'idéal $I_{K}$ est premier.

Premier cas : supposons que $A_{K}:=A \otimes_{R} K$ est analytiquement séparable. Dans [24], Schappacher a montré que le lieu singulier de $X_{K}$ est contenu strictement dans $X_{K}$. En particulier, ceci implique que la dimension de $\left(X_{\text {sing }}^{\text {rig }}\right)_{K}$ est strictement inférieure à $d\left(\operatorname{car}\left(X_{\text {sing }}^{\text {rig }}\right)_{K}\right.$ est un fermé de $X_{K}$ et $X_{K}$ est irréductible).

Deuxième cas : supposons que $A_{K}:=A \otimes_{R} K$ n'est pas analytiquement séparable. Par un argument d'analyse ultramétrique utilisé par Schappacher [24], l'hypothèse entraîne qu'il existe $g \in K\left\{X_{1}, \ldots, X_{N}\right\}$ vérifiant les propriétés suivantes : $g \notin I_{K}$ et $g(\varphi)=0$ pour tout $\varphi \in \operatorname{Gr}(X)(F)$ pour tout corps parfait $F$ contenant $k$. Quitte à multiplier par une puissance de $\pi$, on peut 
supposer en outre que $g \in R\left\{X_{1}, \ldots, X_{N}\right\}$. Le sous-D-schéma formel fermé défini par l'idéal $I+g$ est une solution au problème.

LEMme 3.3.6. - Soit $h: Y \rightarrow X$ un $\mathbb{D}$-morphisme étale de schémas formels sttf. Alors le k-schéma $\operatorname{Gr}_{n}\left(Y_{n}\right)$ est (canoniquement) isomorphe au $k$-schéma $\operatorname{Gr}_{n}\left(X_{n}\right) \times_{X_{0}} Y_{0}$, pour tout $n \in \mathbf{N}$.

Démonstration. - Dans le cas où $X$ est le complété $\pi$-adique d'une variété sur $k$, cette preuve est également faite dans [17]. On peut supposer $X$ et $Y$ affines. Soit $S=\operatorname{Spec} A$ un $k$-schéma affine. $\operatorname{Ici}_{\operatorname{Gr}_{n}}(X):=\lim _{n} \operatorname{Gr}_{n}\left(X_{n}\right)$. La donnée d'un $k$-morphisme $S \rightarrow \operatorname{Gr}_{n}\left(X_{n}\right) \times_{X_{0}} Y_{0}$ équivaut à celle d'un diagramme commutatif de $k$-morphismes :

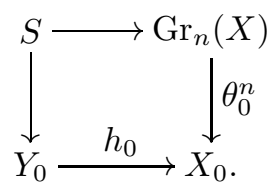

Par adjonction, celle-ci équivaut à la donnée du diagramme commutatif de $R_{n^{-}}$ morphismes

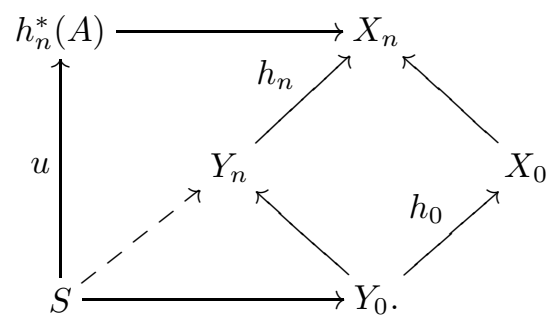

Comme le $R_{n}$-morphisme $u$ est une immersion nilpotente et que le $R_{n^{-}}$ morphisme $h_{n}$ est étale $(c f . \S 2.1 .9)$, il existe un unique $R_{n}$-morphisme $v: h_{n}^{*}(A) \rightarrow Y_{n}$, qui complète le diagramme ci-dessus en un diagramme de $R_{n}$-morphismes de schémas, où tous les triangles sont commutatifs

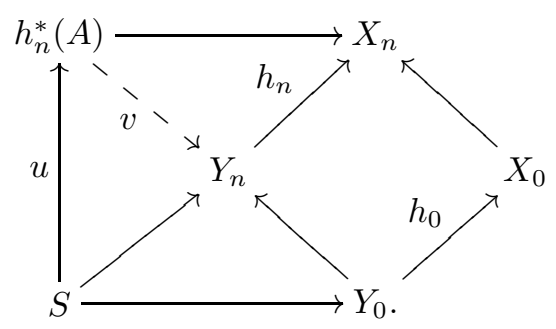

Par adjonction, on déduit l'existence d'un $k$-morphisme de schéma tel que $v$ : $S \rightarrow \mathrm{Gr}_{n}(Y)$. L'unicité de ce morphisme découle de la construction.

TOME $132-2004-\mathrm{N}^{\mathrm{O}} 1$ 
3.4. Le cas lisse. - Dans le cas où $X$ est un $\mathbb{D}$-schémas formels sttf lisse, le schéma de Greenberg associé présente quelques propriétés remarquables.

Exemple 3.4.1. - Si $X=\mathbb{B}_{R}^{d}$, le $k$-schéma $\operatorname{Gr}_{n}(X)$ est $k$-isomorphe à $\mathbf{A}_{k}^{(n+1) d}$.

Lemme 3.4.2. - Soit $X \rightarrow \mathbb{D}$ un $\mathbb{D}$-schéma formel sttf, lisse, de pure dimension relative $d$. Alors on a les propriétés suivantes :

1) pour tout $n \in \mathbf{N}$ et tout $m \geq n$, les $k$-morphismes $\pi_{n, X}$ et $\theta_{n}^{m}$ sont surjectifs ;

2) pour tout $n \in \mathbf{N}$, le $k$-morphisme $\theta_{n}^{n+1}$ est une fibration localement triviale de fibre $\mathbf{A}_{k}^{d}$ pour la topologie de Zariski.

Démonstration. — L'assertion 1) peut se voir directement comme une conséquence du lemme de Hensel (cf. [9, chap. III, $\S 4, \mathrm{n}^{\circ} 6$, cor. 2]) ou comme une conséquence de 2). Comme $X$ est lisse, pour tout $x \in X_{0}$, il existe un sous$\mathbb{D}$-schéma formel affine $U \hookrightarrow X$, ouvert contenant $x$, et un $\mathbb{D}$-morphisme de schémas formels étale $U \rightarrow \mathbb{B}_{R}^{d}$. Le lemme 3.3.6 et $\operatorname{Gr}_{n}\left(\mathbb{B}_{R}^{d}\right)=\mathbf{A}_{k}^{(n+1) d}$ assurent le résultat.

\section{Cylindres et leur mesure}

4.1. Les anneaux de Grothendieck. - Soit $k$ un corps. On note $K_{0}(\underline{\operatorname{Var}} / k)$ le groupe abélien engendré par les symboles $[S]$, pour $S$ une variété sur $k$ (i.e. un $k$-schéma de type fini réduit et séparé), avec les relations $[S]=\left[S^{\prime}\right]$ si $S$ et $S^{\prime}$ sont isomorphes et $[S]=\left[S^{\prime}\right]+\left[S \backslash S^{\prime}\right]$ si $S^{\prime}$ est une sous-variété fermée de $S$. Ce groupe possède une structure naturelle d'anneau, dont le produit est induit par le produit fibré. La classe de Spec $k$ est l'élément neutre de cet anneau; on la note 1. En référence au motif de Lefschetz, on note également $\mathbf{L}$ la classe de la droite affine dans $K_{0}(\underline{\operatorname{Var}} / k)$.

Remarque 4.1.1. - Comme l'a prouvé Poonen dans [22], cet anneau n'est pas intègre. Par ailleurs la définition de $K_{0}\left(\underline{\operatorname{Var}}_{/ k}\right)$ explique pourquoi l'on a muni $\operatorname{Gr}_{n}\left(X_{n}\right)$ de sa structure réduite.

Soit $S$ une $k$-variété. Tout sous-ensemble constructible $C$ de $S$ peut s'écrire comme réunion finie disjointe de sous- $k$-variétés de $S$, i.e. il existe des sous- $k$ variétés $\left(S_{i}\right)_{1 \leq i \leq n}$ de $S$ telles que

$$
C=\bigsqcup_{1 \leq i \leq n} S_{i}
$$

À tout ensemble constructible $C$ de $S$, on peut donc associer naturellement une unique classe $[C]$ dans $K_{0}\left(\underline{\operatorname{Var}}_{k}\right)$ de sorte que, si $C$ et $C^{\prime}$ sont deux ensembles constructibles d'une variété $S$, on a la relation $\left[C \cup C^{\prime}\right]=[C]+\left[C^{\prime}\right]-\left[C \cap C^{\prime}\right]$. 
On désigne par

$$
M_{k}:=K_{0}\left(\underline{\operatorname{Var}}_{k}\right)\left[\mathbf{L}^{-1}\right]
$$

le localisé de $K_{0}\left(\underline{\operatorname{Var}}_{/ k}\right)$ par rapport au système multiplicatif $\left\{\mathbf{1}, \mathbf{L}, \mathbf{L}^{2}, \ldots\right\}$. Soient $F^{m} M_{k}$ le sous-groupe de $M_{k}$ engendré par les $[S] \mathbf{L}^{-i}$ tels que $\operatorname{dim} S-i \leq-m$, et $\widehat{M}_{k}$ le séparé complété de $M_{k}$ suivant cette filtration. On note $\bar{M}_{k}$ l'image de $M_{k}$ dans $\widehat{M}_{k}$.

La filtration $F^{\bullet}$ définit une topologie métrisable sur $\widehat{M}_{k}$. L'application

$$
\|\|: \widehat{M}_{k} \longrightarrow \mathbf{R}_{\geq 0}
$$

définie par

$$
\|a\|= \begin{cases}2^{-n} & \text { si } a \in F^{n} \widehat{M}_{k} \text { et } a \notin F^{n+1} \widehat{M}_{k}, \\ 0 & a=0,\end{cases}
$$

est la norme induite par cette filtration. Elle munit $\widehat{M}_{k}$ d'une structure d'anneau normé non archimédien, séparé et complet.

4.2. Les fibrations par morceaux. - Nous allons définir la notion de fibration localement triviale par morceaux, qui correspond à l'idée de fibration localement triviale pour la topologie constructible (cf. [16, 7.2.11] pour une définition de la topologie constructible).

DÉfinition 4.2.1. - Soient $X, Y$ et $F$ trois $k$-schémas, $A$ (resp. $B$ ) une partie constructible de $X$ (resp. $Y$ ). Supposons que $F$ est de type fini.

1) On dit que l'application $\pi: B \rightarrow A$ est une fibration triviale par morceaux de fibre $F$, s'il existe une partition finie de $A$ par des sous-ensembles $S$ localement fermés dans $X$ tel que la restriction de $\pi$ à $\pi^{-1}(S)$ soit induite par un morphisme de $k$-schémas $Y \rightarrow X$ et tel que $\pi^{-1}(S)$, qui est localement fermé dans $Y$, soit isomorphe en tant que $k$-schéma, à $S \times_{k} F, \pi$ correspondant, par cet isomorphisme à la projection $S \times{ }_{k} F \rightarrow S$.

2) On dit que l'application $\pi$ est une fibration triviale par morceaux sur $C$, avec $C$ un sous-ensemble constructible de $B$, si la restriction de $\pi$ à $\pi^{-1}(C)$, i.e. $\pi_{\mid \pi^{-1}(C)}: \pi^{-1}(C) \rightarrow C$, est une fibration triviale par morceaux.

Lemme 4.2.2. - Soient $X$ et $Y$ deux k-variétés. Soient $A$ (resp. B) une partie constructible de $X$ (resp. $Y$ ). Si $\pi: B \rightarrow A$ est une fibration triviale par morceaux de fibre $F$, on a dans $M_{k}$ la formule

$$
[B]=[A] \cdot[F]
$$

Démonstration. - Ceci découle de la formule d'additivité de [] dans $M_{k}$ (cf. $\S 4.1)$.

TOME $132-2004-\mathrm{N}^{\mathrm{O}} 1$ 
ThÉorÈme 4.2.3. - Soient $X$ et $Y$ deux $k$-variétés, et $F$ un $k$-schéma de type fini. Soient $A$ et $B$ deux ensembles constructibles de $X$ et $Y$ respectivement. Soit $h: B \rightarrow A$ une application induite par un $k$-morphisme de schémas $Y \rightarrow X$. Alors cette application $h$ est une fibration localement triviale par morceaux de fibre $F$ si et seulement si, pour tout $x \in B$, la fibre $\pi^{-1}(x)$ est un $\kappa(x)$-schéma isomorphe à $F_{\kappa(x)}:=F \otimes_{k} \kappa(x)$.

Démonstration. - Il est clair que, si $h$ est une fibration localement triviale par morceaux, alors, pour tout $x \in B$, la fibre $\pi^{-1}(x)$ est $\kappa(x)$-isomorphe à $F_{\kappa(x)}$. Réciproquement, supposons que $h$ vérifie cette dernière hypothèse. Il nous faut donc prouver l'existence d'une partition finie de $B$ en sous- $k$-variétés localement fermées, au-dessus desquelles $h$ s'identifie à une projection. Par définition, il existe une partition finie $\left(S_{i}\right)_{i \in I}$ de $A$ en sous- $k$-variétés localement fermées de $X$. Il est clair qu'il nous suffit de prouver l'existence d'une telle partition sur chaque $S_{i}, i \in I$. On peut donc supposer que $B=h^{-1}(S), A=S$ et $S \hookrightarrow X$ une sous- $k$-variété localement fermée de $X$. Le lemme 4.2 .4 ci-dessous assure l'existence d'un ouvert $U_{0} \hookrightarrow S$, dense dans $S$, tel que la restriction de $h$ à $h^{-1}\left(U_{0}\right)$ s'identifie au $k$-morphisme canonique $U_{0} \times_{k} F \rightarrow U_{0}$. Posons $Z_{0}:=S \backslash U_{0}$. Par définition, $Z_{0} \hookrightarrow S$ est une sous- $k$-variété fermée de $S$ et localement fermée dans $X$. En appliquant à nouveau le lemme 4.2 .4 à $Z_{0}$, on déduit l'existence d'un ouvert $U_{1}$ de $Z_{0}$ dense et de complémentaire $Z_{1}$ (dans $Z_{0}$ ). Par suite, il existe, en réitérant ce raisonnement, une suite décroissante de parties fermées dans $S$

$$
\cdots \subset Z_{n} \subset Z_{n-1} \subset \cdots \subset Z_{0} \subset S
$$

tels que $Z_{i}$ est d'intérieur vide dans $Z_{i-1}$. Comme $S$ est noethérien, il existe un entier $N \in \mathbf{N}$ tel que $Z_{N}$ est vide. Ceci signifie que

$$
S=\bigsqcup_{0 \leq j \leq N} U_{j} .
$$

Par ailleurs, par construction, $h$ s'identifie au-dessus de chaque $U_{j}$, qui est localement fermé dans $X$, à la projection $U_{j} \times_{k} F \rightarrow U_{j}$.

Lemme 4.2.4. - Soient $V, W$ deux $k$-schémas de type fini et $F$ un $k$-schéma de type fini. Soit $p: W \rightarrow V$ un $k$-morphisme de schémas tel que, pour tout $x \in V$, le $\kappa(x)$-schéma $p^{-1}(x)$ est $\kappa(x)$-isomorphe à $F_{\kappa(x)}$. Alors il existe un ouvert dense $U$ de $V$ et un k-isomorphisme $\tilde{p}: p^{-1}(U) \rightarrow U \times_{k} F$ tel que le diagramme suivant soit commutatif:

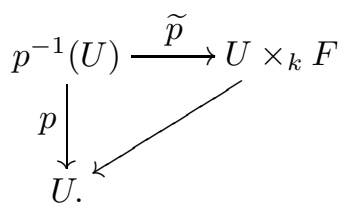


Démonstration. - La propriété étant locale sur la base, on peut supposer que $V:=\operatorname{Spec} A$, où $A$ est une $k$-algèbre de type fini. On peut supposer en outre que $V$ est irréductible. Ce lemme est alors une conséquence directe du théorème 8.8.2 de [15] et de son corollaire 8.8.2.5.

4.3. Les cylindres. — Fixons un $\mathbb{D}$-schéma formel sttf $X$ de dimension relative $d$.

DÉFInition 4.3.1. - On dit qu'une partie $A$ de $\operatorname{Gr}(X)$ est un sous-ensemble cylindrique de rang $n$ de $\operatorname{Gr}(X)$ (ou plus simplement un $n$-cylindre de $\operatorname{Gr}(X)$ ), si $A=\pi_{n, X}^{-1}(C)$, où $C$ désigne une partie constructible de $\operatorname{Gr}_{n}(X)$. On dit que $A$ est un cylindre si $A$ est un cylindre d'un certain rang $n$.

On dit qu'une partie $A$ de $\operatorname{Gr}(X)$ est un pro-cylindre (resp. un ind-cylindre) si elle est intersection (resp. réunion) de cylindres.

Lemme 4.3.2. - Soit $A$ un cylindre de rang $n$ de $\operatorname{Gr}(X)$. Alors

$$
A=\pi_{n, X}^{-1}\left(\pi_{n, X}(A)\right) .
$$

Démonstration. - Cet énoncé se déduit facilement du fait que, par définition, $A:=\pi_{n, X}^{-1}\left(A_{n}\right)$ avec $A_{n}$ ensemble constructible de $\operatorname{Gr}_{n}(X)$.

Exemples 4.3.3. - 1) L'espace $\operatorname{Gr}(X)$ est un 0-cylindre, puisque $\pi_{0, X}^{-1}\left(X_{0}\right)=$ $\operatorname{Gr}(X)$.

2) Soit $U \hookrightarrow X$ un sous- $\mathbb{D}$-schéma formel ouvert de $X$ quasi-compact. Le $k$-schéma $\operatorname{Gr}(U) \hookrightarrow \operatorname{Gr}(X)$ est un 0-cylindre de $\operatorname{Gr}(X)$ de rang 0. Plus précisément, on a l'égalité

$$
\operatorname{Gr}(U)=\pi_{0, X}^{-1}\left(\operatorname{Gr}_{0}(U)\right) .
$$

3) Soit $Z \hookrightarrow X$ un sous-D-Dchéma fermé de $X$. Alors $\operatorname{Gr}(Z)$ est un procylindre. En effet, on a l'égalité

$$
\operatorname{Gr}(Z)=\bigcap_{n \in \mathbf{N}} \pi_{n, X}^{-1}\left(\operatorname{Gr}_{n}(Z)\right)
$$

Proposition 4.3.4. - Tout cylindre est un sous-ensemble constructible de $\operatorname{Gr}(X)$.

Démonstration. - Ce lemme découle du fait que l'image inverse d'un constructible est constructible ( $c f .[16, \S 7$, prop. 7.1.2]).

LEMme 4.3.5. - Si A est un cylindre de rang $n$, alors A est aussi un cylindre de rang $m$, pour tout $m \geq n$.

TOME $132-2004-\mathrm{N}^{\mathrm{O}} 1$ 
Démonstration. — Ce lemme découle de la commutativité du diagramme

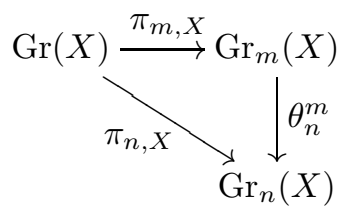

et $d u$ fait que l'image inverse d'un constructible est constructible ( $c f .[16, \S 7$, prop. 7.1 .2$]$ ).

On note $\mathbf{C}_{X}$ l'ensemble des cylindres de $\operatorname{Gr}(X)$.

Proposition 4.3.6. - L'ensemble $\mathbf{C}_{X}$ des cylindres de $\operatorname{Gr}(X)$ est un anneau booléen. Autrement dit :

1) les ensembles $\operatorname{Gr}(X)$ et $\varnothing$ appartiennent à $\mathbf{C}_{X}$;

2) l'ensemble $\mathbf{C}_{\mathbf{X}}$ est stable par intersection finie;

3) l'ensemble $\mathbf{C}_{X}$ est stable par réunion finie;

4) l'ensemble $\mathbf{C}_{\mathbf{X}}$ est stable par passage au complémentaire.

Démonstration. - Grâce au lemme 4.3.5, on se ramène au cas de cylindres de même rang. Cette proposition découle alors du fait que les parties constructibles d'un ensemble vérifient ces propriétés et de la stabilité de ces propriétés pour l'image inverse ( $c f .[16, \S 7$, prop. 7.1 .2$]$ ).

LEMmE 4.3.7. - Soit $\left(A_{i}\right)_{i \in I}$ une famille de cylindres de $\operatorname{Gr}(X)$. Si $A:=$ $\bigcup_{i \in I} A_{i}$ est également un cylindre, alors il existe un sous-ensemble $J$ fini de $I$ tel que $A=\bigcup_{i \in J} A_{i}$. Plus généralement, si $A$ est un pro-cylindre contenu dans la réunion des cylindres $A_{i}, i \in I$, il existe un sous-ensemble $J$ fini de $I$ tel que $A \subset \bigcup_{i \in J} A_{i}$.

Démonstration. - En remarquant que $\operatorname{Gr}(X)$ est quasi-compact (obtenu par recollement d'un nombre fini d'ouverts affines), ce lemme est une conséquence de la quasi-compacité de la topologie constructible ( $c f .[16, \S 7$, prop. 7.2.13]).

Le résultat suivant, qui est une reformulation d'un théorème de Schappacher [24], est l'analogue du théorème de Greenberg [14] dans le cadre de la théorie des schémas formels ( $c f$. loc. cit. pour les énoncés originaux).

ThÉORÈme 4.3.8. - Soit $R$ un anneau de valuation discrète complet et $X$ un $\mathbb{D}$-schéma formel admissible. Pour tout $n \geq 0$, il existe un entier $m_{X}(n) \geq n$, ne dépendant que de $X$, tel que, pour tout corps parfait $F$ contenant $k$, et tout $x \in X\left(R_{F} /(\pi)^{m_{X}(n)}\right)$, l'image de $x$ dans $X\left(R_{F} /(\pi)^{n}\right)$ peut être relevée 
en $\widetilde{x} \in X\left(R_{F}\right)$. On appelle fonction de Greenberg du schéma formel $X$ l'application qui à $n \in \mathbf{N}$ associe le plus petit des entiers $m_{X}(n)$ définis ci-dessus. On notera $\gamma_{X}$ cette application.

Ce théorème permet de démontrer le lemme suivant, qui joue un rôle crucial dans la définition de la mesure motivique au niveau des cylindres.

LEMme 4.3.9. - L'image $\pi_{n, X}(\mathrm{Gr}(X))$ de $\operatorname{Gr}(X)$ dans $\mathrm{Gr}_{n}(X)$ est un ensemble constructible de $\operatorname{Gr}_{n}\left(X_{n}\right)$. Plus généralement, si A est un cylindre de rang $m$ de $G(X)$, alors $\pi_{n, X}(A)$ est un ensemble constructible de $\operatorname{Gr}_{n}(X)$, pour tout $n \geq 0$.

Démonstration. - Il nous suffit de démontrer la seconde assertion. Par définition, il existe un ensemble constructible $C_{m}$ de $\operatorname{Gr}_{m}(X)$ tel que $A=\pi_{m}^{-1}\left(C_{m}\right)$ (on omettra l'indice $X$ dans $\pi_{n, X}$ ). On peut supposer que $m=n$. En effet, si $n \geq m$, le lemme 4.3 .5 assure que $A$ est un $n$-cylindre; si $m \geq n$, la relation $\pi_{n}(A)=\theta_{n}^{m}\left(\pi_{m}\left(A_{m}\right)\right)$ et un théorème de Chevalley ( $c f .[16, \S 7$, th. 7.1.4]) entraîne que, si $\pi_{m}(A)$ est constructible, $\pi_{n}(A)$ l'est aussi. Le théorème de Greenberg 4.3.8 implique que

$$
\pi_{n}(A)=\theta_{n}^{\gamma_{X}(n)}\left(\left(\theta_{n}^{\gamma_{X}(n)}\right)^{-1}\left(C_{n}\right)\right) .
$$

Le théorème de Chevalley déjà cité permet alors de conclure.

DÉfinition 4.3.10. — Si $d$ est la dimension relative de $X$, on dit qu'un sousensemble $A$ de $\operatorname{Gr}(X)$ est un cylindre stable de rang $n$ si :

1) l'ensemble $A$ est un cylindre de rang $n$;

2 ) pour tout $m \geq n$, la restriction du morphisme de transition

$$
\pi_{m+1, X}(\operatorname{Gr}(X)) \longrightarrow \pi_{m, X}(\operatorname{Gr}(X))
$$

est une fibration triviale par morceaux de fibre $\mathbf{A}_{k}^{d}$ sur $\pi_{m, X}(A)$.

Exemple 4.3.11. - Soit $e \in \mathbf{N}$. Un exemple important de cylindre est le sous-ensemble $\operatorname{Gr}^{(e)}(X)$ de $\operatorname{Gr}(X)$ défini par

$$
\operatorname{Gr}^{(e)}(X):=\operatorname{Gr}(X) \backslash\left(\pi_{e, X}^{-1}\left(\operatorname{Gr}_{e}\left(X_{\text {sing }}\right)\right)\right) .
$$

On en déduit que

$$
\operatorname{Gr}(X)=\left(\bigcup_{e \in \mathbf{N}} \operatorname{Gr}^{(e)}(X)\right) \sqcup \operatorname{Gr}\left(X_{\text {sing }}\right) .
$$

En fait, nous allons montrer ( $c f$. lemme 4.5.4) que ce cylindre est $e$-stable.

Soit $\mathbf{C}_{0, X}$ la famille des sous-ensembles cylindriques de $\operatorname{Gr}(X)$ qui sont stables pour un certain rang $n$.

TOME $132-2004-\mathrm{N}^{\mathrm{O}} 1$ 
Proposition 4.3.12. - L'ensemble $\mathbf{C}_{0, X}$ des cylindres stables de $\operatorname{Gr}(X)$ est un idéal de $\mathbf{C}_{\mathbf{X}}$. Autrement dit, il vérifie les propriétés suivantes :

1) L'ensemble $\mathbf{C}_{0, X}$ est contenu dans $\mathbf{C}_{X}$ et contient $\varnothing$.

2) Si $A, B \in \mathbf{C}_{0, X}$ sont deux cylindres stables disjoints, alors leur réunion est un cylindre stable.

3) Si $A \in \mathbf{C}_{0, X}$ et $B \in \mathbf{C}_{X}$, alors $A \cap B$ est un cylindre stable.

Démonstration. - Ces propriétés découlent de la proposition 4.3.6 et de la définition des cylindres stables.

L'intérêt des cylindres stables est que l'on peut définir une mesure « naïve » sur cet ensemble de la manière suivante.

Proposition 4.3.13. - 1) Il existe une unique application additive

$$
\mu_{0, X}: \mathbf{C}_{0, X} \longrightarrow M_{k}
$$

tel que $\mu_{0, X}(A)=\left[\pi_{n, X}(A)\right] \mathbf{L}^{-(n+1) d}$, pour tout cylindre A stable au rang $n$.

2) L'application $A \mapsto \mu_{0, X}(A)$ est $\sigma$-additive sur $\mathbf{C}_{0, X}$.

3) Pour $A$ et $B$ dans $\mathbf{C}_{0, X}$, on a

$$
\left\|\mu_{0, X}(A \cup B)\right\| \leq \max \left(\left\|\mu_{0, X}(A)\right\|,\left\|\mu_{0, X}(B)\right\|\right) .
$$

Si $A \subset B$, on a $\left\|\mu_{0, X}(A)\right\| \leq\left\|\mu_{0, X}(B)\right\|$.

Démonstration. - Soit $A$ un cylindre stable de rang $n$ de $\operatorname{Gr}(X)$. Comme, par définition, l'application $\theta_{n}^{m}$ est sur $\pi_{n}(A)$ une fibration localement triviale par morceaux de fibre $\mathbf{A}_{k}^{d}$ pour tout $m \geq n$, on a dans $M_{k}$ la relation

$$
\left[\pi_{m, X}(A)\right]=\left[\pi_{n, X}(A)\right] \mathbf{L}^{(m-n) d} .
$$

Cette relation montre que l'application $\mu_{0, X}$ est bien définie. L'additivité de l'application [ ] entraîne l'additivité de $\mu_{0, X}$. Pour la $\sigma$-additivité, on se ramène au cas de l'additivité grâce au lemme 4.3.7. La dernière assertion découle de la définition de la norme.

4.4. Une estimation de la dimension des fibres des morphismes de transition. - Les lemmes précédents permettent de borner la dimension des fibres des morphismes $\theta_{n, X}^{m}$.

LEMME 4.4.1. - Soit $X$ un $\mathbb{D}$-schéma formel sttf, dont la fibre générique $X_{K}$ est de dimension d. Alors :

1) pour tout $n \in \mathbf{N}$, $\operatorname{dim} \pi_{n, X}(\operatorname{Gr}(X)) \leq(n+1) d$;

2) pour tout $n, m \in \mathbf{N}$, tel que $m \geq n$, les fibres de

$$
\pi_{m, X}(\operatorname{Gr}(X)) \longrightarrow \pi_{n, X}(\operatorname{Gr}(X))
$$

sont de dimension inférieure à $(m-n) d$. 
Démonstration. — Supposons connue l'assertion 4.4.1, 2). Appliquons-la aux entiers $n \geq 0$ et 0 . On a donc

$$
\operatorname{dim} \pi_{n}(\operatorname{Gr}(X)) \leq n d+\operatorname{dim} \pi_{0}(\operatorname{Gr}(X)) \leq(n+1) d .
$$

Pour montrer l'assertion 4.4.1,2), il nous suffit de prouver que, pour tout $n \in \mathbf{N}$, la dimension de chaque fibre du morphisme $\pi_{n+1}(\operatorname{Gr}(X)) \rightarrow \pi_{n}(\operatorname{Gr}(X))$ est inférieure ou égale à $d$. La stratégie est alors d'identifier ces fibres à des sousschémas d'un schéma de dimension $d$.

On peut supposer que $X$ est affine de la forme $\operatorname{Spf} R\left\{x_{1}, \ldots, x_{N}\right\} /\left(f_{1}, \ldots, f_{m}\right)$. Posons $f:=\left(f_{1}, \ldots, f_{m}\right)$. Soit $x \in \operatorname{Gr}(X)$. Soit $\widetilde{\xi} \in \operatorname{Gr}(X)\left(\kappa(x)^{\text {alg }}\right)$ le $k$-morphisme qui correspond à un point géométrique au-dessus de $x$ et $\xi \in\left(R_{\kappa(x)^{\text {alg }}}\right)^{N}$ le $N$-uplet de $\left(R_{\kappa(x)^{\text {alg }}}\right)^{N}$ qui correspond à ce morphisme. On peut supposer que $k=\kappa(x)^{\text {alg }}$ et que $\varphi \in R^{N}$. Soit $Y$ le $R$-schéma formel affine $t t f$ défini par le système de séries formelles restreintes

$$
g(y)=f\left(\xi+\pi^{n+1} y\right), \quad \text { avec } \quad y:=\left(y_{1}, \ldots, y_{N}\right) \in \operatorname{Spf} R\{y\} .
$$

Le $K$-morphisme d'algèbres topologiques qui à $x_{i}$ associe $\xi_{i}+\pi^{n+1} y_{i}$ pour tout $1 \leq i \leq N$ induit un isomorphisme d'espaces rigides entre $X_{\text {rig }}$ et $Y_{\text {rig. }}$. En particulier, $Y_{\text {rig }}$ est de dimension $d$. Il existe un $R$-modèle $Y^{\prime}$ de $Y_{\text {rig }}$ défini, dans $\mathbb{B}_{R}^{N}$, par le système d'équations $g(y) / \pi^{n+1}$. Tout point de la fibre est alors contenu dans la fibre spéciale $Y_{0}^{\prime}$ de $Y^{\prime}$. Le théorème 1 établi par Oesterlé [21] (cf. théorème 2.1.7) assure alors que la fibre est de dimension au plus $d$.

Signalons cette conséquence du lemme 4.4.1.

LEMme 4.4.2. - Soit $X$ un $\mathbb{D}$-schéma formel admissible, dont la fibre générique $X_{K}$ est de dimension d et soit $S$ un sous-D-Dchéma formel fermé, dont la fibre générique $S_{K}$ est de dimension strictement inférieure à d. Soit $\gamma_{S}$ la fonction de Greenberg pour $S$. Alors pour tous $n, i, e \in \mathbf{N}$, tels que $n \geq i \geq \gamma_{S}(e)$, $\pi_{n, X}\left(\pi_{i, X}^{-1} \operatorname{Gr}_{e}(S)\right)$ est de dimension inférieure à $(n+1) d-e-1$. Autrement dit, pour tous $n \geq i \geq \gamma_{S}(e)$,

$$
\left[\pi_{n, X}\left(\pi_{i, X}^{-1}\left(\operatorname{Gr}_{i}(S)\right)\right)\right] \cdot \mathbf{L}^{-(n+1) d} \in F^{e+1} M_{k} .
$$

Démonstration. - On peut supposer que $i=\gamma_{S}(e)$. Par le lemme 4.4.1, 2) appliqué à la projection

$$
\pi_{n, X}\left(\pi_{\gamma_{S}(e), X}^{-1}\left(\operatorname{Gr}_{\gamma_{S}(e)}(S)\right)\right) \longrightarrow \pi_{e, X}\left(\pi_{\gamma_{S}(e), X}^{-1}\left(\operatorname{Gr}_{\gamma_{S}(e)}(S)\right)\right)
$$

on obtient l'inégalité

$$
\operatorname{dim} \pi_{n X}\left(\pi_{\gamma_{S}(e), X}^{-1}\left(\operatorname{Gr}_{\gamma_{S}(e)}(S)\right)\right) \leq(n-e) d+\operatorname{dim} \pi_{e, X}\left(\pi_{\gamma_{S}(e), X}^{-1}\left(\operatorname{Gr}_{\gamma_{S}(e)}(S)\right)\right)
$$

Par ailleurs, par définition de la fonction de Greenberg,

$$
\pi_{e, X}\left(\pi_{\gamma_{S}(e), X}^{-1}\left(\operatorname{Gr}_{\gamma_{S}(e)}(S)\right)\right)=\pi_{e, X}(\operatorname{Gr}(S)) .
$$

TOME $132-2004-\mathrm{N}^{\mathrm{O}} 1$ 
Le lemme 4.4.1, 1), assure que $\operatorname{dim} \pi_{e, X}(\operatorname{Gr}(S)) \leq(e+1)(d-1)$. Le résultat découle du fait que $(n-e) d+(e+1)(d-1)=(n+1) d-e-1$.

Corollaire 4.4.3. - Soit $X$ un $\mathbb{D}$-schéma formel admissible. Soit $B$ un cylindre de $\operatorname{Gr}(X)$ de rang $m$ tel qu'il existe un sous- $\mathbb{D}$-schéma formel $Z$ de $X$ génériquement de codimension 1, dont le k-schéma de Greenberg associé contient B. Alors, pour tout $n \geq \max \left(m, \gamma_{Z}(e)\right)$,

$$
\left[\pi_{n, X}(B)\right] \cdot \mathbf{L}^{-(n+1) d} \in F^{e+1} M_{k}
$$

4.5. La mesure pour les cylindres. - Soit $e \in \mathbf{N}$. Posons

$$
\operatorname{Gr}^{(e)}(X):=\operatorname{Gr}(X) \backslash \pi_{e, X}^{-1}\left(\operatorname{Gr}_{e}\left(X_{\text {sing }}\right)\right) .
$$

Cet ensemble est un e-cylindre par définition. Rappelons un résultat d'algèbre commutative :

Lemme 4.5.1. - Soit $X:=\operatorname{Spf} A$ un $\mathbb{D}$-schéma formel affine admissible, purement de dimension relative $d$. Si $A:=R\left\{x_{1}, \ldots, x_{N}\right\} / I$, alors $X_{\text {sing }}$ est le sous-D-schéma formel de $X$ défini par le radical dans $A$ de l'idéal

$$
\sum \Delta\left(f_{1}, \ldots, f_{N-d}\right)\left(\left(f_{1}, \ldots, f_{N-d}\right): I\right)
$$

La somme est prise sur l'ensemble des $(N-d)$-uplets $\left(f_{1}, \ldots, f_{N-d}\right) \in I$ et la notation $\Delta\left(f_{1}, \ldots, f_{N-d}\right)$ désigne l'idéal engendré par les mineurs d'ordre $N-d$ de la matrice jacobienne

$$
\left(\frac{\partial f_{j}}{\partial x_{i}}\right)_{1 \leq j \leq N-d, 1 \leq i \leq N}
$$

Démonstration. — La preuve du théorème 4.1 de [26] s'adapte sans difficulté.

Les lemmes 4.5.2 et 4.5.3 vont intervenir dans la démonstration du lemme clé 4.5.4.

Lemme 4.5.2. - Soit $X:=\operatorname{Spf} R\left\{X_{1}, \ldots, X_{N}\right\} / I \hookrightarrow \mathbb{B}_{R}^{N}$ un $\mathbb{D}$-schéma formel admissible, purement de dimension relative $d$. Alors il existe un entier $c_{X} \in \mathbf{N} \backslash\{0\}$ tel que pour tout $x \in \operatorname{Gr}(X) \backslash \pi_{e, X}^{-1}\left(\mathrm{Gr}_{e}\left(X_{\text {sing }}\right)\right)$, on peut trouver des séries formelles restreintes $f_{1}, \ldots, f_{N-d}$ appartenant à l'idéal $I, h$ appartenant au conducteur de I dans $\left(f_{1}, \ldots, f_{N-d}\right)$ et $\delta$ appartenant à l'ensemble des mineurs d'ordre $(N-d)$ de la matrice jacobienne $\left(\partial f_{i} / \partial x_{j}\right)_{1 \leq i \leq N-d, 1 \leq j \leq N}$ de sorte que, si $Z \hookrightarrow X$ est le sous-D-schéma formel fermé de $\bar{X}$ défini par l'idéal $I+(h \delta)$, on a

$$
x \notin \pi_{c_{X} e+1, X}^{-1}\left(\operatorname{Gr}_{c_{X} e+1}(Z)\right)
$$

BULletin DE LA SOCiÉtÉ MATHÉMATIQUE DE FRANCE 
Démonstration. - Si $J$ est l'idéal engendré par les éléments

$$
g \in \sum \Delta\left(f_{1}, \ldots, f_{N-d}\right)\left(\left(f_{1}, \ldots, f_{N-d}\right): I\right),
$$

la somme étant prise sur les $(N-d)$-uplets $\left(f_{i_{1}}, \ldots, f_{i_{N-d}}\right) \in I$, alors le lemme 4.5 .1 assure que $X_{\text {sing }}$ est défini par l'idéal $\sqrt{I+J}$. Soit $c_{X}$ le plus petit des entiers naturels $c$ tels que $(\sqrt{I+J})^{c} \subset I+J$.

Soient $x \in \mathrm{Gr}^{(e)}(X)$ de corps résiduel $k^{\prime}:=\kappa(x) \supset k$ et $\varphi: \operatorname{Spf} R_{k^{\prime}} \rightarrow X$ le $\mathbb{D}$-morphisme de schémas formels correspondant à $x$. Par hypothèse, il existe $g \in \sqrt{I+J}$ tel que

$$
g(\varphi) \not \equiv 0 \quad \bmod \left(\pi^{e+1}\right)
$$

En particulier, ceci implique l'existence d'un élément $u \in J$ tel que

$$
u(\varphi) \not \equiv 0 \quad \bmod \left(\pi^{c_{X} e+1}\right) .
$$

L'idéal $J$ étant engendré par les éléments $h \delta$, avec $h$ appartenant au conducteur de $I$ dans $\left(f_{i_{1}}, \ldots, f_{i_{N-d}}\right)$ et $\delta$ un mineur d'ordre $N-d$ de la matrice jacobienne de ce système, on peut supposer que $u=h \delta$. Le résultat cherché est alors une simple retraduction de la dernière congruence.

LEMme 4.5.3. - Soit $X$ un $\mathbb{D}$-schéma formel affine admissible et purement de dimension $d$, d'algèbre $A:=R\left\{X_{1}, \ldots, X_{N}\right\} / I$. Soit $\Lambda_{N-d}$ l'ensemble des $(N-d)$-uplets $\left(f_{1}, \ldots, f_{N-d}\right) \in I . \grave{A} f \in \Lambda_{N-d}$ on associe le $\mathbb{D}$-schéma formel sttf affine $Z(f)$ défini dans $\mathbb{B}_{R}^{N}$ par $f_{i}=0$, pour tout $1 \leq i \leq N-d$; et, pour toute injection $\sigma:\{1, \ldots, N-d\} \rightarrow\{1, \ldots, N\}$, on note $\delta_{f, \sigma}$ le déterminant de la matrice

$$
\left(\frac{\partial f_{i}}{\partial x_{\sigma(j)}}\right)_{1 \leq i \leq N-d, 1 \leq j \leq N-d} .
$$

Alors, pour tout $e \in \mathbf{N}$, il existe un recouvrement fini de $\operatorname{Gr}^{(e)}(X)$ par des $c_{X}$ e-cylindres $B_{i}, i \in I$, de $\operatorname{Gr}\left(\mathbb{B}_{R}^{N}\right)$ vérifiant les propriétés suivantes :

1) pour tout $i \in I$, il existe $f_{i} \in \Lambda_{N-d}$ tel que

$$
B_{i} \cap \operatorname{Gr}(X)=B_{i} \cap \operatorname{Gr}\left(Z\left(f_{i}\right)\right) ;
$$

2) si $i \in I$, il existe une injection $\sigma$ telle que $\operatorname{ord}_{\pi} \delta_{f_{i}, \sigma}\left(\varphi_{x}\right) \leq \operatorname{ord}_{\pi} \delta_{f_{i}, \sigma^{\prime}}\left(\varphi_{x}\right)$, pour toute injection $\sigma^{\prime} \neq \sigma$ et quel que soit $x \in B_{i}$.

Démonstration. - Si $x$ est un point de $\operatorname{Gr}(X)$ et $\kappa(x)$ son corps résiduel, on pose $\varphi_{x}:$ Spf $R_{\kappa(x)} \rightarrow X$ le $\mathbb{D}$-morphisme correspondant à $x$ par adjonction. Le lemme 4.5.2 ci-dessus implique que le cylindre $\operatorname{Gr}^{(e)}(X)$ peut être recouvert par un nombre fini de $c_{X} e$-cylindres $C, j \in J$, de $\operatorname{Gr}\left(\mathbb{B}_{R}^{N}\right)$ de la forme

$$
C:=\left\{x \in \operatorname{Gr}\left(\mathbb{B}_{R}^{N}\right) \mid(h \delta)\left(\varphi_{x}\right) \not \equiv 0 \bmod \left(\pi^{c_{X} e+1}\right)\right\} .
$$

La série formelle $h$ vérifie $h I \subset\left(f_{1}, \ldots, f_{N-d}\right)$ et $\left(f_{1}, \ldots, f_{N-d}\right) \in \Lambda_{N-d}$. La série formelle $\delta$ est un mineur de la matrice jacobienne des $f_{i}$. Fixons l'un des ensembles $C$. Soient $f$ le $(N-d)$-uplet $\left(f_{1}, \ldots, f_{N-d}\right)$. En reprenant les

TOME $132-2004-\mathrm{N}^{\mathrm{O}} 1$ 
notations de l'énoncé, on pose $\tau:\{1, \ldots, N-d\} \rightarrow\{1, \ldots, N-d\}$ l'injection telle que $\delta=\delta_{f, \tau}$. Soit $e^{\prime} \in \mathbf{N}$ tel que $e^{\prime} \leq c_{X} e$. Posons

$$
C_{\sigma, e^{\prime}}:=\left\{x \in C \mid \operatorname{ord}_{\pi} \delta_{f, \sigma}\left(\varphi_{x}\right)=e^{\prime} \text { et } \operatorname{ord}_{\pi} \delta_{f, \sigma^{\prime}}\left(\varphi_{x}\right) \geq e^{\prime}, \forall \sigma^{\prime}\right\}
$$

Remarquons que, comme $\delta_{f, \tau}\left(\varphi_{x}\right) \leq c_{X}$ e pour tout $x \in C$, ces ensembles $C_{\sigma, e^{\prime}}$ forment une partition de $C$. En outre, le lemme 4.3.7 implique que cette partition est finie. Il est clair que $C \cap \operatorname{Gr}(X)$ est un $c_{X}$ e-cylindre de $\operatorname{Gr}(X)$ et que $\operatorname{Gr}^{(e)}(X)$ est la réunion des $C \cap \mathrm{Gr}^{(e)}(X)$. Comme $h\left(\varphi_{x}\right) \neq 0$ pour tout $\varphi_{x} \in B$,

$$
\operatorname{Gr}(X) \cap C=\operatorname{Gr}\left(\operatorname{Spf} R\left\{x_{1}, \ldots, x_{N}\right\} /\left(f_{1}, \ldots, f_{N-d}\right)\right) \cap C .
$$

Les $C_{\sigma, e^{\prime}}$ forment le recouvrement souhaité.

LEMME 4.5.4. - Soit $X$ un $\mathbb{D}$-schéma formel admissible, purement de dimension relative $d$. Il existe $c_{X} \in \mathbf{N} \backslash\{0\}$ tel que, pour tout e et tout $n \in \mathbf{N}$ avec $n \geq c_{X} e$, on ait le résultat suivant :

1) l'application

$$
\theta_{n}: \pi_{n+1, X}\left(\operatorname{Gr}^{(e)}(X)\right) \longrightarrow \pi_{n, X}\left(\operatorname{Gr}^{(e)}(X)\right)
$$

est une fibration triviale par morceaux sur $\pi_{n, X}\left(\mathrm{Gr}^{(e)}(X)\right)$ de fibre $\mathbf{A}_{k}^{d}$.

2) En particulier, $\left[\pi_{n, X}\left(\operatorname{Gr}_{e}(X)\right)\right]=\left[\pi_{c_{X} e, X}\left(\operatorname{Gr}_{e}(X)\right)\right] \mathbf{L}^{d\left(n-c_{X} e\right)}$.

Démonstration. - 1) Le deuxième point est une conséquence directe de l'assertion 1) et du lemme 4.2.2.

2) Grâce au lemme 4.2.3, il nous suffit de montrer que pour tout $x \in \mathrm{Gr}^{(e)}(X)$ la fibre au-dessus de $\pi_{n, X}(x)$ est isomorphe à l'espace affine de dimension $d$ sur $\kappa(x)$, le corps résiduel de $x$.

Réduction géométrique. - La question étant locale, on peut supposer que $X$ est affine de la forme

$$
X=\operatorname{Spf} R\left\{x_{1}, \ldots, x_{N}\right\} / I \longleftrightarrow \mathbb{B}_{R}^{N} .
$$

Le lemme 4.5.3 permet de supposer que $I$ est engendré par

$$
f_{1}, \ldots, f_{N-d} \in R\left\{X_{1}, \ldots, X_{N}\right\},
$$

i.e. $X \hookrightarrow \mathbb{B}_{R}^{N}$ est d'intersection complète $(c f .4 .5 .3,2)$. En outre, grâce à l'assertion $4.5 .3,3$ ), on peut supposer qu'il existe un mineur $\delta$ d'ordre $N-d$ de la matrice jacobienne

$$
\Delta:=\left(\frac{\partial f_{i}}{\partial x_{j}}\right)_{1 \leq i \leq N-d, 1 \leq j \leq N-d}
$$

tel que, pour tout $x \in \mathrm{Gr}^{(e)}(X), \delta\left(\varphi_{x}\right)$ appartient à l'idéal engendré par $\pi^{e^{\prime}}$, avec $e^{\prime} \leq c_{X} e$ et que pour tout autre mineur $\delta^{\prime}$ d'ordre $N-d$ de $\Delta, \operatorname{ord}_{\pi} \delta^{\prime}\left(\varphi_{x}\right) \geq$ $\operatorname{ord}_{\pi} \delta\left(\varphi_{x}\right)$. Enfin, quitte à réordonner les $X_{j}$, on peut supposer que $\delta$ se calcule à partir des $N-d$ premières colonnes.

BULletin DE LA SOCiÉtÉ MATHÉmATiQUE DE FRANCE 
Étude des fibres de $\theta_{n}$. - Soit $x \in \mathrm{Gr}^{(e)}(X)$ de corps résiduel $k^{\prime}:=\kappa(x)$. Soit $\varphi$ : Spf $R_{k^{\prime}} \rightarrow X$ le $\mathbb{D}$-morphisme correspondant au point $x$. Si $y \in\left(R_{k^{\prime}}\right)^{N}$,

$$
f_{j}\left(\varphi+\pi^{n+1} y\right)=\pi^{n+1} D_{\varphi} f_{j} \cdot y+\pi^{2 n+2} F_{j}(y)
$$

pour tout $1 \leq j \leq N-d$, où $F_{j} \in R\left\{x_{1}, \ldots, x_{N}\right\}$. Soit $M$ la matrice adjointe de la sous-matrice de $\Delta$ définie par les $N-d$ premières colonnes. En particulier, cette matrice à coefficients dans $R\left\{x_{1}, \ldots, x_{N}\right\}$ vérifie

$$
M \cdot \Delta=\left(\delta I_{N-d}, H\right)
$$

où $H$ est une matrice $(N-d) \times d$ satisfaisant, grâce au choix de $e^{\prime}$, à

$$
H(\varphi) \equiv 0 \bmod \left(\pi^{e^{\prime}}\right) .
$$

En effet, soit $\widetilde{\Delta(\varphi)}$ la sous-matrice $N-d \times N-d$ de $\Delta(\varphi)$ formée des $N-d$ premières colonnes, que l'on note $\Delta_{i}(\varphi)$ pour $1 \leq i \leq N-d$. Soit $j$ un entier naturel tel que $1 \leq j \leq d$. Soit $H_{j}(\varphi)$ la $j$-ième colonne de $H(\varphi)$ (i.e. la $(N-d+j)$-ième colonne de $M(\varphi) \cdot \Delta(\varphi))$. Ce vecteur est solution du système

$$
\widetilde{\Delta(\varphi)} \cdot X=\delta \cdot \Delta_{j}(\varphi)
$$

où $X=\left(X_{i}\right)_{1 \leq i \leq N-d} \in\left(R_{k^{\prime}}\right)^{N-d}$ est un vecteur colonne. Ce système admet, pour tout $1 \leq j \leq d$, une solution, qui se décrit explicitement grâce à la règle de Cramer par

$$
X_{i}=\operatorname{det}\left(\Delta_{1}(\varphi), \ldots, \Delta_{i-1}(\varphi), \Delta_{j}(\varphi), \Delta_{i+1}(\varphi), \ldots, \Delta_{N-d}(\varphi)\right) .
$$

La fibre est alors décrite par le système

$$
\pi^{-e^{\prime}} M \cdot \Delta(\varphi) \cdot y+\pi^{n+1-e^{\prime}}(\ldots)=0 .
$$

Le lemme de Hensel ( $c f .[9]$, chap. III, $\S 4, \mathrm{n}^{\circ} 6$, cor. 2) assure alors que la fibre de $\theta_{n}$ au-dessus de $x$ est simplement l'ensemble des $y_{0} \in\left(k^{\prime}\right)^{N}$ vérifiant les équations linéaires

$$
\pi^{-e^{\prime}} M \cdot \Delta \cdot y_{0}=0 \in\left(k^{\prime}\right)^{N} .
$$

La fibre $\theta_{n}^{-1}(x)$ est donc naturellement munie d'une structure de $\kappa(x)$-espace vectoriel, de dimension $d$, exprimant les $N-d$ premières coordonnées en fonction de combinaisons linéaires des $d$-dernières, dont les coefficients sont paramétrés par les premières coordonnées de $\varphi$.

Remarque 4.5.5. - Soit $X$ un $\mathbb{D}$-schéma formel admissible, purement de dimension relative $d$. Alors la proposition 3.3.5, le lemme 4.4.2 et son corollaire vont entraîner que, ou bien le volume du lieu singulier de $X$ est nul, ou bien le volume de $\operatorname{Gr}(X)$ est nul. Ce dernier cas étant sans grand intérêt, nous ne le traitons pas explicitement dans les définitions et les preuves, bien que celles-ci soient encore valides dans ce cas.

TOME $132-2004-\mathrm{N}^{\mathrm{O}} 1$ 
Proposition 4.5.6. - Soit $X$ un $\mathbb{D}$-schéma formel admissible, purement de dimension relative $d$.

1) Pour tout cylindre $A \in \mathbf{C}_{X}$, la limite suivante existe dans $\widehat{M}_{k}$ :

$$
\widetilde{\mu}_{X}(A):=\lim _{e \rightarrow \infty} \mu_{0, X}\left(A \cap \operatorname{Gr}^{(e)}(X)\right) .
$$

2) Si $A \in \mathbf{C}_{0, X}$, alors $\widetilde{\mu}_{X}(A)$ et $\mu_{0, X}(A)$ coïncident dans $\bar{M}_{k}$.

3) L'application $A \mapsto \widetilde{\mu}_{X}(A)$ est $\sigma$-additive sur $\mathbf{C}_{X}$.

4) Pour $A$ et $B$ dans $\mathbf{C}_{X}$, on a

$$
\left\|\widetilde{\mu}_{X}(A \cup B)\right\| \leq \max \left(\left\|\widetilde{\mu}_{X}(A)\right\|,\left\|\widetilde{\mu}_{X}(B)\right\|\right) .
$$

Si $A \subset B$, on a $\left\|\widetilde{\mu}_{X}(A)\right\| \leq\left\|\widetilde{\mu}_{X}(B)\right\|$.

Démonstration. - Soit $A$ un cylindre de $\operatorname{Gr}(X)$ de rang $m$. Posons

$A^{(e)}:=A \cap \mathrm{Gr}^{(e)}(X), A_{(e)}:=\operatorname{Gr}(X) \backslash\left(A \cap \mathrm{Gr}^{(e)}(X)\right)=A \cap \pi_{e, X}^{-1}\left(\operatorname{Gr}_{e}\left(X_{\text {sing }}\right)\right)$.

Le lemme clé 4.5.4 assure que pour tout $e \in \mathbf{N}$ l'ensemble $A \cap \mathrm{Gr}^{(e)}(X)$ est un cylindre stable de $\operatorname{rang} \max \left(m, c_{X} e\right)$. Il nous faut donc montrer que la suite $\left(\mu_{0, X}\left(A \cap \operatorname{Gr}^{(e)}(X)\right)\right)_{e \in \mathbf{N}}$ converge dans $\widehat{M}_{k}$, i.e. est de Cauchy dans $\widehat{M}_{k}$. Soit $q \in \mathbf{Z}$. On veut montrer qu'il existe $e_{0} \in \mathbf{N}$ tel que pour tous $e^{\prime} \geq e \geq e_{0}$,

$$
\left[\pi_{n, X}\left(A^{(e)}\right)\right] \mathbf{L}^{-(n+1) d}-\left[\pi_{n, X}\left(A^{\left(e^{\prime}\right)}\right)\right] \mathbf{L}^{-(n+1) d} \in F^{q+1} \widehat{M}_{k} .
$$

Par additivité de [ ] sur les constructibles, pour $n \geq \max \left(m, c_{X} e, c_{X} e^{\prime}\right)$, on obtient

$$
\left[\pi_{n, X}\left(A^{(e)}\right)\right] \mathbf{L}^{-(n+1) d}=\left[\pi_{n, X}(A)\right] \mathbf{L}^{-(n+1) d}-\left[\pi_{n, X}\left(A_{(e)}\right)\right] \mathbf{L}^{-(n+1) d} .
$$

En particulier, ceci entraîne que

$$
\begin{aligned}
{\left[\pi_{n, X}\left(A^{(e)}\right)\right]-\left[\pi_{n, X}\left(A^{\left(e^{\prime}\right)}\right)\right] } & \\
=\left(\left[\pi_{n, X}\left(A^{(e)}\right) \cap \pi_{n, X}\left(A_{(e)}\right)\right]-\right. & {\left.\left[\pi_{n, X}\left(A^{\left(e^{\prime}\right)}\right) \cap \pi_{n, X}\left(A_{\left(e^{\prime}\right)}\right)\right]\right) } \\
& +\left(\left[\pi_{n, X}\left(A_{\left(e^{\prime}\right)}\right)\right]-\left[\pi_{n, X}\left(A_{(e)}\right)\right]\right)
\end{aligned}
$$

Si $e^{\prime} \geq e \geq \gamma(q)$, où $\gamma$ désigne la fonction de Greenberg pour $X_{\text {sing }}$, le lemme 4.4 .2 assure que

$$
\left[\pi_{n, X}\left(A_{\left(e^{\prime}\right)}\right)\right] \mathbf{L}^{-(n+1) d} \in F^{q+1} \widehat{M}_{k} \quad \text { et } \quad\left[\pi_{n, X}\left(A_{(e)}\right)\right] \mathbf{L}^{-(n+1) d} \in F^{q+1} \widehat{M}_{k}
$$

Ceci implique que la suite $\left(\left[\pi_{n, X}\left(A^{(e)}\right)\right] \mathbf{L}^{-(n+1) d}\right)_{e \in \mathbf{N}}$ est de Cauchy dans $\widehat{M}_{k}$. Les autres assertions sont vérifiées par $\mu_{0, X}$ et sont stables par passage à la limite. Elles découlent donc de la continuité de la norme.

La proposition suivante est un analogue du théorème 2 de [21].

BULLETIN DE LA SOCIÉTÉ MATHÉMATIQUE DE FRANCE 
Proposition 4.5.7. - Soit $A$ un cylindre de $\operatorname{Gr}(X)$ de rang $m$. La suite

$$
\left(\left[\pi_{n, X}(A)\right] \mathbf{L}^{-(n+1) d}\right)_{n \in \mathbf{N}}
$$

converge dans $\widehat{M}_{k}$ vers $\widetilde{\mu}_{X}(A)$.

Démonstration. - Soit $e \geq 0$, posons $A^{(e)}:=A \backslash \pi_{e, X}^{-1}\left(\operatorname{Gr}_{e}\left(X_{\text {sing }}\right)\right)$. Soit $\gamma$ la fonction de Greenberg pour $X_{\text {sing }}$. Le lemme 4.4 .2 assure que, pour tout $n \geq \max \left(\gamma(e), m, c_{X} e\right)$,

$$
\left[\pi_{n, X}(A)\right] \mathbf{L}^{-(n+1) d}-\left[\pi_{n, X}\left(A^{(\gamma(e))}\right)\right] \mathbf{L}^{-(n+1) d}
$$

appartient à $F^{e+1} M_{k}$. Par définition, $A^{(e)} \subset \mathrm{Gr}^{(e)}(X)$. Il découle du lemme 4.5.4 que $A^{(\gamma(e))}$ est stable de $\operatorname{rang} \max \left(m, c_{X} \gamma(e)\right)$. Par conséquent, pour tous $n$ et $n^{\prime} \geq \max \left(m, c_{X} \gamma(e)\right)$,

$$
\left[\pi_{n, X}\left(A^{(\gamma(e))}\right)\right] \mathbf{L}^{-(n+1) d}=\left[\pi_{n^{\prime}, X}\left(A^{(\gamma(e))}\right)\right] \mathbf{L}^{-\left(n^{\prime}+1\right) d} .
$$

On en déduit que pour tous $n$ et $n^{\prime} \geq \max \left(\gamma(e), m, c_{X} e\right)$,

$$
\left[\pi_{n, X}(A)\right] \mathbf{L}^{-(n+1) d}-\left[\pi_{n^{\prime}, X}(A)\right] \mathbf{L}^{-\left(n^{\prime}+1\right) d} \in F^{e+1} M_{k} .
$$

Ceci signifie que la suite

$$
\left(\left[\pi_{n, X}(A)\right] \mathbf{L}^{-(n+1) d}\right)_{n \in \mathbf{N}}
$$

est une suite de Cauchy et donc converge vers $\ell$ dans $\widehat{M}_{k}$. Comme, par définition, $\widetilde{\mu}_{X}\left(A^{(\gamma(e))}\right)=\left[\pi_{n, X}\left(A^{(\gamma(e))}\right)\right] \mathbf{L}^{-(n+1) d}$ pour $n \geq \max \left(m, c_{X} \gamma(e)\right)$, on en conclut que la suite de terme général $\widetilde{\mu}_{X}\left(A^{(\gamma(e))}\right)$ converge vers $\ell$ dans $\widehat{M}_{k}$. Comme, par définition, $\left(\widetilde{\mu}_{X}\left(A^{(\gamma(e))}\right)\right)$ converge vers $\widetilde{\mu}_{X}(A)$, on en déduit que $\mu_{X}(A)=\ell$.

\section{Morphismes tempérés}

Dans ce paragraphe, nous allons construire le principal exemple de ce que nous appellerons, plus tard, fonctions mesurables.

\subsection{Le jacobien d'un morphisme}

5.1.1. - Soit $Z \hookrightarrow X$ un sous- $\mathbb{D}$-schéma formel fermé de $X$, défini par un $O_{X^{-}}$ faisceau d'idéaux $I_{Z}$. Soit $x \in \operatorname{Gr}(X)$ de corps résiduel $\kappa(x) \supset k$. Si $k^{\prime} \supset \kappa(x)$ est une extension de corps parfaite, l'anneau $R_{k^{\prime}}$ est un anneau de valuation discrète complet, même si $R$ est d'inégales caractéristiques. Soit $\varphi: \operatorname{Spf} R_{k^{\prime}} \rightarrow X$ le $\mathbb{D}$-morphisme qui correspond à $x$. On dispose du morphisme canonique de $O_{X}$-modules

$$
\varphi^{*} I_{Z} \longrightarrow O_{R_{k^{\prime}}} \text {. }
$$

L'image de ce morphisme est un $O_{R_{k^{\prime}}}$-faisceaux d'idéaux de $O_{R_{k^{\prime}}}$. Deux cas se présentent alors : soit cet idéal est nul, soit il existe un entier naturel $n \in \mathbf{N}$ tel que $\varphi^{*} I_{Z} \cdot O_{R_{k^{\prime}}}=\pi^{n} \cdot O_{R_{k^{\prime}}}$. On appelle multiplicité de $x$ le long de $Z$ l'entier

TOME $132-2004-\mathrm{N}^{\mathrm{O}} 1$ 
défini par la valuation du générateur de l'idéal $\varphi^{*} I_{Z} \cdot O_{R_{k^{\prime}}}$ de $R_{k^{\prime}}$ si cet idéal est non nul, l'infini sinon. Il est clair que cette définition ne dépend pas du choix de $k^{\prime}$.

On définit alors l'application mult $Z: \operatorname{Gr}(X) \rightarrow \mathbf{N} \cup\{\infty\}$ comme l'application qui à $x \in \operatorname{Gr}(X)$ associe la multiplicité de $x$ le long de $Z$.

Lemme 5.1.2. - Soit $Z \hookrightarrow X$ un sous- $\mathbb{D}$-schéma formel fermé de $X$. On a les deux assertions suivantes:

1) Soit $U \hookrightarrow X$ un sous- $\mathbb{D}$-schéma formel ouvert de $X$ quasi-compact. Alors pour tout $x \in \operatorname{Gr}(U)$,

$$
\operatorname{mult}_{Z}(x)=\operatorname{mult}_{Z \cap U}(x) .
$$

2) Pour tout $n \in \mathbf{N}$, la fibre mult $_{Z}^{-1}(n)$ est un ensemble cylindrique de $\operatorname{Gr}(X)$.

3) Pour la fibre de l'infini, on a l'égalité

$$
\operatorname{mult}_{Z}^{-1}(\infty)=\operatorname{Gr}(Z) .
$$

4) Pour tout $n \in \mathbf{N} \backslash\{0\} \cup\{\infty\}, \pi_{0, X}\left(\right.$ mult $\left._{Z}^{-1}(n)\right) \subset Z_{0}$. Si le $\mathbb{D}$-schéma formel $Z \rightarrow \mathbb{D}$ est lisse, l'inclusion précédente est une égalité.

5) Si $Z^{\prime} \hookrightarrow X$ est un sous- $\mathbb{D}$-schéma formel fermé de $X$ contenu dans $Z$, alors, pour tout $x \in \operatorname{Gr}(X)$,

$$
\operatorname{mult}_{Z^{\prime}}(x) \leq \operatorname{mult}_{Z}(x) .
$$

6) Si $Z \subset X_{0}$, alors

$$
\operatorname{mult}_{Z}^{-1}(0)=\operatorname{Gr}(X \backslash Z), \quad \operatorname{mult}_{Z}^{-1}(1)=\operatorname{Gr}(X) \backslash \operatorname{Gr}(X \backslash Z), \quad \operatorname{Gr}(Z)=\varnothing .
$$

Démonstration. - Considérons le diagramme de $\mathbb{D}$-morphismes de schémas formels

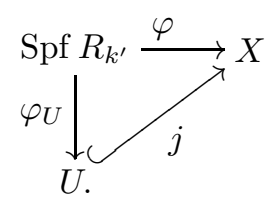

La commutativité de ce diagramme implique que les faisceaux d'idéaux de $O_{R_{k^{\prime}}} \varphi_{U}^{*} I_{Z \cap U} \cdot O_{R_{k^{\prime}}}$ et $\varphi^{*} I_{Z} \cdot O_{R_{k^{\prime}}}$ sont égaux. La première assertion en découle.

Pour les deux assertions suivantes, on peut supposer que $X$ est un schéma formel affine. Si $X:=\operatorname{Spf} R\left\{x_{1}, \ldots, x_{N}\right\} / I$, notons $A:=R\left\{x_{1}, \ldots, x_{N}\right\} / I$. Le sous-schéma formel $Z$ est alors défini par la donnée d'un idéal $J$ de $A$. Dans ce contexte, l'idéal $I_{Z} \cdot O_{R_{k^{\prime}}}$ est simplement l'idéal de $R_{k^{\prime}}$ engendré par les $g\left(\left(\varphi_{i}\right)_{1 \leq i \leq N}\right)$ pour tout $g \in J$ et où le $N$-uplet de $R_{k^{\prime}}^{N},\left(\varphi_{i}\right)_{1 \leq i \leq N}$, correspond au morphisme $\varphi$.

Soit $n \in \mathbf{N}$. Posons

$$
C_{>n}:=\pi_{Y, n}^{-1}\left(\operatorname{Gr}_{n}(Z)\right) .
$$

BULLETIN DE LA SOCIÉTÉ MATHÉMATIQUE DE FRANCE 
Il découle directement des définitions que

$$
C_{>n}=\left\{x \in \operatorname{Gr}(X) \mid \operatorname{mult}_{Z}(x) \geq n+1\right\} .
$$

Comme $C_{>n}$ est un cylindre de $\operatorname{Gr}(X)$, la deuxième assertion est une conséquence du fait que

$$
\left\{x \in \operatorname{Gr}(X) \mid \operatorname{mult}_{Z}(x)=n+1\right\}=C_{>n} \backslash C_{>n+1} .
$$

De la même manière, le dernier point se déduit facilement de la description ci-dessus.

LEMme 5.1.3. - Soit $h: Y \rightarrow X$ un $\mathbb{D}$-morphisme de schémas formels admissibles, réduits, tous deux de pure dimension relative $d$. Soit $Z \hookrightarrow X$ un sous- $\mathbb{D}-$ schéma formel. L'ensemble $h^{-1}(Z)$ peut être muni d'une structure de sous-Dschéma formel de $Y$. Soit $n \in \mathbf{N}$. Alors

$$
h\left(\operatorname{mult}_{h^{-1}(Z)}^{-1}(n)\right) \subset \operatorname{mult}_{Z}^{-1}(n) .
$$

En outre, si h est bijectif, cette inclusion est une égalité.

Démonstration. - Soit $x \in \operatorname{mult}_{h^{-1}(Z)}^{-1}(n)$. Soit $U \hookrightarrow X$ un ouvert affine de $X$ contenant $h(x)$. Soit $V \hookrightarrow Y$ un ouvert affine de $Y$ contenant $x$ et contenu dans $h^{-1}(U)$. L'assertion 1 du lemme 5.1.2 permet de supposer que $X$ et $Y$ sont des $\mathbb{D}$-schémas formels $t$ tf affines. On note $X:=\operatorname{Spf} A, Y:=\operatorname{Spf} B$ et $I$ désigne désormais un idéal de $A$. Pour alléger les notations, et quitte à étendre les scalaires, on peut supposer que $x$ est un point rationnel de $\operatorname{Gr}(Y)$. Soit $\varphi: \mathbb{D} \rightarrow Y$ le $\mathbb{D}$-morphisme de schémas formels lui correspondant par adjonction. Le schéma formel $h^{-1}(Z)$ est défini dans $Y$ par l'idéal de $B$, image de $h^{*} I$ dans $B$ par le morphisme canonique

$$
h^{*} I \longrightarrow h^{*} O_{X}=B
$$

induit par le morphisme d'inclusion $I \subset A$. La valeur de la fonction mult $_{h^{-1}(Z)}$ en $x$ est alors déterminée par l'image de $\varphi^{*} h^{*} I$ dans $\varphi^{*} O_{Y}=B=\varphi^{*} h^{*} O_{X}$. Or cette image détermine également la valeur de la fonction $\operatorname{mult}_{Z}^{-1}$ en $h(x)$.

5.1.4. Soit $y$ un point de $\operatorname{Gr}(Y) \backslash \operatorname{Gr}\left(Y_{\text {sing }}\right)$ et $\varphi: \operatorname{Spf} R_{k^{\prime}} \rightarrow Y$ le $\mathbb{D}$-morphisme correspondant, avec $k^{\prime}$ une clôture parfaite de $\kappa(y)$. On dispose de la suite exacte canonique

$$
h^{*} \Omega_{X / R}^{1} \longrightarrow \Omega_{Y / R}^{1} \longrightarrow \Omega_{Y / X}^{1} \rightarrow 0
$$

qui induit, en passant aux puissances extérieures $d$-ièmes, un $O_{Y}$-morphisme de modules

$$
h^{*} \Omega_{X / R}^{d} \longrightarrow \Omega_{Y / R}^{d},
$$

et, par image inverse via $\varphi$, un $R_{k^{\prime}}$-morphismes de modules (libres)

$$
\left(\varphi^{*}\left(h^{*} \Omega_{X / R}^{d}\right)\right) /(\text { torsion }) \longrightarrow\left(\varphi^{*} \Omega_{Y / R}^{d}\right) /(\text { torsion }) .
$$

TOME $132-2004-\mathrm{N}^{\mathrm{O}} 1$ 


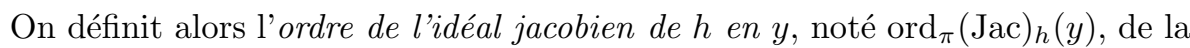
manière suivante. Le choix de $\varphi$ entraîne que

$$
L:=\left(h^{*} \Omega_{X / R}^{d}\right) /(\text { torsion })
$$

est un $R_{k^{\prime}}$-module libre de rang 1 . On déduit que l'image de

$$
M:=\left(\varphi^{*}\left(h^{*} \Omega_{X / R}^{d}\right)\right) /(\text { torsion })
$$

dans $L$ est égal soit à 0 , soit à $\pi^{n} L$, pour un certain $n \in \mathbf{N}$. On pose alors $\operatorname{ord}_{\pi}(\mathrm{Jac})_{h}(y)=\infty$ et $\operatorname{ord}_{\pi}(\mathrm{Jac})_{h}(y)=n$ respectivement.

5.1.5. Supposons ici que le $\mathbb{D}$-schéma formel $Y \rightarrow \mathbb{D}$ soit lisse. Dans ce cas, l'application $\operatorname{ord}_{\pi}(\mathrm{Jac})_{h}$ est définie par le $d$-ième idéal de Fitting de $\Omega_{Y / X}^{1}$, i.e. $\operatorname{Fitt}_{d}\left(\Omega_{Y / X}^{1}\right)$.

Soient $y \in \operatorname{Gr}(Y)$ et $\varphi \in Y\left(R_{k^{\prime}}\right)$ le $\mathbb{D}$-morphisme qui lui correspond. L'image du morphisme naturel

$$
M:=\left(\varphi^{*}\left(h^{*} \Omega_{X / R}^{1}\right)\right) /(\text { torsion }) \longrightarrow L:=\left(\varphi^{*} \Omega_{X / R}^{1}\right)
$$

est un $O_{R_{k^{\prime}}}$-Module, $M^{\prime}$, libre de rang au plus $d$. La suite exacte canonique

$$
0 \rightarrow M^{\prime} \longrightarrow L \longrightarrow \varphi^{*} \Omega_{Y / X}^{1} \rightarrow 0
$$

est une présentation de $\varphi^{*} \Omega_{Y / X}^{1}$, puisque, $Y$ étant lisse sur $\mathbb{D}, \Omega_{Y / \mathbb{D}}^{1}$ est localement libre. De cette suite, on déduit le morphisme

$$
\wedge^{d}\left(M^{\prime}\right) \otimes_{O_{R_{k^{\prime}}}} \wedge^{d} L^{\vee} \longrightarrow O_{R_{k^{\prime}}}
$$

dont l'image est, par définition, le $d$-ième idéal de Fitting de $\varphi^{*} \Omega_{Y / X}^{1}$. L'assertion découle donc de la relation

$$
\operatorname{Fitt}_{d}\left(\varphi^{*} \Omega_{Y / X}^{1}\right)=\varphi^{*} \operatorname{Fitt}_{d}\left(\Omega_{Y / X}^{1}\right) .
$$

Proposition 5.1.6. - Si $Y$ est lisse et si $Z_{h} \hookrightarrow X$ est le sous-D-schéma formel de $X$ défini par le d-ème idéal de Fitting de $\Omega_{Y / X}^{1}$, les applications $\operatorname{ord}_{\pi}(\mathrm{Jac})_{h}$ et mult $_{Z_{h}}$ sont égales sur $\operatorname{Gr}(Y)$.

Démonstration. — Ceci découle directement des définitions des deux fonctions.

Proposition 5.1 .7 (formule de composition). — Soient $X, Y, Z$ des $\mathbb{D}$-schémas formels admissibles, purement de dimension relative $d$. Soient $h: Y \rightarrow X$ et $g: Z \rightarrow Y$ des $\mathbb{D}$-morphismes de schémas formels. Soit $z \in \operatorname{Gr}(Z)$ tel que

$$
z \notin \operatorname{Gr}\left(Z_{\text {sing }}\right) \cup g^{-1}\left(\operatorname{Gr}\left(Y_{\text {sing }}\right)\right) \cup(h \circ g)^{-1}\left(\operatorname{Gr}\left(X_{\text {sing }}\right)\right) .
$$

On a alors l'égalité suivante :

$$
\operatorname{ord}_{\pi}(\mathrm{Jac})_{h \circ g}(z)=\operatorname{ord}_{\pi}(\mathrm{Jac})_{h} \circ g(z)+\operatorname{ord}_{\pi}(\mathrm{Jac})_{g}(z) .
$$


Démonstration. - Soit $\varphi \in \operatorname{Gr}(Z)\left(k^{\prime}\right)$ un $\mathbb{D}$-morphisme correspondant à $z$. L'ordre de l'idéal jacobien de $h \circ g$ est donné par le morphisme

$$
(h \circ g)^{*} \Omega_{X / R}^{d} \longrightarrow \Omega_{Z / R}^{d} .
$$

La fonction $\operatorname{ord}_{\pi}(\mathrm{Jac})_{h}$ est décrite par le morphisme

$$
h^{*} \Omega_{X / R}^{d} \longrightarrow \Omega_{Y / R}^{d} .
$$

Enfin, l'ordre de l'idéal jacobien du morphisme $g$ est donné par

$$
g^{*} \Omega_{Y / R}^{d} \longrightarrow \Omega_{Z / R}^{d}
$$

Ces morphismes induisent deux $O_{R_{k^{\prime}}}$-morphismes $\varphi^{*}(h \circ g)^{*} \Omega_{X / R}^{d} \rightarrow \varphi^{*} \Omega_{Z / R}^{d}$ et

$$
(g \circ \varphi)^{*}\left(h^{*} \Omega_{X / R}^{d}\right) \longrightarrow(g \circ \varphi)^{*} \Omega_{Y / R}^{d} \stackrel{=}{\longrightarrow} \varphi^{*}\left(g^{*} \Omega_{Y / R}^{d}\right) \longrightarrow \varphi^{*} \Omega_{Z / R}^{d}
$$

qui sont égaux. Ceci entraîne en particulier que l'ordre de l'idéal jacobien de $h \circ g$ en $z$ est infini si et seulement si l'ordre de l'idéal jacobien de $h$ est infini en $g(z)$ ou si l'ordre du jacobien de $g$ est infini en $z$.

Supposons désormais que l'ordre de l'idéal jacobien de $h \circ g$ en $z$ est fini. Posons $L:=(g \circ \varphi)^{*} \Omega_{Y / R}^{d} /($ torsion $)$. L'image de $M:=(h \circ g \circ \varphi)^{*} \Omega_{X / R}^{d} /($ torsion $)$ dans $L$ est un $O_{R_{k^{\prime}}}$-Module $M^{\prime}$ libre de type fini. Il existe $n \in \mathbf{N}$ tel que $M^{\prime}=\pi^{n} L$. De même l'image $L^{\prime}$ de $L$ dans $K:=\varphi^{*} \Omega_{Z / R}^{d} /$ (torsion) est canoniquement isomorphe à $L^{\prime}=\pi^{m} K$. L'égalité des deux morphismes précédents prouve la formule

$$
\operatorname{ord}_{\pi}(\mathrm{Jac})_{h \circ g}(y)=m+n
$$

qui est l'égalité voulue.

5.2. La définition. - Soient $Y$ et $X$ deux $\mathbb{D}$-schémas formels admissibles, purement de dimension relative $d$. Soit $h: Y \rightarrow X$ un $\mathbb{D}$-morphisme de schémas formels. On appelle ordre de l'idéal jacobien de $h$ l'application

$$
\operatorname{ord}_{\pi}(\mathrm{Jac})_{h}: \operatorname{Gr}(Y) \backslash \operatorname{Gr}\left(Y_{\text {sing }}\right) \longrightarrow \mathbf{N} \cup\{\infty\}
$$

qui à $y \in \operatorname{Gr}(Y) \backslash \operatorname{Gr}\left(Y_{\text {sing }}\right)$ associe l'entier $\operatorname{ord}_{\pi}(\operatorname{Jac})_{h}(y)$ défini ci-dessus.

On appelle lieu sauvage de $h$ le sous-ensemble de $\operatorname{Gr}(Y)$ :

$$
\Sigma_{h}:=\operatorname{Gr}\left(Y_{\text {sing }}\right) \cup h^{-1}\left(\operatorname{Gr}\left(X_{\text {sing }}\right)\right) \cup\left\{y \in \operatorname{Gr}(Y) \mid \operatorname{ord}_{\pi}(\operatorname{Jac})_{h}(y)=\infty\right\} .
$$

On notera

$$
\Pi_{h}=\left\{y \in \operatorname{Gr}(Y) \mid \operatorname{ord}_{\pi}(\mathrm{Jac})_{h}(y)=\infty\right\} .
$$

Soit $B$ un sous-ensemble de $\operatorname{Gr}(Y)$. On dit que le morphisme $h$ est tempéré sur $B$ si $B \cap \Sigma_{h}$ est mesurable de mesure nulle. On dit que $h$ est tempéré si $h$ est tempéré sur $\operatorname{Gr}(Y)$, i.e. si le lieu sauvage de $h$ est de mesure nulle. On appelle lieu tempéré de $h$ le complémentaire de $\Sigma_{h}$ dans $\operatorname{Gr}(Y)$.

TOME $132-2004-\mathrm{N}^{\mathrm{O}} 1$ 
Supposons que le $\mathbb{D}$-schéma formel $Y \rightarrow \mathbb{D}$ soit lisse. Soit $B$ un sous-ensemble de $\operatorname{Gr}(Y)$ contenu dans le lieu tempéré de $h$. L'ensemble $B$ est alors contenu dans l'ind-cylindre

$$
\left(\bigcup_{e \in \mathbf{N}} h^{-1}\left(\operatorname{Gr}^{(e)}(X)\right) \cap\left(\bigcup_{e^{\prime} \in \mathbf{N}}\left\{y \in \operatorname{Gr}(Y) \mid \operatorname{ord}_{\pi}(\operatorname{Jac})_{h}(y)=e^{\prime}\right\}\right) .\right.
$$

Nous noterons

$$
\Delta_{e, e^{\prime}}=h^{-1}\left(\operatorname{Gr}^{(e)}(X)\right) \cap\left\{y \in \operatorname{Gr}(Y) \mid \operatorname{ord}_{\pi}(\mathrm{Jac})_{h}(y)=e^{\prime}\right\} .
$$

En particulier, si $B$ est un cylindre, il est contenu dans la réunion d'un nombre fini de tels $\Delta_{e, e^{\prime}}$.

ExEMPLES 5.2.1. - Les isomorphismes, les immersions ouvertes, les éclatements admissibles sont des exemples de morphismes tempérés.

\section{Ensembles mesurables}

Supposons désormais que $X$ est un $\mathbb{D}$-schéma formel admissible, purement de dimension relative $d$.

6.1. La définition. - Soit $A$ un sous-ensemble de $\operatorname{Gr}(X)$.

DÉfinition 6.1.1. - On dit que $A$ est mesurable dans $\operatorname{Gr}(X)$ si, pour tout $\varepsilon>0$, il existe un ensemble $I$ au plus dénombrable et une famille de cylindres $\left(A_{i}\right)_{i \in I \cup\{0\}}$ tels que :

1) $A \Delta A_{0} \subset \bigcup_{i \in I} A_{i}$, où $\Delta$ désigne la différence symétrique.

2) $\left\|\mu\left(A_{i}\right)\right\|<\varepsilon$, pour tout $i \in I$.

Dans ce cas, on dit qu'une telle famille de cylindres $\left(A_{i}\right)_{i \in I \cup\{0\}}$, que l'on note $\left(A_{0} ;\left(A_{i}\right)_{i \in I}\right)$, est une $\varepsilon$-approximation cylindrique de $A$. Le cylindre $A_{0}$ est appelé la partie principale de l'approximation cylindrique. On dit que $A$ est fortement mesurable si l'on peut choisir $A_{0} \subset A$, pour tout $\varepsilon>0$.

Exemple 6.1.2. - Tout cylindre $C$ de $\operatorname{Gr}(X)$ est mesurable (même fortement mesurable). En effet, si $\varepsilon>0$, la famille de cylindres $\left(C ;(\varnothing)_{i \in \mathbf{N}}\right)$ est une $\varepsilon$ approximation cylindrique de $C$.

On désignera par $\mathbf{D}_{X}\left(\right.$ resp. $\mathbf{D}_{X}^{f}$ ) l'ensemble des ensembles mesurables (resp. fortement mesurables) de $\operatorname{Gr}(X)$.

Proposition 6.1.3. - Les ensembles de parties $\mathbf{D}_{X}$ et $\mathbf{D}_{X}^{f}$ vérifient les propriétés suivantes :

1) $\mathbf{D}_{X}^{f} \subset \mathbf{D}_{X}$.

2) Les ensembles $\operatorname{Gr}(X)$ et $\varnothing$ appartiennent à $\mathbf{D}_{X}^{f}$.

3) L'ensemble $\mathbf{D}_{X}$ (resp. $\mathbf{D}_{X}^{f}$ ) est stable par intersection finie.

BULLETIN DE LA SOCIÉtÉ MATHÉMATIQUE DE FRANCE 
4) L'ensemble $\mathbf{D}_{X}$ (resp. $\mathbf{D}_{X}^{f}$ ) est stable par réunion finie.

5) L'ensemble $\mathbf{D}_{X}$ est stable par passage au complémentaire.

6) Si $A$ et $B$ sont deux parties mesurables de $G(X)$, alors $A \backslash B$ est encore une partie mesurable de $\operatorname{Gr}(X)$.

En particulier, $\mathbf{D}_{X}$ est un anneau booléen.

Démonstration. - Les ensembles $\operatorname{Gr}(X)$ et $\varnothing$ sont des cylindres, donc des ensembles fortement mesurables. Soit $\varepsilon>0$. Pour les assertions 3 ) et 4 ), un raisonnement par récurrence permet de se ramener facilement au cas de la réunion de deux ensembles mesurables $A$ et $B$. Soit $\varepsilon>0$. Soient $\left(A_{0} ;\left(A_{i}\right)_{i \in I}\right)$ et soit $\left(B_{0} ;\left(B_{j}\right)_{j \in J}\right)$ des $\varepsilon$-approximations cylindriques de $A$ et $B$ respectivement. Alors la famille $\left(A_{0} \cup B_{0} ;\left(A_{i}\right)_{i} \cup\left(B_{j}\right)_{j}\right)$ est une $\varepsilon$-approximation cylindrique de $A \cup B$. De même, la famille $\left(A_{0} \cap B_{0} ;\left(A_{i}\right)_{i} \cup\left(B_{j}\right)_{j}\right)$ est une $\varepsilon$-approximation cylindrique de $A \cap B$. Le point 5$)$ découle de l'égalité suivante

$$
A \Delta A_{0}=(\operatorname{Gr}(X) \backslash A) \Delta\left(\operatorname{Gr}(X) \backslash A_{0}\right) .
$$

Le point 6 ) est une conséquence formelle des points 3$)$ et 5 ). Toutefois, il est également clair que la suite de cylindres définie par $C_{0}:=A_{0} \backslash B_{0}$, les $A_{i}$ pour tout $i \in I$ et les $B_{j}$ pour tout $j \in J$ est une $\varepsilon$-approximation cylindrique de $C:=A \backslash B$.

\subsection{La mesure motivique pour les ensembles mesurables.}

Lemme 6.2.1. - Soit $A$ un ensemble mesurable de $\operatorname{Gr}(X)$. Soit $\varepsilon>0$ et $\varepsilon^{\prime}>0$ deux nombres réels. Si $\left(A_{0} ;\left(A_{i}\right)_{i \in I}\right)$ est une $\varepsilon$-approximation cylindrique de $A$ et $\left(A_{0}^{\prime} ;\left(A_{i}^{\prime}\right)_{i \in I}\right)$ une $\varepsilon^{\prime}$-approximation cylindrique de $A$, alors

$$
\left\|\widetilde{\mu}_{X}\left(A_{0}\right)-\widetilde{\mu}_{X}\left(A_{0}^{\prime}\right)\right\|<\max \left(\varepsilon, \varepsilon^{\prime}\right) .
$$

Démonstration. - On omettra l'indice $X$ dans la preuve. La preuve est l'adaptation d'une preuve de Batyrev [1], établie pour démontrer un résultat analogue. Soit $A$ un ensemble mesurable. Soient $\varepsilon>0$ et $\varepsilon^{\prime}>0$ deux nombres réels. Soient $\left(A_{0}(\varepsilon) ;\left(A_{i}(\varepsilon)\right)_{i \in I(\varepsilon)}\right)$ et $\left(A_{0}^{\prime}\left(\varepsilon^{\prime}\right) ;\left(A_{j}^{\prime}\left(\varepsilon^{\prime}\right)\right)_{j \in I^{\prime}\left(\varepsilon^{\prime}\right)}\right)$ deux approximations cylindriques de $A$. Par définition, on a la relation

$$
A_{0}(\varepsilon) \Delta A_{0}^{\prime}\left(\varepsilon^{\prime}\right) \subset\left(\bigcup_{i \in I(\varepsilon)} A_{i}(\varepsilon)\right) \cup\left(\bigcup_{i \in I^{\prime}\left(\varepsilon^{\prime}\right)} A_{i}^{\prime}\left(\varepsilon^{\prime}\right)\right) .
$$

Comme $A_{0}(\varepsilon) \Delta A_{0}^{\prime}\left(\varepsilon^{\prime}\right)$ est un cylindre, le lemme 4.3.7 assure qu'il existe deux entiers $L(\varepsilon)$ et $L\left(\varepsilon^{\prime}\right)$ tels que

$$
A_{0}(\varepsilon) \Delta A_{0}^{\prime}\left(\varepsilon^{\prime}\right) \subset\left(\bigcup_{i=1}^{L(\varepsilon)} A_{i}(\varepsilon)\right) \cup\left(\bigcup_{i=1}^{L\left(\varepsilon^{\prime}\right)} A_{i}^{\prime}\left(\varepsilon^{\prime}\right)\right) .
$$

Les propriétés de la norme sur $\widehat{M}_{k}$ donnent la relation

$$
\left\|\widetilde{\mu}\left(A_{0}(\varepsilon) \Delta A_{0}^{\prime}\left(\varepsilon^{\prime}\right)\right)\right\| \leq \max \left(\varepsilon, \varepsilon^{\prime}\right) .
$$

TOME $132-2004-\mathrm{N}^{\mathrm{O}} 1$ 
En utilisant les inclusions

$$
\begin{aligned}
& A_{0}(\varepsilon) \backslash\left(A_{0}(\varepsilon) \cap A_{0}^{\prime}\left(\varepsilon^{\prime}\right)\right) \subset A_{0}\left(\varepsilon^{\prime}\right) \Delta A_{0}^{\prime}\left(\varepsilon^{\prime}\right), \\
& A_{0}^{\prime}\left(\varepsilon^{\prime}\right) \backslash\left(A_{0}(\varepsilon) \cap A_{0}^{\prime}\left(\varepsilon^{\prime}\right)\right) \subset A_{0}(\varepsilon) \Delta A_{0}^{\prime}\left(\varepsilon^{\prime}\right),
\end{aligned}
$$

on obtient les inégalités

$$
\begin{aligned}
& \left\|\widetilde{\mu}\left(A_{0}(\varepsilon) \backslash\left(A_{0}(\varepsilon) \cap A_{0}^{\prime}\left(\varepsilon^{\prime}\right)\right)\right)\right\| \leq \max \left(\varepsilon, \varepsilon^{\prime}\right), \\
& \left\|\widetilde{\mu}\left(A_{0}^{\prime}(\varepsilon) \backslash\left(A_{0}(\varepsilon) \cap A_{0}^{\prime}\left(\varepsilon^{\prime}\right)\right)\right)\right\| \leq \max \left(\varepsilon, \varepsilon^{\prime}\right) .
\end{aligned}
$$

En utilisant la proposition 4.5.6 et les décompositions

$$
\begin{aligned}
& A_{0}(\varepsilon)=\left(A_{0}(\varepsilon) \backslash\left(A_{0}(\varepsilon) \cap A_{0}^{\prime}\left(\varepsilon^{\prime}\right)\right)\right) \sqcup\left(A_{0}(\varepsilon) \cap A_{0}^{\prime}\left(\varepsilon^{\prime}\right)\right), \\
& A_{0}^{\prime}(\varepsilon)=\left(A_{0}^{\prime}(\varepsilon) \backslash\left(A_{0}(\varepsilon) \cap A_{0}^{\prime}\left(\varepsilon^{\prime}\right)\right)\right) \sqcup\left(A_{0}(\varepsilon) \cap A_{0}^{\prime}\left(\varepsilon^{\prime}\right)\right),
\end{aligned}
$$

on obtient

$$
\left\|\widetilde{\mu}\left(A_{0}(\varepsilon)\right)-\widetilde{\mu}\left(A_{0}^{\prime}\left(\varepsilon^{\prime}\right)\right)\right\| \leq \max \left(\varepsilon, \varepsilon^{\prime}\right) .
$$

ThÉORÈme 6.2.2. — Soit $X$ un $\mathbb{D}$-schéma formel admissible, purement de dimension relative $d$. Il existe une unique mesure $\sigma$-additive $\mu_{X}: \mathbf{D}_{X} \rightarrow \widehat{M}_{k} q u i$ coïncide avec l'application $\widetilde{\mu}_{X}$ sur $\mathbf{C}_{X}$ et vérifie

$$
\left\|\mu_{X}(A \cup B)\right\| \leq \max \left(\left\|\mu_{X}(A)\right\|,\left\|\mu_{X}(B)\right\|\right)
$$

pour $A$ et $B$ dans $\mathbf{D}_{X}$. Si $A \subset B$, on a $\left\|\mu_{X}(A)\right\| \leq\left\|\mu_{X}(B)\right\|$.

Si $\left(A_{0} ;\left(A_{i}\right)_{i \in I}\right)$ est une $\varepsilon$-approximation cylindrique de $A$, elle est donnée par la formule

$$
\mu_{X}(A):=\lim _{\varepsilon \rightarrow 0} \widetilde{\mu}_{X}\left(A_{0}(\varepsilon)\right) .
$$

En particulier, la limite ne dépend pas du choix des approximations cylindriques de $A$.

Démonstration. - On omettra l'indice $X$ dans la preuve. L'existence de l'application $\mu$ découle directement du lemme 6.2.1 ci-dessus. En effet, si $\left(A_{0}(\varepsilon) ;\left(A_{i}(\varepsilon)\right)_{i \in I}\right)$ est une $\varepsilon$-approximation cylindrique de $A$, la famille $\left(\widetilde{\mu}\left(A_{0}(\varepsilon)\right)\right)_{\varepsilon>0}$ est de Cauchy, donc converge dans $\widehat{M}_{k}$. En outre, soient $a$ et $a^{\prime}$ les limites respectives des suites $\left(\widetilde{\mu}\left(A_{0}(1 / n)\right)_{n \in \mathbf{N}}\right.$ et $\left(\widetilde{\mu}\left(A_{0}^{\prime}(1 / m)\right)_{m \in \mathbf{N}}\right.$. On a l'inégalité

$$
\begin{array}{r}
\left\|a-a^{\prime}\right\| \leq \max \left(\left\|a-\widetilde{\mu}\left(A_{0}(1 / n)\right)\right\|,\left\|\widetilde{\mu}\left(A_{0}(1 / n)\right)-\widetilde{\mu}\left(A_{0}^{\prime}(1 / m)\right)\right\|,\right. \\
\left.\left\|a^{\prime}-\widetilde{\mu}\left(A_{0}(1 / m)\right)\right\|\right) .
\end{array}
$$

Ceci implique que les deux limites coïncident.

Soit $C$ un cylindre de $\operatorname{Gr}(X)$. Le fait que, pour tout $\varepsilon>0$, on peut choisir une $\varepsilon$-approximation de $C$ de partie principale égale à $C$ entraîne que $\mu$ et $\widetilde{\mu}$ coïncident.

Les inégalités vérifiées par $\mu$ font l'objet du lemme 6.3 .3 , la $\sigma$-additivité celui du lemme 6.3.4.

BULletin DE LA SOCiÉtÉ MATHÉmATiQUe DE FRANCE 


\subsection{Les propriétés générales}

Lemme 6.3.1. - Soient $A$ et $B$ deux ensembles mesurables de $\operatorname{Gr}(X)$.

1) Si $A \cap B=\varnothing$, alors, pour tout $\varepsilon>0$, il existe une $\varepsilon$-approximation cylindrique de $A$, de partie principale $A_{0}$, et une $\varepsilon$-approximation cylindrique de $B$, de partie principale $B_{0}$, telle que $A_{0} \cap B_{0}=\varnothing$.

2) Si $A \subset B$, alors, pour tout $\varepsilon>0$, il existe une $\varepsilon$-approximation cylindrique de $A$, de partie principale $A_{0}$, et une $\varepsilon$-approximation cylindrique de $B$, de partie principale $B_{0}$, telle que $A_{0} \subset B_{0}$. En particulier, si $B$ est fortement mesurable, on peut supposer que $A_{0} \subset B_{0} \subset B$.

Démonstration. - Soient $\varepsilon>0,\left(A_{0}^{\prime} ;\left(A_{i}^{\prime}\right)_{i \in I}\right)$ et $\left(B_{0}^{\prime} ;\left(B_{i}^{\prime}\right)_{i \in J}\right)$ des $\varepsilon$-approximations cylindriques de $A$ et $B$.

1) La suite de cylindres de $\operatorname{Gr}(X)$ définie par $A_{0}:=A_{0}^{\prime} \backslash B_{0}^{\prime}$, par les $A_{i}$ pour tout $i \in I$ et par les $B_{j}^{\prime}$ pour tout $j \in J$ (par $B_{j}^{\prime}$ pour tout $j \in J \cup\{0\}$ ) est une $\varepsilon$-approximation cylindrique de $A$ (de $B$ ). Ces deux suites vérifient $6.3 .1,1$ ).

2) La suite de cylindres définie par $A_{0}:=A_{0}^{\prime} \cap B_{0}^{\prime}$, par les $A_{j}^{\prime}$ pour tout $j \neq 0$ et les $B_{j}^{\prime}$ pour tout $j \neq 0$ (resp. par les $B_{j}^{\prime}$ ) est une $\varepsilon$-approximation cylindrique de $A$ (resp. $B$ ). Ces deux suites satisfont à la condition $6.3 .1,2$ ).

Proposition 6.3.2. - Soient $n \geq 1$ et $\left(A^{(i)}\right)_{1 \leq i \leq n}$ une suite d'ensembles mesurables de $\operatorname{Gr}(X)$.

1) Si les $A^{(i)}$ sont deux à deux disjoints, alors, pour tout $1 \leq i \leq n$, pour tout $\varepsilon>0$, il existe une $\varepsilon$-approximation cylindrique de $A^{(i)}$, telle que pour tout couple $(i, j)$, avec $i \neq j, A_{0}^{(i)}(\varepsilon) \cap A_{0}^{(j)}(\varepsilon)=\varnothing$.

2) Si $A^{(1)} \subset \cdots \subset A^{(n)}$, alors, pour tout $1 \leq i \leq n$, pour tout $\varepsilon>0$, il existe une $\varepsilon$-approximation cylindrique de $A^{(i)}$, telle que $A_{0}^{(1)}(\varepsilon) \subset \cdots \subset A_{0}^{(n)}(\varepsilon)$.

Démonstration. - Prouvons les résultats par récurrence sur $n$. Considérons $n+1$ parties mesurables de $\operatorname{Gr}(X)$ deux à deux disjointes. Par hypothèse de récurrence, on peut trouver, pour tout $1 \leq i \leq n$, des $\varepsilon$-approximations cylindriques de $A^{(i)}$ de parties principales deux à deux disjointes. La proposition $6.1 .3,4)$ assure qu'il existe une $\varepsilon$-approximation cylindrique de $\bigcup_{1 \leq i \leq n} A^{(i)}$ de partie principale $\bigcup_{1 \leq i \leq} A_{0}^{(i)}(\varepsilon)$. Le lemme $\left.6.3 .1,1\right)$ construit alors une $\varepsilon$ approximation cylindrique de $A^{(n+1)}$ de partie principale d'intersection vide avec $\bigcup_{1 \leq i \leq} A_{0}^{(i)}(\varepsilon)$.

De même, si $A^{(1)} \subset \cdots \subset A^{(n+1)}$, on peut trouver, par hypothèse de récurrence, pour tout $1 \leq i \leq n$, des $\varepsilon$-approximations cylindriques de $A^{(i)}$ telles que $A_{0}^{(1)}(\varepsilon) \subset \cdots \subset A_{0}^{(\bar{n})}(\varepsilon)$. L'assertion 6.3.1,2) assure que l'on peut construire une $\varepsilon$-approximation cylindrique de $A^{(n+1)}$ telle que $\bigcup_{1 \leq i \leq} A_{0}^{(i)}(\varepsilon) \subset A_{0}^{(n+1)}(\varepsilon)$. 
Lemme 6.3.3. - Soient $A$ et $B$ deux ensembles mesurables de $\operatorname{Gr}(X)$. Si $A$ est contenu dans $B$, alors $\left\|\mu_{X}(A)\right\| \leq\left\|\mu_{X}(B)\right\|$. En outre,

$$
\left\|\mu_{X}(A \cup B)\right\| \leq \max \left(\left\|\mu_{X}(A)\right\|,\left\|\mu_{X}(B)\right\|\right) .
$$

Démonstration. - Le lemme 6.3.1 et la proposition 6.1.3 assurent qu'il existe des approximations cylindriques $\left(A_{0} ;\left(A_{i}\right)_{i}\right)$ de $A$ et $\left(B_{0} ;\left(B_{j}\right)_{j}\right)$ de $B$ telles que $A_{0} \subset B_{0}$, si $A \subset B$, et telle que $A_{0} \cup B_{0}$ est la partie principale d'une approximation de $A \cup B$. L'assertion découle donc de la continuité de la norme.

Proposition 6.3.4. - Soit $\left(A^{(i)}\right)_{i \in \mathbf{N}}$ une suite d'ensembles mesurables de $\operatorname{Gr}(X)$.

1) Si la suite $\left(\mu_{X}\left(A^{(i)}\right)\right)_{i \in \mathbf{N}}$ tend vers 0 dans $\widehat{M}_{k}$, alors $A:=\bigcup_{i \in \mathbf{N}} A^{(i)}$ est mesurable dans $\operatorname{Gr}(X)$. Si l'on suppose en outre les $A^{(i)}$ fortement mesurables, alors $A$ est fortement mesurable.

2) Si les $A^{(i)}$ sont deux à deux disjoints et si l'on suppose que $A:=\bigcup_{i \in \mathbf{N}} A^{(i)}$ est un ensemble mesurable de $\operatorname{Gr}(X)$, alors $\mu_{X}(A)=\sum_{i \in \mathbf{N}} \mu_{X}\left(A^{(i)}\right)$.

Démonstration. - 1) On posera $\mu$ pour $\mu_{X}$ dans cette preuve. Soit $\varepsilon>0$. Il s'agit de construire une $\varepsilon$-approximation cylindrique de $\bigcup_{i \in \mathbf{N}} A^{(i)}$. Pour tout $\varepsilon^{\prime}>0$, pour tout $i \in \mathbf{N}$, soit $\left(A_{0}^{(i)}\left(\varepsilon^{\prime}\right) ;\left(A_{j}^{(i)}\left(\varepsilon^{\prime}\right)\right)_{j \in I_{i}\left(\varepsilon^{\prime}\right)}\right)$ une $\varepsilon^{\prime}$-approximation cylindrique de $A^{(i)}$. Le lemme 6.2.1 implique que, pour tout $\varepsilon^{\prime}>0$ et $n \in \mathbf{N}$,

$$
\left\|\mu\left(A_{0}^{(i)}\left(\varepsilon^{\prime}\right)\right)-\mu\left(A_{0}^{(i)}(1 / n)\right)\right\| \leq \max \left(\varepsilon^{\prime}, 1 / n\right) .
$$

Ceci entraîne que, pour tout $i \in \mathbf{N}$,

$$
\left\|\mu\left(A_{0}^{(i)}(\varepsilon / 4)\right)\right\| \leq \max \left(\frac{1}{2} \varepsilon,\left\|\mu\left(A^{(i)}\right)\right\|\right) .
$$

Enfin, par hypothèse, il existe $i_{0} \in \mathbf{N}$ tel que, pour tout $i>i_{0},\left\|\mu\left(A^{(i)}\right)\right\|<\frac{1}{2} \varepsilon$. Donc

$$
\left\|\mu\left(A_{0}^{(i)}\left(\frac{1}{4} \varepsilon\right)\right)\right\|<\varepsilon .
$$

La suite de cylindres définie par $B_{0}(\varepsilon):=\bigcup_{i=0}^{i_{0}} A_{0}^{(i)}\left(\frac{1}{4} \varepsilon\right)$, par les $A_{j}^{(i)}\left(\frac{1}{4} \varepsilon\right)$ pour $i \in \mathbf{N}$ et $j \in I_{i}$, et les $A_{0}^{\left(i+i_{0}+1\right)}\left(\frac{1}{4} \varepsilon\right)$ pour tout $i \in \mathbf{N}$, est une $\varepsilon$-approximation cylindrique de $B:=\bigcup A^{(i)}$, grâce au choix de $i_{0}$. Si les $A^{(i)}$ sont fortement mesurables, on peut supposer que $A_{0}^{(i)} \subset A^{(i)}$, de sorte que $B_{0} \subset B$.

2) Pour tout $\delta>0,\left(A_{0}(\delta) ;\left(A_{i}(\delta)\right)_{i \in I(\delta)}\right)$ désigne une $\delta$-approximation cylindrique de $A$ et $\left(A_{0}^{(j)}(\delta) ;\left(A_{i}^{(j)}(\delta)\right)_{i \in I_{j}(\delta)}\right)$ une $\delta$-approximation cylindrique de $A^{(j)}$ pour tout $j \in \mathbf{N}$. 
Soit $1>\varepsilon>0$. Par définition de la mesure $\mu_{X}$, il existe $\varepsilon>\varepsilon^{\prime}>0$ tel que $\left\|\mu_{X}(A)-\mu_{X}\left(A_{0}\left(\varepsilon^{\prime}\right)\right)\right\|<\varepsilon$. Comme $A \Delta A_{0}\left(\varepsilon^{\prime}\right) \subset \bigcup_{i \in I\left(\varepsilon^{\prime}\right)} A_{i}\left(\varepsilon^{\prime}\right)$,

$$
A_{0}\left(\varepsilon^{\prime}\right) \subset\left(\bigcup_{j \in \mathbf{N}} A^{(j)}\right) \cup\left(\bigcup_{i \in I\left(\varepsilon^{\prime}\right)} A_{i}\left(\varepsilon^{\prime}\right)\right) .
$$

De même, comme $A^{(j)} \Delta A_{0}^{(j)}\left(\varepsilon^{\prime}\right) \subset \bigcup_{i \in I_{j}\left(\varepsilon^{\prime}\right)} A_{i}^{(j)}\left(\varepsilon^{\prime}\right)$,

$$
A_{0}\left(\varepsilon^{\prime}\right) \subset\left(\bigcup_{\substack{j \in \mathbf{N} \\ i \in I_{j}\left(\varepsilon^{\prime}\right) \cup\{0\}}} A_{i}^{(j)}\left(\varepsilon^{\prime}\right)\right) \cup\left(\bigcup_{i \geq 1} A_{i}\left(\varepsilon^{\prime}\right)\right) .
$$

Le lemme 4.3.7, appliqué à cette inclusion, assure l'existence d'un entier $m\left(\varepsilon^{\prime}\right) \in \mathbf{N}$ tel que pour tout $n \geq m\left(\varepsilon^{\prime}\right)$

$$
A_{0}\left(\varepsilon^{\prime}\right) \subset\left(\bigcup_{j \leq n} A_{0}^{(j)}\right) \cup\left(\bigcup_{i \in M} C_{i}\right)
$$

où $M$ est un ensemble fini et les $C_{i}$ sont des cylindres de $\operatorname{Gr}(X)$ tels que $\left.\| \mu_{X}\left(C_{i}\right)\right) \|<\varepsilon^{\prime}$ pour tout $i \in M$. En remarquant que la suite de cylindres de $\operatorname{Gr}(X)$ définie par $B_{0}^{(j)}\left(\varepsilon^{\prime}\right):=A_{0}^{(j)}\left(\varepsilon^{\prime}\right) \cap A_{0}\left(\varepsilon^{\prime}\right)$, par les $A_{i}\left(\varepsilon^{\prime}\right)$ pour tout $i \in I\left(\varepsilon^{\prime}\right)$ et les $A_{i}^{(j)}\left(\varepsilon^{\prime}\right)$ pour tout $i \in I_{j}\left(\varepsilon^{\prime}\right)$, est une $\varepsilon^{\prime}$-approximation cylindrique de $A^{(j)}$ pour tout $j \in \mathbf{N}$, et que, pour tout $0 \leq j \leq n$, l'on peut choisir les $A_{0}^{(j)}\left(\varepsilon^{\prime}\right)$ disjoints deux à deux, on a dans $\widehat{M}_{k}$ l'égalité

$$
\mu_{X}\left(A_{0}\left(\varepsilon^{\prime}\right)\right)=\sum_{j=0}^{n} \mu_{X}\left(B_{0}^{(j)}\left(\varepsilon^{\prime}\right)\right)+\sum_{i \in I} \mu_{X}\left(C_{i}^{\prime}\right)
$$

où les cylindres $C_{i}^{\prime}:=C_{i} \cap A_{0}\left(\varepsilon^{\prime}\right)$ de $\operatorname{Gr}(X)$ sont tels que $\left.\| \mu_{X}\left(C_{i}^{\prime}\right)\right) \|<\varepsilon^{\prime}$. En particulier, ceci entraîne que pour tout $n \geq m\left(\varepsilon^{\prime}\right)$

$$
\mu_{X}\left(A_{0}\left(\varepsilon^{\prime}\right)\right)-\sum_{j=0}^{n} \mu_{X}\left(B_{0}^{(j)}\left(\varepsilon^{\prime}\right)\right) \in F^{q} \widehat{M}_{k}
$$

avec $q=E(-\log (\varepsilon) / \log 2)$ et donc, grâce au choix de $\varepsilon^{\prime}$, que

$$
\mu_{X}(A)-\sum_{j=0}^{n} \mu_{X}\left(B_{0}^{(j)}\left(\varepsilon^{\prime}\right)\right) \in F^{q} \widehat{M}_{k} .
$$

Par ailleurs, par définition de la mesure, il existe $\varepsilon^{\prime}>\varepsilon^{\prime \prime}>0$ tel que

$$
\sum_{j=0}^{n} \mu_{X}\left(B_{0}^{(j)}\left(\varepsilon^{\prime \prime}\right)\right)-\sum_{j=0}^{n} \mu_{X}\left(A^{(j)}\right) \in F^{q} \widehat{M}_{k} .
$$

En outre, par le lemme 6.2.1,

$$
\sum_{j=0}^{n} \mu_{X}\left(B_{0}^{(j)}\left(\varepsilon^{\prime}\right)\right)-\sum_{j=0}^{n} \mu_{X}\left(B_{0}^{(j)}\left(\varepsilon^{\prime \prime}\right)\right) \in F^{q} \widehat{M}_{k}
$$

TOME $132-2004-\mathrm{N}^{\mathrm{O}} 1$ 
En additionnant ces trois dernières relations, on obtient pour tout $n \geq m\left(\varepsilon^{\prime}\right)$,

$$
\left\|\mu_{X}(A)-\sum_{j=0}^{n} \mu_{X}\left(A^{(j)}\right)\right\|<\varepsilon .
$$

THÉORÈme 6.3.5. - 1) Soit $\left(A_{i}\right)_{i \in \mathbf{N}}$ une famille de mesurables deux à deux disjoints. Leur réunion $\bigcup_{i \in \mathbf{N}} A_{i}$ est mesurable si et seulement si la suite des volumes $\left(\mu_{X}\left(A_{i}\right)\right)_{i \in \mathbf{N}}$ tend vers 0 quand $i$ tend vers l'infini. Dans ce cas, on a la relation

$$
\mu_{X}\left(\bigcup_{n \in \mathbf{N}} A_{n}\right)=\sum_{n \in \mathbf{N}} \mu_{X}\left(A_{n}\right) .
$$

2) Soit $\left(A_{n}\right)_{n \in \mathbf{N}}$ une suite d'ensembles mesurables de $\operatorname{Gr}(X)$ vérifiant $A_{n+1} \subset A_{n}$ et posons $A=\bigcap_{n} A_{n}$. Alors les assertions suivantes sont équivalentes :

a) l'ensemble $A$ est mesurable;

b) la suite $\left(\mu_{X}\left(A_{n}\right)\right)$ converge dans $\widehat{M}_{k}$;

c) la suite $\left(\mu_{X}\left(A_{n} \backslash A_{n+1}\right)\right)_{n \in \mathbf{N}}$ tend vers 0 dans $\widehat{M}_{k}$.

Si elles sont vérifiées, on a $\mu_{X}(A)=\lim _{n \rightarrow+\infty} \mu_{X}\left(A_{n}\right)$.

3) Soit $\left(B_{n}\right)_{n \in \mathbf{N}}$ une suite d'ensembles mesurables de $\operatorname{Gr}(X)$ vérifiant $B_{n} \subset$ $B_{n}$ et posons $B=\bigcup_{n} A_{n}$. Alors les assertions suivantes sont équivalentes :

a) l'ensemble $B$ est mesurable;

b) la suite $\left(\mu_{X}\left(B_{n}\right)\right)$ converge dans $\widehat{M}_{k}$;

c) la suite $\left(\mu_{X}\left(B_{n} \backslash B_{n-1}\right)\right)_{n \in \mathbf{N}}$ tend vers 0 dans $\widehat{M}_{k}$. Si elles sont vérifiées, on a $\mu_{X}(B)=\lim _{n \rightarrow+\infty} \mu_{X}\left(B_{n}\right)$.

Démonstration. - Dans cette preuve, on omettra les indices $X$. Le premier point découle de la proposition 6.3 .4 et du fait que la topologie de $\widehat{M}_{k}$ est ultramétrique.

2) Pour tout $n \in \mathbf{N}$, posons $C_{n}=A_{n} \backslash A_{n+1}$. Les $C_{n}$ sont alors des ensembles mesurables disjoints. Par ailleurs,

$$
\bigcup_{n \in \mathbf{N}} C_{n}=A_{0} \backslash\left(\bigcap_{m \geq 0} A_{m}\right) .
$$

Comme la topologie de $\widehat{M}_{k}$ est ultramétrique et complète, la suite $\left(\mu\left(C_{n}\right)\right)$ tend vers 0 dans $\widehat{M}_{k}$ si et seulement si la suite $\left(\mu\left(A_{n}\right)\right)$ est coverge dans $\widehat{M}_{k}$. Dans ce cas, l'assertion $6.3 .5,1)$ assure que $\mu(A)=\lim \mu\left(A_{n}\right)$.

3) Cette assertion découle de la précédente en passant aux complémentaires.

6.4. Les ensembles de mesure nulle. - Soit $\mathbf{D}_{X}^{*}$ la famille des sousensembles $E \subset \operatorname{Gr}(X)$ pour lesquels il existe des ensembles mesurables $A$ et $B$ tels que $A \subset E \subset B$ et $\mu(B \backslash A)=0$. 
Lemme 6.4.1. - Les ensembles $\mathbf{D}_{X}$ et $\mathbf{D}_{X}^{*}$ sont égaux.

Démonstration. - L'inclusion $\mathbf{D}_{X} \subset \mathbf{D}_{X}^{*}$ est claire. Soit $E \in \mathbf{D}_{X}^{*}$. Soit $\varepsilon>0$. Par définition, il existe des ensembles mesurables $A \in \mathbf{D}_{X}$ et $B \in \mathbf{D}_{X}$ tels que $A \subset E \subset B$ et $\mu(B \backslash A)=0$. Il existe un réel $\varepsilon^{\prime}>0$, une $\varepsilon^{\prime}$-approximation cylindrique $\left(A_{0} ;\left(A_{i}\right)_{i \geq 1}\right)$ de $A$ et une $\varepsilon^{\prime}$-approximation cylindrique $\left(B_{0} ;\left(B_{j}\right)_{j \geq 1}\right)$ de $B$ telles que

$$
\varepsilon^{\prime}<\varepsilon \quad \text { et }\left\|\mu\left(B_{0} \backslash A_{0}\right)\right\|<\varepsilon .
$$

La suite de cylindres définie par $E_{0}:=B_{0}$, par $B_{0} \backslash A_{0}$, par les $A_{i}$ et les $B_{i}$ pour tout $i \geq 1$ est une $\varepsilon$-approximation de $E$.

Lemme 6.4.2. - Soit $E \subset \operatorname{Gr}(X)$. Alors $E$ est mesurable de mesure nulle si et seulement si pour tout $\varepsilon>0$, il existe un ensemble au plus dénombrable $I$ et une suite de cylindres $\left(E_{i}\right)_{i \in I}$ telle que $E \subset \bigcup_{i \in I} E_{i}$ et $\left\|\mu\left(E_{i}\right)\right\|<\varepsilon$ pour tout $i \in I$.

Démonstration. - Cette condition est suffisante de manière évidente. Inversement, soit $E$ un ensemble mesurable de mesure nulle. Soit $\varepsilon>0$. Par construction de la mesure, il existe un réel $\varepsilon^{\prime}>0$ et une $\varepsilon^{\prime}$-approximation cylindrique $\left(E_{0}^{\prime} ;\left(E_{i}^{\prime}\right)_{i \geq 1}\right)$ de $E$ tels que

$$
\varepsilon^{\prime}<\varepsilon \quad \text { et }\left\|\mu\left(E_{0}^{\prime}\right)\right\|<\varepsilon \text {. }
$$

La suite définie par $E_{0}:=\varnothing$ et par les $E_{i}^{\prime}$ pour tout $i \in \mathbf{N}$ vérifie la propriété annoncée.

Corollaire 6.4.3. - Soit $A$ un sous-ensemble de $\operatorname{Gr}(X)$, tel que $A \subset C$, avec $C$ un ensemble mesurable de mesure nulle de $\operatorname{Gr}(X)$. Alors $A$ est un ensemble mesurable de mesure nulle.

6.5. La définition de l'intégrale. - On supposera que tous les $\mathbb{D}$-schémas formels sont admissibles, purement de dimension relative $d$. Fixons un tel schéma formel $X$.

DÉFinition 6.5.1. - Soit $A$ un ensemble mesurable de $\operatorname{Gr}(X)$. On dit qu'une application $\psi: A \rightarrow \widehat{M}_{k}$ est mesurable si les fibres de $\psi$ sont mesurables dans $\operatorname{Gr}(X)$. Si la somme $\sum_{\mathbf{a} \in \widehat{M}_{k}} \mu_{X}\left(\psi^{-1}(\mathbf{a})\right) \mathbf{a}$ est convergente, i.e. si l'ensemble des $\mathbf{a} \in \widehat{M}_{k}$ tels que $\mu_{X}\left(\psi^{-1}(\mathbf{a})\right) \neq 0$ est au plus dénombrable et si $\sum \mu_{X}\left(\psi^{-1}(\mathbf{a})\right) \mathbf{a}$ converge dans $\widehat{M}_{k}$, on dit que $\psi$ est intégrable sur $A$. On définit alors l'intégrale motivique de $\psi$ par

$$
\int_{A} \psi \mathrm{d} \mu_{X}:=\sum_{i \in \mathbf{N}} \mu_{X}\left(\psi^{-1}(\mathbf{a})\right) \mathbf{a} .
$$

TOME $132-2004-\mathrm{N}^{\mathrm{O}} 1$ 
DÉfinition 6.5.2. - Pour tout ensemble mesurable $A$ et toute application $\alpha: A \rightarrow \mathbf{Z} \cup\{\infty\}$, on dit que $\alpha$ est mesurable si $\alpha^{-1}(n) \subset A$ est mesurable, pour tout $n \in \mathbf{Z}$, et exponentiellement intégrable si $\mathbf{L}^{-\alpha}$ est intégrable, i.e. si les fibres finies de $\alpha$ sont mesurables et si la somme $\sum_{n \in \mathbf{Z}} \mu_{X}\left(\alpha^{-1}(n)\right) \mathbf{L}^{-n}$ converge dans $\widehat{M}_{k}$.

Lemme 6.5.3. - Soient $A$ et $B$ deux parties mesurables de $\operatorname{Gr}(X)$ telles que $A \subset B$. Si $\psi: B \rightarrow \widehat{M}_{k}$ est une application intégrable sur $B$, alors sa restriction $\grave{a} A, \psi_{\mid A}: A \rightarrow \widehat{M}_{k}$ est intégrable sur $A$.

Démonstration. - L'ensemble des a $\in \widehat{M}_{k}$ tels que $\mu_{X}\left(\left(\psi_{\mid A}\right)^{-1}(\mathbf{a})\right)$ est non nul est au plus dénombrable. Soient $\left(\mathbf{a}_{n}\right)_{n \in \mathbf{N}}$ ces éléments. Comme $\widehat{M}_{k}$ est complet et ultramétrique, la série $\sum_{i \in \mathbf{N}}\left(\psi^{-1}\left(\mathbf{a}_{n}\right) \cap A\right) \mathbf{a}_{n}$ converge si et seulement si son terme général tend vers 0 . Soit $\varepsilon>0$, il existe $N \in \mathbf{N}$ tel que pour tout $n \geq N,\left\|\mu_{X}\left(\psi^{-1}\left(\mathbf{a}_{n}\right) \mathbf{a}_{n}\right)\right\|<\varepsilon$. L'inclusion de l'énoncé entraîne pour ces mêmes indices, que $\left\|\mu_{X}\left(\left(\psi_{\mid A}\right)^{-1}\left(\mathbf{a}_{n}\right) \mathbf{a}_{n}\right)\right\|<\varepsilon$. D'où le résultat.

Lemme 6.5.4. - Soient $A$ et $B$ deux parties mesurables. Soit $\psi$ une application intégrable sur $A \cup B$, alors on a la relation

$$
\int_{A \cup B} \psi \mathrm{d} \mu_{X}=\int_{A} \psi \mathrm{d} \mu_{X}+\int_{B} \psi \mathrm{d} \mu_{X}-\int_{A \cap B} \psi \mathrm{d} \mu_{X}
$$

Démonstration. - Par définition, on a

$$
\int_{A \cup B} \psi \mathrm{d} \mu_{X}=\sum_{i \in \mathbf{N}} \mu_{X}\left(\psi^{-1}\left(\mathbf{a}_{i}\right)\right) \mathbf{a}_{i} .
$$

Le résultat vient alors du fait que

$\mu_{X}\left(\psi^{-1}\left(\mathbf{a}_{i}\right)\right)=\mu_{X}\left(\psi^{-1}\left(\mathbf{a}_{i}\right) \cap A\right)+\mu_{X}\left(\psi^{-1}\left(\mathbf{a}_{i}\right) \cap B\right)-\mu_{X}\left(\psi^{-1}\left(\mathbf{a}_{i}\right) \cap(A \cap B)\right)$.

Autrement dit,

$$
\mu_{X}\left(\psi^{-1}\left(\mathbf{a}_{i}\right)\right)=\mu_{X}\left(\left(\psi_{\mid A}\right)^{-1}\left(\mathbf{a}_{i}\right)\right)+\mu_{X}\left(\left(\psi_{\mid B}\right)^{-1}\left(\mathbf{a}_{i}\right)\right)-\mu_{X}\left(\left(\psi_{\mid A \cap B}\right)^{-1}\left(\mathbf{a}_{i}\right)\right) .
$$

L'assertion découle donc de la définition de l'intégrale.

LEMME 6.5.5. - Soit A un ensemble mesurable de $\operatorname{Gr}(X)$. Soit B un ensemble de mesure nulle. Soit $\psi$ une application intégrable sur $A$. On a alors la relation

$$
\int_{A} \psi \mathrm{d} \mu=\int_{A \backslash B} \psi \mathrm{d} \mu .
$$

Démonstration. - La preuve ressemble à la précédente. Il suffit de remarquer que $\mu\left(\psi^{-1}\left(\mathbf{a}_{i}\right)\right)=\mu\left(\psi^{-1}\left(\mathbf{a}_{i}\right) \cap B\right)+\mu\left(\psi^{-1}\left(\mathbf{a}_{i}\right) \backslash B\right)$. Or $\psi^{-1}\left(\mathbf{a}_{i}\right) \cap B$ est contenu dans $B$, donc de mesure nulle. Donc $\mu\left(\psi^{-1}\left(\mathbf{a}_{i}\right)\right)=\mu\left(\psi^{-1}\left(\mathbf{a}_{i}\right) \backslash B\right)$. 


\section{Mesurabilité par image directe et image inverse}

7.1. Une estimation du volume des fibres d'un morphisme. — Soient $Y$ et $X$ deux $\mathbb{D}$-schémas formels admissibles, purement de dimension relative $d$. Soit $h: Y \rightarrow X$ un $\mathbb{D}$-morphisme de schémas formels. Les deux lemmes suivants permettent, sous certaines conditions, d'estimer le volume de l'image inverse par $h$ d'un cylindre en fonction de son propre volume. Le lemme 7.1.3 va jouer un rôle essentiel dans la preuve du théorème de changement de variables.

Lemme 7.1.1. - Supposons $Y \rightarrow \mathbb{D}$ lisse. Alors pour tout $n \geq \max \left(e, c_{X} e^{\prime}\right)$, pour tout $z \in \Delta_{e, e^{\prime}}$ et tout $x \in \operatorname{Gr}(X)$, tel que $\pi_{n, X}(h(z))=\pi_{n, X}(x)$, il existe $y \in \operatorname{Gr}(Y)$ tel que $h(y)=x$ et $\pi_{n-e, Y}(z)=\pi_{n-e, Y}(y)$.

Démonstration. - Commençons par remarquer que $h(z) \in \operatorname{Gr}^{\left(e^{\prime}\right)}(Y)$ implique que $x \in \operatorname{Gr}^{\left(e^{\prime}\right)}(X)$.

Réduction géométrique. - Soit $z \in \operatorname{Gr}(Y)$ de corps résiduel $k^{\prime}$ tel que $\varphi_{z} \in$ $\Delta_{e, e^{\prime}}\left(k^{\prime}\right)$ et $x \in \operatorname{Gr}(X)$. Quitte à grossir $k^{\prime}$, on peut supposer que $k^{\prime}$ est également le corps résiduel de $x$. La question est locale en $X$, on peut donc supposer que $X$ est affine. La question étant locale en $Y$, on peut supposer que $Y$ est affine et comme $Y \rightarrow \mathbb{D}$ est supposé lisse, on peut supposer qu'il existe un $\mathbb{D}$ morphisme étale $Y \rightarrow \mathbb{B}_{R}^{d}$. On peut supposer que $Y=\mathbb{B}_{R}^{d}$. En effet, le lemme 3.3.6 assure qu'au-dessus de tout point la fibre du $k$-morphisme de schémas $\operatorname{Gr}(Y) \rightarrow Y_{0}$ est isomorphe à $\operatorname{Gr}\left(\mathbb{B}_{R}^{d}\right)$.

Premier cas : supposons que $X=\mathbb{B}_{R}^{d}$. - Il nous suffit de prouver que pour tout $v \in\left(R_{k^{\prime}}\right)^{d}$, il existe $u \in\left(R_{k^{\prime}}\right)^{d}$ vérifiant l'équation

$$
h\left(\varphi_{z}+\pi^{n+1-e} u\right)=h\left(\varphi_{z}\right)+\pi^{n+1} v
$$

Ce système est alors équivalent à

$$
\pi^{-e} J_{h}\left(\varphi_{z}\right) \cdot u+\pi(\cdots)=v
$$

où $J_{h}$ désigne la matrice jacobienne de $h$. Soit $M$ la matrice adjointe de $J_{h}$. On est ramené à résoudre le système

$$
\pi^{-e} M\left(\varphi_{z}\right) J_{h}\left(\varphi_{z}\right) \cdot u+\pi(\cdots)=M\left(\varphi_{z}\right) v
$$

Par définition de la fonction $\operatorname{ord}_{\pi}(\mathrm{Jac})_{h}$ et comme $Y=X=\mathbb{B}_{R}^{d}$, le déterminant de $J_{h}\left(\varphi_{z}\right)$ appartient à $\pi^{e}$. Par conséquent, la réduction modulo l'idéal maximal du système 6 précédent est linéaire sur le corps $k^{\prime}$, de déterminant non nul. Il admet donc une solution par le lemme de Hensel (cf. [9], chap. III, $\S 4, \mathrm{n}^{\circ} 6$, cor. 2).

TOME $132-2004-\mathrm{N}^{\mathrm{O}} 1$ 
Deuxième cas : $X=\operatorname{Spf} R\left\{x_{1}, \ldots, x_{N}\right\} / I$. - Notons $\widetilde{h}$ le $\mathbb{D}$-morphisme composé

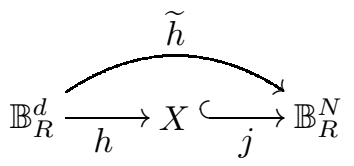

où $j$ est le morphisme d'immersion. La question étant locale en $X$, on peut supposer, grâce au lemme 4.5.3, que $X$ est d'intersection complète, i.e. qu'il existe $N-d$ éléments $\left(f_{i}\right)_{1 \leq i \leq N-d}$ de $I$ qui l'engendrent, qu'il existe un entier $e^{\prime \prime} \leq c_{X} e^{\prime}$ et un mineur $\delta$ d'ordre $N-d$ de la matrice jacobienne $\Delta$ des $f_{i}$ tels que $\delta\left(\widetilde{h}\left(\varphi_{z}\right)\right) \in\left(\pi^{e^{\prime \prime}}\right)$ et que, pour tout autre mineur $\delta^{\prime}$ d'ordre $N-d$, $\delta^{\prime}\left(\widetilde{h}\left(\varphi_{z}\right)\right) \in\left(\pi^{\nu}\right)$ avec $\nu \geq e^{\prime \prime}$. Quitte à réordonner les $X_{i}$, on peut supposer que $\delta$ est calculé à partir des $N-d$ premières colonnes de $\Delta$. Comme précédemment, pour prouver l'assertion, il nous suffit de montrer que pour tout $v \in\left(R_{k^{\prime}}\right)^{N}$ tel que $f_{j}\left(h\left(\varphi_{z}\right)+\pi^{n+1} v\right)=0$, pour tout $1 \leq j \leq N-d$, il existe $u \in\left(R_{k^{\prime}}\right)^{d}$ tel que

$$
h\left(\varphi_{z}+\pi^{n+1-e} u\right)=h\left(\varphi_{z}\right)+\pi^{n+1} v .
$$

Soit $v \in\left(R_{k^{\prime}}\right)^{N}$. On peut se ramener au cas où $X=\mathbb{B}_{R}^{d}$. En effet, le $\mathbb{D}$ morphisme composé

$$
\mathbb{B}_{R}^{d} \stackrel{\widetilde{h}}{\longrightarrow} \mathbb{B}_{R}^{N} \longrightarrow \mathbb{B}_{R}^{N-d}
$$

défini par

$$
f_{i}\left(h_{1}\left(x_{1}, \ldots, x_{d}\right), \ldots, h_{N}\left(x_{1}, \ldots, x_{d}\right)\right) \longleftrightarrow f_{i}\left(z_{1}, \ldots, z_{N}\right) \longleftrightarrow y_{i}
$$

est constant d'image 0 (par définition de $X$ ). En particulier, ceci entraîne que le produit des matrices jacobiennes $\Delta\left(\widetilde{h}\left(\varphi_{z}\right)\right) \cdot J_{h}\left(\varphi_{z}\right)$ est nul et les colonnes de la matrice $J_{h}\left(\varphi_{z}\right)$ appartiennent au noyau de la matrice $\Delta\left(\widetilde{h}\left(\varphi_{z}\right)\right)$, i.e. les coordonnées de chacune de ces colonnes sont solutions du système

$$
\pi^{-e^{\prime \prime}} M^{\prime}\left(\widetilde{h}\left(\varphi_{z}\right)\right) \cdot \Delta\left(\widetilde{h}\left(\varphi_{z}\right)\right) w=0,
$$

où $M^{\prime}$ est la matrice $(N-d) \times(N-d)$ définie comme la matrice adjointe de la sous-matrice $(N-d) \times(N-d)$ de $\Delta$ formée des $N-d$ premières colonnes et $w \in\left(R_{k^{\prime}}\right)^{N}$. Par hypothèse, les équations définissant (8) expriment donc les $N-d$ premières coordonnées de $w$ en fonction des $d$ dernières. On note $\bar{w} \in\left(k^{\prime}\right)^{N}$ l'image de $w$ dans la somme des quotients de $R_{k^{\prime}}$ modulo l'idéal maximal. Le lemme de Hensel ( $c f$. [9, chap. III, $\S 4, \mathrm{n}^{\mathrm{o}} 6$, cor. 2]) assure que $w \in\left(R_{k^{\prime}}\right)^{N}$ est solution du système (8) si et seulement si

$$
\pi^{-e^{\prime \prime}} M^{\prime}\left(\widetilde{h}\left(\varphi_{z}\right)\right) \cdot \Delta\left(\widetilde{h}\left(\varphi_{z}\right)\right) \bar{w}=0 \in\left(k^{\prime}\right)^{N} .
$$

Comme $f_{j}\left(h\left(\varphi_{z}\right)+\pi^{n+1} v\right)=0$ pour tout $1 \leq j \leq N-d, \bar{v}$ est solution du système (9) et donc $v$ est solution du système (8). Ceci implique, en particulier,

BULletin DE LA SOCiÉtÉ MATHÉMATIQUE DE FRANCE 
que les $d$ premières coordonées de $v$ s'expriment en fonction des $N-d$ dernières, et ces relations (linéaires) sont celles induites par le système (8).

Le système (7) étant équivalent à

$$
\pi^{-e} J_{h}\left(\varphi_{z}\right) \cdot u+\pi(\cdots)=v
$$

il suffit donc de résoudre, puisque les coordonnées du vecteur $J_{h}\left(\varphi_{z}\right) \cdot u$ vérifie les mêmes relations que celles de $v$, le système linéaire suivant :

$$
\pi^{-e} \widetilde{J}_{h}\left(\varphi_{z}\right) \cdot\left(\begin{array}{c}
u_{N-d+1} \\
u_{N-d+2} \\
\vdots \\
u_{N}
\end{array}\right)+\pi(\cdots)=\left(\begin{array}{c}
v_{N-d+1} \\
v_{N-d+2} \\
\vdots \\
v_{N}
\end{array}\right)
$$

où $\widetilde{J}_{h}$ est la sous-matrice de $J_{h}$ formée des $d$ dernières lignes. Soient $x_{N-d+1}, \ldots, x_{N}$ les $d$ dernières coordonnées parmi $x_{1}, \ldots, x_{N}$. Ces coordonnées définissent un $\mathbb{D}$-morphisme de schémas formels

$$
p: X \longrightarrow \mathbb{B}_{R}^{d} \text {. }
$$

Par construction, la matrice jacobienne du morphisme $p \circ h$ n'est autre que la matrice $\widetilde{J}_{h}$. En outre, les hypothèses assurent que l'image de la base $\left(h \circ \varphi_{z}\right)^{*} \mathrm{~d} x_{i_{N-d+1}}, \ldots,\left(h \circ \varphi_{z}\right)^{*} \mathrm{~d} x_{i_{N}}$ par le morphisme

$$
\left(h \circ \varphi_{z}\right)^{*} p^{*} \Omega_{\mathbb{B}_{R}^{d} / \mathbb{D}}^{1} \longrightarrow\left(h \circ \varphi_{z}\right)^{*} \Omega_{X / \mathbb{D}}^{1} /(\text { torsion })
$$

définit une base de $\left(h \circ \varphi_{z}\right)^{*} \Omega_{X / \mathbb{D}}^{1} /($ torsion $)$. Autrement dit,

$$
\operatorname{ord}_{\pi}(\operatorname{Jac})_{p}\left(h\left(\varphi_{z}\right)\right)=0 .
$$

La formule 5.1.7 implique que $\operatorname{ord}_{\pi}(\mathrm{Jac})_{p \circ h}\left(\varphi_{z}\right)=e$. On est donc ramené au premier cas en considérant le $\mathbb{D}$-morphisme $p \circ h: \mathbb{B}_{R}^{d} \rightarrow \mathbb{B}_{R}^{d}$.

LEMME 7.1.2. - Soit $k^{\prime} \supset k$ une extension de corps contenant $k$. Soit $M$ une matrice carrée d'ordre d à coefficients dans $R_{k^{\prime}}$. Le système linéaire $M \cdot X=0$ définit un sous-D-schéma formel fermé de $Z \hookrightarrow \mathbb{B}_{R}^{d}$. Si $j \in \mathbf{N}$, les ensembles constructibles $\operatorname{Gr}_{j}(Z)$ de $\operatorname{Gr}_{j}\left(\mathbb{B}_{R}^{d}\right)$ définissent une suite d'applications $\psi_{j}$

$$
\ldots \stackrel{\psi_{n+1}}{\longrightarrow} \operatorname{Gr}_{n}(Z) \stackrel{\psi_{n}}{\longrightarrow} \operatorname{Gr}_{n-1}(Z) \stackrel{\psi_{n-1}}{\longrightarrow} \cdots \stackrel{\psi_{1}}{\longrightarrow} \operatorname{Gr}_{0}(Z)
$$

dont les fibres sont, pour tout $j \geq 1$, des espaces affines.

Démonstration. — De tels fibres s'identifient aux solutions en $u \in\left(R_{k^{\prime}}\right)^{d}$, pour $v \in\left(R_{k^{\prime}}\right)^{d}$ fixé, du système linéaire

$$
\pi^{j} M \cdot u \equiv \pi^{j} \cdot v \quad \bmod \left(\pi^{j+1}\right) .
$$

Ce système équivaut à $M \cdot u \equiv v \bmod (\pi)$. Ces équations induisent donc des équations linéaires en les premières composantes dans $R_{k^{\prime}}$ de chaque coordonnée $u_{i}$, pour $1 \leq i \leq d$. Le résultat en découle.

TOME $132-2004-\mathrm{N}^{\mathrm{O}} 1$ 
Lemme 7.1.3. - Supposons que $Y \rightarrow \mathbb{D}$ est lisse. Soient $B \subset \operatorname{Gr}(Y)$ un $m$ cylindre et $A=h(B)$. Supposons que $\operatorname{ord}_{\pi}(\mathrm{Jac})_{h}(\varphi)$ est constant de valeur $e<\infty$ pour tout $\varphi \in B$, et $A \subset \mathrm{Gr}^{\left(e^{\prime}\right)}(X)$, avec $e^{\prime} \in \mathbf{N}$. Alors $A$ est un cylindre. De plus, si la restriction de $h$ à $B$ est injective, alors, pour tout entier $n \geq \max \left(2 e+c_{X}, m+e\right)$ :

1) si $\varphi$ et $\varphi^{\prime}$ appartiennent $\grave{a} B$ et $\pi_{n, X}(h(\varphi))=\pi_{n, X}\left(h\left(\varphi^{\prime}\right)\right)$, alors on a $\pi_{n-e, Y}(\varphi)=\pi_{n-e, Y}\left(\varphi^{\prime}\right)$;

2) dans $M_{k}$, on a la relation

$$
\left[\pi_{n, Y}(B)\right]=\left[\pi_{n, X}(A)\right] \mathbf{L}^{e} .
$$

Démonstration. - 1) Prouvons tout d'abord que $A:=h(B)$ est un cylindre. Par hypothèse, $B$ est contenu dans $\Delta_{e, e^{\prime}}$. Soit $n \geq 2 e+c_{X} e^{\prime}$ un entier tel que $B$ soit un cylindre de rang $n-e$. Soit $x \in \pi_{n, X}^{-1}\left(\pi_{n, X}(A)\right)$. Il existe donc $b \in B$ tel que

$$
\pi_{n, X}(x)=\pi_{n, X}(h(b)) .
$$

Le lemme 7.1.1 assure alors qu'il existe $y \in \operatorname{Gr}(Y)$ tel que

$$
\text { (i) } h(y)=x \text { et (ii) } \pi_{n-e, Y}(b)=\pi_{n-e, Y}(y) \text {. }
$$

Comme $B$ est un cylindre de rang $n-e$, la condition (ii) entraîne que $y \in B$. La condition (i) assure que $x \in A$. On a donc montré que $A=\pi_{n, X}^{-1}\left(\pi_{n, X}(A)\right)$. Par ailleurs, la relation $\pi_{n, X}(A)=h_{n}\left(\pi_{n, Y}(B)\right)$ implique que cet ensemble est constructible.

2) Prouvons l'assertion 7.1.3, 1). Soit $n \in \mathbf{N}$ tel que $B$ soit un cylindre de rang $n-e$. Soient $\varphi$ et $\varphi^{\prime}$ deux points de $B \subset \Delta_{e, e^{\prime}}$ tels que $\pi_{n, X}(h(\varphi))=$ $\pi_{n, X}\left(h\left(\varphi^{\prime}\right)\right)$. Le lemme 7.1.1, appliqué à $\varphi^{\prime}$ et $\varphi$, assure l'existence de $y \in B$ tel que $y=h\left(\varphi^{\prime}\right)$ et $\pi_{n-e, Y}(\varphi)=\pi_{n-e, Y}(y)$. Par injectivité de $h, y=\phi^{\prime}$ et donc $\pi_{n-e, Y}(\varphi)=\pi_{n-e, Y}\left(\varphi^{\prime}\right)$.

3) Passons à l'assertion 7.1.3, 2). Nous allons distinguer les cas d'égales et d'inégales caractéristiques.

Le cas d'égales caractéristiques. - Nous allons montrer l'énoncé plus précis suivant : l'application $h_{n}$ est une fibration localement triviale par morceaux audessus de $\pi_{n, X}(A)$. Il nous suffit de démontrer le résultat pour le cylindre $\Delta_{e, e^{\prime}}$. L'assertion 1) implique que la fibre de $h_{n}$ au-dessus de $\pi_{n, X}(h(\varphi))$ peut se décrire comme

$$
\left\{\varphi+\pi^{n+1-e} u \bmod \pi^{n+1} \mid\left(J_{h}(\varphi) \cdot u\right) \equiv 0 \bmod \pi^{n+1}\right\} .
$$

Le théorème 4.2.3 assure qu'il nous suffit de vérifier que les fibres sont des espaces affines de dimension $e$. La question étant locale en $X$ et $Y$, on peut supposer que $X$ et $Y$ sont affines. Supposons que $X$ est défini dans $\mathbb{B}_{R}^{N}$ par l'idéal $I$, engendré par $m$ éléments $\left(f_{i}\right)_{1 \leq i \leq m}$. Comme le cylindre $h\left(\Delta_{e, e^{\prime}}\right)$ est 
contenu dans $\mathrm{Gr}^{\left(e^{\prime}\right)}(X)$, le lemme 4.5.3 assure qu'il est recouvert par un nombre fini de cylindres $C$, vérifiant la propriété

$$
\operatorname{Gr}(X) \cap C=\operatorname{Gr}\left(R\left\{X_{1}, \ldots, X_{N}\right\} /\left(f_{i_{1}}, \ldots, f_{i_{N-d}}\right)\right) \cap C
$$

Ainsi, on peut supposer que $X \hookrightarrow \mathbb{B}_{R}^{N}$ est d'intersection complète. Soit $\Delta$ la matrice jacobienne $\left(\partial f_{j} / \partial x_{i}\right)_{1 \leq i \leq N, 1 \leq j \leq N-d}$. Quitte à restreindre $C(c f .4 .5 .3,3)$ et éventuellement à réordonner les $X_{j}$, on peut supposer que, pour tout $x \in C$, le mineur d'ordre $N-d$ de $\Delta\left(\varphi_{x}\right)$ formé des $N-d$ premières colonnes de $\Delta\left(\varphi_{x}\right)$ est de valuation $e^{\prime \prime} \leq c_{X} e$ et que les autres mineurs d'ordre $N-d$ de cette matrice sont de valuation supérieure ( $c f$. lemme 4.5.4). Il nous suffit donc de prouver le résultat pour $\Delta_{e, e^{\prime}} \cap h^{-1}(C)$. Soit $x \in \Delta_{e, e^{\prime}} \cap h^{-1}(C)$. Soit $\varphi:$ Spf $R_{k^{\prime}} \rightarrow X$ le $\mathbb{D}$-morphisme de schémas formels correspondant à $x$. Supposons $n \geq 2 e$ et $n \geq e+c_{X} e^{\prime}$. Comme $Y$ est supposé lisse, on peut supposer qu'il existe un $\mathbb{D}$-morphisme étale de schémas formels $Y \rightarrow \mathbb{B}_{R}^{d}$ et, grâce au lemme 3.3.6, que $Y=\mathbb{B}_{R}^{d}$ ( $c f$. preuve du lemme 7.1.1). Par ailleurs, l'étude faite dans la preuve du lemme 7.1.1 assure que l'on peut également supposer que $X=\mathbb{B}_{R}^{d}$. Comme $R_{k^{\prime}}$ est un anneau de valuation discrète, la matrice $J_{h}(\varphi)$ est équivalente à une matrice diagonale, dont les termes diagonaux sont $\pi^{e_{1}}, \ldots, \pi^{e_{d}}$ avec $\sum e_{j}=e$. On est donc ramené à étudier les congruences

$$
\pi^{n+1-e+e_{i}} u_{i} \equiv 0 \bmod \left(\pi^{n+1}\right) .
$$

Ceci entraîne en particulier que $u_{i} \in \pi^{e-e_{i}}$ pour tout $1 \leq i \leq d$. Or, la donnée d'un élément de la fibre correspond à celle des $e$ premiers coefficients de chaque $u_{i}$. Autrement dit, un élément de la fibre correspond à $\sum e_{i}=e$ éléments de $\kappa(x):=k^{\prime}$. Cette description implique que la fibre de $h_{n}$ au-dessus de $\pi_{n, X}(h(\varphi))$ est $k^{\prime}$-isomorphe à $\mathbf{A}_{k^{\prime}}^{e}$.

Le cas d'inégales caractéristiques. — L'additivité de [ ] permet de supposer que $X$ et $Y$ sont affines. Comme $Y$ est lisse, le lemme 3.3.6, qui consiste simplement à choisir un système de coordonnées locales, permet de supposer que $Y=\mathbb{B}_{R}^{d}$. Grâce au lemme 4.5.3 et à l'additivité de [], il nous suffit de démontrer le résultat pour $X$ d'intersection complète, défini dans $\mathbf{B}_{R}^{N}$ par $f_{j}=0$, pour tout $1 \leq j \leq N-d$, et pour un cylindre $A$ sur lequel le mineur d'ordre $N-d$ calculé sur les $N-d$ premières est d'ordre minimal, égal à $e^{\prime \prime} \geq c_{X} e^{\prime}$, parmi les autres mineurs d'ordre $N-d$. Dans ce cas, le raisonnement du lemme 7.1.1 assure que l'on peut supposer que $X=\mathbb{B}_{R}^{d}$.

Comme dans le cas d'égales caractéristiques, il résulte de l'assertion 7.1.3, 1) que l'on peut identifier la fibre de $h_{n}$ au-dessus de $\pi_{n, X}(h(\varphi))$, pour $\varphi \in\left(R_{k^{\prime}}\right)^{d}$ et $\varphi \in B$, à l'ensemble

$$
\left\{\varphi+\pi^{n+1-e} u \bmod \pi^{n+1} \mid\left(J_{h}(\varphi) \cdot u\right) \equiv 0 \quad \bmod \pi^{n+1}\right\} .
$$

Cette description des fibres entraîne que celles-ci peuvent encore s'identifier à

$$
\left\{u \bmod \pi^{e} \mid J_{h}(\varphi) \cdot u \equiv 0 \bmod \pi^{e}\right\} .
$$

TOME $132-2004-\mathrm{N}^{\mathrm{O}} 1$ 
Dans ce cas nous allons montrer que l'application $\pi_{n, Y}(B) \rightarrow \pi_{n, X}(A)$ se factorise par une suite de fibrations localement triviales par morceaux

$$
C_{i, \varphi}:=\left\{u \bmod \pi^{i} \mid J_{h}(\varphi) u \equiv 0 \bmod \pi^{i}\right\} .
$$

Les $C_{i, \varphi}$ sont clairement des ensembles constructibles de $\operatorname{Gr}_{i}\left(\mathbb{B}_{R}^{d}\right)$ pour tout $1 \leq i \leq e$. En outre, pour tout $2 \leq i \leq e$, il existe une application $\psi_{i, \varphi}$ : $C_{i, \varphi} \rightarrow C_{i-1, \varphi}$ induite par le $k$-morphisme de schémas $\theta_{i-1}^{i}$. Il découle du lemme 7.1.2 que les fibres des $\psi_{i, \varphi}$ sont des espaces affines. Nous désignons par $\alpha_{i}(\varphi)$ la fonction qui à $u \in C_{i-1, \varphi}$ associe la dimension de la fibre de $\psi_{i, \varphi}$ audessus de $u$. En outre, $C_{1, \varphi}$ est un espace affine. On note $\alpha_{1}(\varphi)$ sa dimension. Soit $k_{p}^{\prime} \supset k^{\prime}$ une clôture parfaite de $k^{\prime}$. Dans ce cas, $R_{k_{p}^{\prime}}$ est un anneau de valuation discrète. Le raisonnement tenu en égales caractéristiques permet donc d'identifier $C_{e, \varphi} \otimes_{k^{\prime}} k_{p}^{\prime}$ à $\mathbf{A}_{k_{p}^{\prime}}^{e}$. Ceci entraîne en particulier que $\sum \alpha_{i}(\varphi)=e$.

Pour $1 \leq i \leq e$, considérons maintenant l'ensemble

$$
C_{i}=\left\{\left(\pi_{n, Y}(\varphi), \pi_{i, Y}(u)\right) \mid J_{h}(\varphi) \cdot u \equiv 0 \bmod \pi^{i}\right\} .
$$

Les $C_{i}$ sont encore des ensembles constructibles de $\operatorname{Gr}_{n}\left(\mathbb{B}_{R}^{d}\right) \times{ }_{k} \mathrm{Gr}_{i}\left(\mathbb{B}_{R}^{d}\right)$. Les applications $\psi_{i}: C_{i} \rightarrow C_{i-1}$ sont induites par $\theta_{i-1}^{i}$, pour $2 \leq i \leq e$. Par définition, si $h(\varphi) \in A$ est fixé, la restriction de $\psi_{i}$ à l'ensemble

$$
\left\{(\varphi, u) \mid J_{h}(u) \equiv 0 \bmod \pi^{i}\right\}
$$

est égale à $\psi_{i, \varphi}$. On a également une application $\psi_{1}: C_{1} \rightarrow \pi_{n, X}(A)$ induite par la projection sur les premières coordonnées et le morphisme $h_{n}$. Enfin, l'ensemble $C_{e}$ s'identifie à $\pi_{n, Y}(B)$. On a donc le diagramme commutatif suivant :

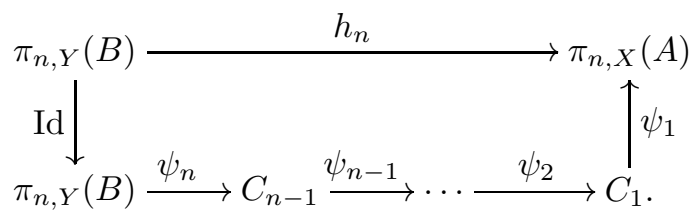

La condition $\sum \alpha_{i}(\varphi)=e$, pour tout $\varphi \in B$, entraîne en particulier que les dimensions des fibres des $\psi_{i, \varphi}$ sont des fonctions constantes. Grâce au lemme 4.2.3, les $\psi_{i}$ sont des fibrations localement triviales et l'on a dans $M_{k}$

$$
\left[\pi_{n, Y}(B)\right]=\left[\pi_{n, X}(A)\right] \mathbf{L}^{e} .
$$

7.2. La mesurabilité de l'image directe. — Dans cette section, nous allons étudier le problème de la stabilité de la propriété de mesurabilité par image directe via un morphisme $h$. Soit $h: Y \rightarrow X$ un $\mathbb{D}$-morphisme de schémas formels admissibles, tous deux purement dedimension relative $d$. Soit $B$ un ensemble mesurable de $\operatorname{Gr}(Y)$. Rappelons que l'on désigne par

$$
\Delta_{e, e^{\prime}}=h^{-1}\left(\operatorname{Gr}^{\left(e^{\prime}\right)}(X)\right) \cap \operatorname{ord}_{\pi}\left(\mathrm{Jac}_{h}^{-1}(e) .\right.
$$

BULletin DE LA SOCiÉtÉ MATHÉMATIQUE DE FRANCE 
Dans ce paragraphe, nous étudions donc le problème de la mesurabilité dans $\operatorname{Gr}(X)$ de $h(B)$. Remarquons d'ores-et-déjà une première difficulté : en général, l'image par $h$ d'un cylindre de $\operatorname{Gr}(Y)$ n'est pas un cylindre de $\operatorname{Gr}(X)$.

LEMme 7.2.1. - Supposons que $Y \rightarrow \mathbb{D}$ est lisse. Si B est un cylindre de $\operatorname{Gr}(Y)$, alors on a les propriétés suivantes :

1) Il existe un cylindre $C$ de $\operatorname{Gr}(X)$ tel que $h(B) \subset C$ et

$$
\left\|\mu_{X}(C)\right\| \leq\left\|\mu_{Y}(B)\right\| \text {. }
$$

2) Si $B \cap \Sigma_{h}=\varnothing$, alors $h(B)$ est un cylindre.

Démonstration. - Supposons d'abord que $\left\|\mu_{Y}(B)\right\|=0$. Comme $B$ est un cylindre et que $Y$ est lisse, ceci entraîne que $B=\varnothing$ et que $h(B)=\varnothing$. Supposons désormais que $\left\|\mu_{Y}(B)\right\| \neq 0$. Comme $\operatorname{Gr}\left(X_{\text {sing }}\right)$ est contenu dans un cylindre de volume aussi petit que l'on veut, on peut supposer, pour alléger les notations, que $h(B) \subset \operatorname{Gr}(X) \backslash \operatorname{Gr}\left(X_{\text {sing }}\right)$. Posons

$$
h^{(e)}(B):=h\left(h^{-1}\left(\operatorname{Gr}^{(e)}(X)\right) \cap B\right) .
$$

Cet ensemble est un cylindre par le lemme 7.1.3. Par hypothèse,

$$
h(B)=\bigcup_{e \in \mathbf{N}} h^{(e)}(B) .
$$

La quasi-compacité de la topologie constructible ( $c f$. lemme 4.3.7) et le fait que, si $e^{\prime} \geq e, \operatorname{Gr}^{\left(e^{\prime}\right)}(X) \supset \operatorname{Gr}^{(e)}(X)$, impliquent qu'il existe $e_{0} \in \mathbf{N}$ tel que $h(B)=h^{\left(e_{0}\right)}(B)$. Notons $r_{B}$ le rang du cylindre $B$. Pour $n \geq \max \left(r_{B}, c_{X} e\right)$, l'ensemble

$$
A_{n}^{(e)}:=\pi_{n, X}^{-1}\left(\pi_{n, X}\left(h^{(e)}(B)\right)\right)
$$

est un $n$-cylindre de $\operatorname{Gr}(X)$, contenant $h^{(e)}(B)$ et contenu dans $\operatorname{Gr}^{(e)}(X)$ et qui est stable de rang $n$. Soit $n \geq \max \left(r_{B}, c_{X} e\right)$. Par suite,

$$
\mu_{X}\left(A_{n}^{(e)}\right)=\left[\pi_{n, X}\left(h^{(e)}(B)\right)\right] \mathbf{L}^{-(n+1) d}, \quad\left\|\mu_{X}\left(A_{n}^{(e)}\right)\right\| \leq 2^{-(n+1) d+\operatorname{dim} \pi_{n, Y}(B)} .
$$

Or $\left\|\mu_{Y}(B)\right\|=2^{-(n+1) d+\operatorname{dim} \pi_{n, Y}(B)}$; la première assertion en découle. Le lemme 4.3.7 et l'hypothèse $B \cap \Sigma_{h}=\varnothing$ entraînent qu'il existe un entier $e^{\prime}$ tel que $B$ est contenu dans $h^{-1}\left(\mathrm{Gr}^{\left(e^{\prime}\right)}(X)\right)$ et que l'on peut supposer que $\operatorname{ord}_{\pi}(\mathrm{Jac})_{h}$ est borné sur $B$. La seconde assertion découle alors du lemme clé 7.1.3.

Corollaire 7.2.2. - Supposons que $Y \rightarrow \mathbb{D}$ est lisse. Soient $B \subset \operatorname{Gr}(Y)$ et $A \subset \operatorname{Gr}(X)$ des ensembles fortement mesurables tels que $B \cap \Sigma_{h}=\varnothing$ et $h(B)=A$. Alors, pour tout $\varepsilon>0$, il existe une $\varepsilon$-approximation cylindrique $\left(B_{j}\right)_{j \in \mathbf{N}}$ de $B$ et une $\varepsilon$-approximation cylindrique $\left(A_{i}\right)_{i \in \mathbf{N}}$ de $A$ telles que $h\left(B_{0}\right)=A_{0}$. 
Démonstration. - Soit $\left(B_{0} ;\left(B_{j}\right)_{j \in J}\right)$ une $\varepsilon$-approximation cylindrique de $B$. Par hypothèse, $B=\bigcup_{e, e^{\prime} \in \mathbf{N}}\left(B \cap \Delta_{e, e^{\prime}}\right)$ et $B_{0}=\bigcup_{e, e^{\prime} \in \mathbf{N}}\left(B_{0} \cap \Delta_{e, e^{\prime}}\right)$. Posons $B_{e, e^{\prime}}:=B \cap \Delta_{e, e^{\prime}}$ et $B_{e, e^{\prime}}^{(j)}:=B_{j} \cap \Delta_{e, e^{\prime}}$, pour tout $i \in J \cup\{0\}$. Par le lemme 7.1.3, l'ensemble $h\left(B_{e, e^{\prime}}^{(0)}\right)$ est un cylindre de $\operatorname{Gr}(X)$. En outre, le lemme 4.3.7 assure qu'il existe une famille finie d'éléments $\left(e_{i}, e_{j}^{\prime}\right) \in \mathbf{N}^{2}$ tels que

$$
B_{0}=\bigcup_{1 \leq i, j \leq m} B_{e_{i}, e_{j}^{\prime}}^{(0)}
$$

L'égalité $B \Delta B_{0} \subset \bigcup_{i \in J} B_{i}$ entraîne que $B_{e, e^{\prime}} \Delta B_{e, e^{\prime}}^{(0)} \subset \bigcup_{i \in J} B_{e, e^{\prime}}^{(i)}$ pour tout $e$ et $e^{\prime} \in \mathbf{N}$, et donc que $h\left(B_{e, e^{\prime}}\right) \Delta h\left(B_{e, e^{\prime}}^{(0)}\right) \subset \bigcup_{i \in J} h\left(B_{e, e^{\prime}}^{(i)}\right)$. Comme

$$
\bigcup_{e, e^{\prime} \in \mathbf{N}} h\left(B_{e, e^{\prime}}^{(0)}\right)=\bigcup_{1 \leq i, j \leq m} h\left(B_{e_{i}, e_{j}^{\prime}}^{(0)}\right),
$$

l'ensemble $h\left(B_{0}\right)$ est un cylindre de $\operatorname{Gr}(X)$. L'assertion 1) du lemme 7.2.1 assure la validité de l'assertion annoncée.

ThÉorÈme 7.2.3. - Soient $X$ et $Y$ des $\mathbb{D}$-schémas formels admissibles, tous deux purement de dimension relative d. Supposons que $Y \rightarrow \mathbb{D}$ est lisse. Soit $h: Y \rightarrow X$ un $\mathbb{D}$-morphisme tempéré de schémas formels. Si $B \subset \operatorname{Gr}(Y)$ est mesurable (resp. fortement mesurable), alors $h(B) \subset \operatorname{Gr}(X)$ est mesurable (resp. fortement mesurable).

Démonstration. - Soit $\varepsilon>0$. Posons $A:=h(B)$. On a :

$$
B=\left(B \cap \Sigma_{h}\right) \sqcup\left(B \backslash \Sigma_{h}\right) \text {. }
$$

Comme $h$ est tempéré, on peut supposer que $B \cap \Sigma_{h}=\varnothing$. Commençons par montrer que, pour tout $\varepsilon>0$, il existe une $\varepsilon$-approximation cylindrique de $B$ de partie principale contenue dans $\operatorname{Gr}(X) \backslash \Sigma_{h}$. Soit $\left(B_{0},\left(B_{j}\right)_{j \geq 1}\right)$ (resp. $\left.\left(\varnothing ;\left(\Sigma_{i}\right)_{i \geq 1}\right)\right)$ une $\varepsilon$-approximation cylindrique de $B\left(\operatorname{resp} . \Sigma_{h}\right)$. Comme l'ensemble $\Sigma_{h}$ est un pro-constructible de $\operatorname{Gr}(Y)$, la quasi-compacité de la topologie constructible entraîne que :

$$
\Sigma_{h} \subset \bigcup_{1 \leq i \leq m} \Sigma_{i}
$$

avec $m \geq 1$. La suite de cylindres de $\operatorname{Gr}(Y)$, définie par

$$
B_{0}^{\prime}:=B_{0} \backslash\left(\bigcup_{1 \leq i \leq m} \Sigma_{i}\right),
$$

les $\Sigma_{i}$ pour $1 \leq i \leq m$ et les $B_{j}$ pour $j \geq 1$, est une $\varepsilon$-approximation cylindrique de $B$. On peut donc supposer que $B_{0} \cap \Sigma_{h}=\varnothing$. Or

$$
h(B) \Delta h\left(B_{0}\right) \subset \bigcup_{i \geq 1} h\left(B_{i}\right) .
$$

BULletin DE LA SOCiÉtÉ MATHÉmATiQUE DE FRANCE 
Le théorème est alors une conséquence directe du lemme 7.2.1. La démonstration dans le cas où $B$ est fortement mesurable est identique. Remarquons que dans ce cas la condition $B_{0} \cap \Sigma_{h}=\varnothing$ est automatique, puisque on peut choisir $B_{0} \subset B$, par définition. L'inclusion $B_{0} \subset B$ entraîne également que $h\left(B_{0}\right) \subset A$ et l'assertion en découle.

7.3. La mesurabilité de l'image inverse. - Dans cette section, nous allons étudier le problème de la stabilité de la propriété de mesurabilité par image inverse via un morphisme $h$. Soit $h: Y \rightarrow X$ un $\mathbb{D}$-morphisme de schémas formels admissibles, tous deux purement de dimension relative $d$. Soit $A$ une partie mesurable de $\operatorname{Gr}(X)$. Rappelons que l'on désigne par

$$
\Delta_{e, e^{\prime}}=h^{-1}\left(\mathrm{Gr}^{\left(e^{\prime}\right)}(X)\right) \cap \operatorname{ord}_{\pi}\left(\mathrm{Jac}_{h}^{-1}(e) .\right.
$$

Dans ce paragraphe, nous étudions donc le problème de la mesurabilité de l'image inverse $h^{-1}(A)$.

Lemme 7.3.1. - Soit $A$ un cylindre de $\operatorname{Gr}(X)$ de rang $n$. Alors $h^{-1}(A)$ est un cylindre de $Y$ de rang $n$.

Démonstration. - Cela découle directement de la définition d'un cylindre et de la commutativité du diagramme

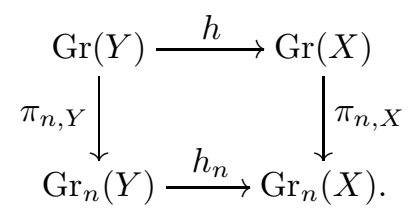

Supposons que le $\mathbb{D}$-morphisme de schémas formels $h$ est tempéré, que le $k$ morphisme de schémas $h: \operatorname{Gr}(Y) \rightarrow \operatorname{Gr}(X)$ est injectif et que $Y \rightarrow \mathbb{D}$ soit lisse.

ThÉORÈme 7.3.2. — Soient $X$ et $Y$ des $\mathbb{D}$-schémas formels admissibles, tous deux purement de dimension relative $d$. Supposons en outre que $Y \rightarrow \mathbb{D}$ est lisse. Soit $h: Y \rightarrow X$ un $\mathbb{D}$-morphisme tempéré de schémas formels induisant une application $h: \operatorname{Gr}(Y) \rightarrow \operatorname{Gr}(X)$ injective. Si A est mesurable (resp. fortement mesurable) dans $\operatorname{Gr}(X)$, alors l'ensemble $B:=h^{-1}(A)$ est mesurable (resp. fortement mesurable) dans $\operatorname{Gr}(Y)$.

Démonstration. - Soit $\left(A_{0} ;\left(A_{i}\right)_{i \geq 1}\right)$ une $\varepsilon$-approximation de $A$. Comme

$$
A=\left(A \cap \operatorname{Gr}\left(X_{\text {sing }}\right)\right) \sqcup\left(A \backslash \operatorname{Gr}\left(X_{\text {sing }}\right)\right),
$$

et comme $h$ est tempéré, on peut supposer que $A \cap \operatorname{Gr}\left(X_{\text {sing }}\right)=\varnothing$ et que $B \cap \Sigma_{h}=\varnothing$. Par définition,

$$
B \Delta h^{-1}\left(A_{0}\right) \subset \bigcup_{i \geq 1} h^{-1}\left(A_{i}\right) .
$$

TOME $132-2004-\mathrm{N}^{\mathrm{O}} 1$ 
Comme $\Sigma_{h}$ est de mesure nulle, il existe donc une famille de cylindres $(D(\delta))_{0<\delta<1}$, avec $D(\delta) \supset \Sigma_{h}$ et tel que

$$
D\left(\delta^{\prime}\right) \subset D(\delta) \text { si } \delta^{\prime} \leq \delta, \quad\left\|\mu_{Y}(D(\delta))\right\|<\delta, \quad \Sigma_{h}=\bigcap_{\delta} D(\delta) .
$$

On a donc

$$
(B \backslash D(\delta)) \Delta\left(h^{-1}\left(A_{0}\right) \backslash D(\delta)\right) \subset \bigcup_{i \geq 1}\left(h^{-1}\left(A_{i}\right) \backslash D(\delta)\right) .
$$

Par définition de $\Sigma_{h}$ et grâce au lemme 4.3.7, il existe une famille finie d'éléments $\left(e i, e_{j}^{\prime}\right) \in \mathbf{N}^{2}$ tels que $\operatorname{Gr}(Y) \backslash D(\delta) \subset \bigcup_{1 \leq i, j \leq m(\delta)} \Delta_{e_{i}, e_{j}^{\prime}}$. Par suite,

$$
\left\|\mu_{Y}\left(h^{-1}\left(A_{i}\right) \backslash D(\delta)\right)\right\| \leq \max _{1 \leq i, j \leq m(\delta)}\left(\left\|\mu_{Y}\left(\left(h^{-1}\left(A_{i}\right) \backslash D(\delta)\right) \cap \Delta_{e_{i}, e_{j}^{\prime}}\right)\right\|\right) .
$$

Il découle donc du lemme 7.1.3 que

$$
\left\|\mu_{Y}\left(h^{-1}\left(A_{i}\right) \backslash D(\delta)\right)\right\| \leq \max 2^{e_{i}}\left\|\mu_{X}\left(A_{i}\right)\right\| .
$$

Posons $r(\delta):=\max \left(2^{e_{i}}\right)$. La suite de cylindres de $\operatorname{Gr}(Y)$, définie par $\left(h^{-1}\left(A_{i}\right) \backslash D(\delta)\right)$, est une $(r(\delta) \times \varepsilon)$-approximation cylindrique de $B$. Pour tout $\delta>0$, l'ensemble $B \backslash D(\delta)$ est donc mesurable. Il nous faut en déduire que $B$ lui-même est mesurable. Soit $\left(\delta_{n}\right)_{n \in \mathbf{N}}$ une suite strictement décroissante d'entiers compris entre 0 et 1 . L'égalité

$$
\left(B \backslash D\left(\delta_{0}\right)\right) \sqcup\left(\bigsqcup_{n \geq 1}\left(B \cap D\left(\delta_{n}\right) \backslash D\left(\delta_{n+1}\right)\right)=B \backslash \Sigma_{h}=B\right.
$$

permet de conclure ( $c f$. proposition 6.3.4). Il découle de la construction et de la proposition 6.3.4 que, si $A$ est fortement mesurable, l'ensemble $B$ est également fortement mesurable.

\section{Théorème de changement de variables}

Proposition 8.0.3. - Soient $X$ et $Y$ deux $\mathbb{D}$-schémas formels admissibles, purement de dimension relative $d$. Supposons que $Y \rightarrow \mathbb{D}$ est lisse. Soient $A$ et $B$ des ensembles fortement mesurables de $\operatorname{Gr}(X)$ et $\operatorname{Gr}(Y)$ respectivement. Soit $h: Y \rightarrow X$ un $\mathbb{D}$-morphisme de schémas formels tempéré sur $B$ qui induit une bijection entre $B$ et $A$. Alors, pour toute application exponentiellement intégrable $\alpha: A \rightarrow \mathbf{Z} \cup\{\infty\}$, l'application $B \rightarrow \mathbf{Z} \cup\{\infty\}$ qui à y associe $\alpha(h(y))+\operatorname{ord}_{\pi}(\mathrm{Jac})(y)$ est exponentiellement intégrable et on a dans $\widehat{M}_{k}$ l'égalité

$$
\int_{A} \mathbf{L}^{-\alpha} \mathrm{d} \mu=\int_{B} \mathbf{L}^{-\alpha \circ h-\operatorname{ord}_{\pi}\left(\mathrm{Jac}_{h}\right.} \mathrm{d} \mu .
$$

BULletin DE LA SOCIÉtÉ MATHÉMATIQUE DE FRANCE 
Démonstration. - Soit $\varepsilon>0$. Soit $\left(A_{0} ;\left(A_{i}\right)_{i \geq 1}\right)\left(\operatorname{resp.}\left(B_{0} ;\left(B_{j}\right)_{j \geq 1}\right)\right)$ une $\varepsilon$-appro-ximation cylindrique de $A$ (resp. $B)$. Comme $h$ est tempéré sur $B$, on peut supposer que $B \cap \Sigma_{h}=\varnothing$. Prouvons tout d'abord que l'application $\beta:=\alpha \circ h+\operatorname{ord}_{\pi}(\mathrm{Jac})_{h}$ est mesurable sur $B$. Soit $n \in \mathbf{Z}$. Soit $\left(F_{0}^{(n)} ;\left(F_{\ell}^{(n)}\right)_{\ell \geq 1}\right)$ une $\varepsilon$-approximation cylindrique de $\alpha^{-1}(n) \subset A$. Nous allons montrer que $(\alpha \circ h)^{-1}(n) \cap B$ est un ensemble mesurable de $\operatorname{Gr}(Y)$. On peut supposer que $F_{0}^{(n)} \subset A_{0} \subset A(c f$. lemme 6.3.1). On a l'égalité

$$
\left(B \cap h^{-1}\left(\alpha^{-1}(n)\right)\right) \Delta\left(B \cap h^{-1}\left(F_{0}^{(n)}\right)\right) \subset \bigcup_{i \geq 1} h^{-1}\left(F_{i}^{(n)}\right) .
$$

Grâce au corollaire 7.2.2 et à l'injectivité de $h$ sur $B$, on peut supposer que $h^{-1}\left(A_{0}\right) \cap B=B_{0}$. En particulier, cette remarque entraîne que

$$
\left(h^{-1}\left(\alpha^{-1}(n)\right) \cap B\right) \Delta\left(h^{-1}\left(F_{0}^{(n)}\right) \cap B\right) \subset\left(\bigcup_{j \geq 1} B_{i}\right) \cup\left(\bigcup_{i \geq 1} h^{-1}\left(F_{i}^{(n)}\right) \cap B_{0}\right) .
$$

En effet, par hypothèse, on a

$$
\begin{gathered}
\left(h^{-1}\left(\alpha^{-1}(n)\right) \cap B\right) \backslash B_{0} \subset B \backslash B_{0}, \\
\left(h^{-1}\left(\alpha^{-1}(n)\right) \backslash h^{-1}\left(F_{0}^{(n)}\right)\right) \cap B_{0} \subset \bigcup_{i \geq 1} h^{-1}\left(F_{i}^{(n)}\right) \cap B_{0},
\end{gathered}
$$

et, comme $h^{-1}\left(F_{0}^{(n)}\right) \cap B \subset B_{0}$,

$$
\left(B \cap h^{-1}\left(F_{0}^{(n)}\right)\right) \backslash h^{-1}\left(\alpha^{-1}(n)\right) \subset \bigcup_{i \geq 1} h^{-1}\left(F_{i}^{(n)}\right) \cap B_{0} .
$$

Le lemme 7.1.3, 2) assure alors que $B \cap(\alpha \circ h)^{-1}(n)$ est mesurable. Comme

$$
\beta^{-1}(n)=\bigsqcup_{e \in \mathbf{N}}\left((\alpha \circ h)^{-1}(n-e) \cap \operatorname{ord}_{\pi}(\mathrm{Jac})_{h}^{-1}(e) \cap B\right),
$$

la suite $\left(\mu_{Y}\left(\operatorname{ord}_{\pi}(\mathrm{Jac})_{h}^{-1}(e) \cap B\right)_{e \in \mathbf{N}}\right.$ tend vers 0 . La proposition 6.3.4 assure alors que l'application $\alpha \circ h+\operatorname{ord}_{\pi}(\mathrm{Jac})_{h}$ est mesurable sur $B$.

Il s'agit de montrer maintenant que l'intégrale converge et qu'on a bien la formule annoncée. Le lemme 4.3.7 assure qu'il existe un entier $r \in \mathbf{N}$ tel que

$$
B_{0} \subset \bigcup_{e, e^{\prime} \leq r} \Delta_{e, e^{\prime}}
$$

Par additivité de l'intégrale, il suffit de montrer le résultat pour $B_{0} \subset \Delta_{e, e^{\prime}}$, pour un entier $e \in \mathbf{N}$ et un entier $e^{\prime} \in \mathbf{N}$. Soit $\varepsilon>0$. Soit $n \in \mathbf{Z}$. Notons $\left(G_{0}^{(n-e)} ;\left(G_{m}^{(n-e)}\right)_{m \geq 1}\right)$ une $\varepsilon$-approximation cylindrique de $B \cap(\alpha \circ h)^{-1}(n-e)$, qui est mesurable dans $\operatorname{Gr}(X)$, telle que $G_{0}^{(n-e)} \subset B_{0}$. On peut supposer que $F_{0}^{(n-e)}=h\left(G_{0}^{(n-e)}\right)$. Le lemme 7.1 .3 assure alors que

$$
\left[\pi_{\nu, Y}\left(G_{0}^{(n-e)}\right)\right] \mathbf{L}^{-\nu} \mathbf{L}^{-n}=\left[\pi_{\nu, X}\left(F_{0}^{(n-e)}\right)\right] \mathbf{L}^{-\nu} \mathbf{L}^{-n+e} .
$$

TOME $132-2004-\mathrm{N}^{\mathrm{O}} 1$ 
Autrement dit,

$$
\mu_{Y}\left(\left(\alpha \circ h+\operatorname{ord}_{t}(\mathrm{Jac})_{h}\right)^{-1}(n) \cap B\right) \mathbf{L}^{-n}=\mu_{Y}\left(\alpha^{-1}(n-e)\right) \mathbf{L}^{-(n-e)} .
$$

La formule annoncée en découle.

Grâce à la théorie des modèles de Néron faibles en géométrie formelle ( $c f$. [8], déf. 1.3 et th. 3.1), on peut généraliser cette proposition au cas où seule la fibre générique de $Y$ est supposée lisse sur $K$. Commençons par reformuler en termes de schéma de Greenberg l'existence de modèles de Néron faibles en géométrie formelle (cf. loc. cit. pour la définition originale) :

ThÉORÈme 8.0.4. - Soit $X$ est un $\mathbb{D}$-schéma formel admissible, de fibre générique $X_{K}$ lisse sur $K$. Il existe un $\mathbb{D}$-morphisme de schémas formels $g: U \rightarrow X$ tempéré induisant une bijection $\operatorname{Gr}(U) \rightarrow \operatorname{Gr}(X)$ et tel que $U$ est quasi-compact et lisse sur $\mathbb{D}$.

Démonstration. — L'idée de la démonstration est l'utilisation des modèles de Néron faibles. Ces modèles peuvent s'obtenir en considérant le lieu lisse d'un $\mathbb{D}$-schéma formel $X^{\prime}$ obtenu à partir de $X$ par éclatements admissibles ( $c f$. [8], th. 3.1). Par suite, un tel morphisme $g$ est tempéré.

ThÉorème 8.0.5. - Soient $X$ et $Y$ deux $\mathbb{D}$-schémas formels admissibles, purement de dimension relative $d$. Supposons que $Y$ soit génériquement lisse. Soient $B$ et $A$ des ensembles fortement mesurables de $\operatorname{Gr}(Y)$ et $\operatorname{Gr}(X)$ respectivement. Soit $h: Y \rightarrow X$ un $\mathbb{D}$-morphisme de schémas formels tempéré sur $B$ qui induit une bijection entre $B$ et $A$. Soit $\alpha: A \rightarrow \mathbf{Z} \cup\{\infty\}$ une application exponentiellement intégrable. Alors l'application qui à $y \in B$ associe $\alpha \circ h(y)+\operatorname{ord}_{\pi}(\mathrm{Jac})_{h}(y)$ est exponentiellement intégrable et l'on a dans $\widehat{M}_{k}$ l'égalité

$$
\int_{A} \mathbf{L}^{-\alpha} \mathrm{d} \mu_{X}=\int_{B} \mathbf{L}^{-\left(\alpha \circ h+\operatorname{ord}_{\pi}\left(\mathrm{Jac}_{h}\right)\right.} \mathrm{d} \mu_{Y}
$$

Démonstration. - On peut supposer, comme dans la preuve de 8.0.3, que $B \cap \Sigma_{h}=\varnothing$ et que $A \cap \operatorname{Gr}\left(X_{\text {sing }}\right)=\varnothing$. La théorie des modèles de Néron faibles ( $c f$. théorème 8.0.5) assure qu'il existe un ouvert $U \rightarrow \mathbb{D}$, lisse et quasi-compact, d'un $\mathbb{D}$-modèle de la fibre générique de $Y$, notée $Y_{K}$, et un morphisme $g: U \rightarrow Y$ induisant une bijection entre les points de $\operatorname{Gr}(U)$ et ceux de $\operatorname{Gr}(Y)$. On a alors un diagramme commutatif :

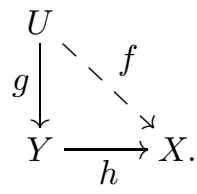

BULLETIN DE LA SOCIÉtÉ MATHÉMATIQUE DE FRANCE 
Posons $C:=g^{-1}(B)$. Le morphisme $g$ induit une bijection entre $C$ et $B$ et 7.3 .2 assure que $g^{-1}(B)=: C$ est fortement mesurable. Comme $g$ est tempéré, on peut supposer que $C \cap \Sigma_{g}=\varnothing$. Il en résulte tout d'abord que

$$
C \cap g^{-1}\left(h^{-1}\left(\operatorname{Gr}\left(X_{\text {sing }}\right)\right)\right)=\varnothing .
$$

Par ailleurs, la formule de composition ( $c f$. proposition 5.1.7) assure alors que $C \cap \Pi_{f}=\varnothing$. On a donc $C \cap \Sigma_{f}=\varnothing$. Il faut prouver que l'application $\alpha \circ h+\operatorname{ord}_{\pi}(\mathrm{Jac})_{h}$ est exponentiellement intégrable sur $B$. Soit $F_{n}=(\alpha \circ$ $\left.h+\operatorname{ord}_{\pi}(\mathrm{Jac})_{h}\right)^{-1}(n)$. On remarque que

$$
F_{n}=g\left(\bigsqcup_{e \in \mathbf{N}}(\alpha \circ h \circ g)^{-1}(n-e) \cap\left(\operatorname{ord}_{\pi}(\mathrm{Jac})_{h} \circ g\right)^{-1}(e) \cap C\right) .
$$

Or la formule de composition 5.1.7 assure que

$$
\left(\operatorname{ord}_{\pi}(\mathrm{Jac})_{h} \circ g\right)^{-1}(e)=\bigsqcup_{\text {finie }}\left(\left(\operatorname{ord}_{\pi}(\mathrm{Jac})_{f}\right)^{-1}\left(e-e^{\prime}\right) \cap\left(\operatorname{ord}_{\pi}(\operatorname{Jac})_{g}\right)^{-1}\left(e^{\prime}\right)\right) .
$$

La mesurabilité de $F_{n}$ se déduit, comme dans la preuve de 8.0.3, de la mesurabilité de $\alpha$ et du théorème 7.2.3. La convergence de l'intégrale découle alors du lemme 7.1.3, comme dans la preuve du théorème 8.0.3.

Supposons maintenant que l'application $\alpha \circ h+\operatorname{ord}_{\pi}(\mathrm{Jac})_{h}$ est exponentiellement intégrable sur $B$. Le théorème 8.0.3 implique alors les égalités dans $\widehat{M}_{k}$

$$
\begin{aligned}
\int_{A} \mathbf{L}^{-\alpha} d \mu_{X} & =\int_{C} \mathbf{L}^{-\alpha \circ h \circ g-\operatorname{ord}_{\pi}(\mathrm{Jac})_{h \circ g} \mathrm{~d} \mu_{U},} \\
\int_{B} \mathbf{L}^{-\alpha \circ h-\operatorname{ord}_{\pi}\left(\mathrm{Jac}_{h}\right.} d \mu_{Y} & =\int_{C} \mathbf{L}^{-\alpha \circ h \circ g-\operatorname{ord}_{\pi}\left(\mathrm{Jac}_{h} \circ g-\operatorname{ord}_{\pi}(\mathrm{Jac})_{g}\right.} \mathrm{d} \mu_{U} .
\end{aligned}
$$

La formule découle alors de la proposition 5.1.7.

\section{BIBLIOGRAPHIE}

[1] BAtyrev (V.) - Stringy Hodge numbers of varieties with Gorenstein canonical singularities, in Integrable systems and algebraic geometry (Kobe/Kyoto, 1997), World Sci. Publishing, 1998, pp. 1-32.

[2] Birational Calabi-Yau n-folds have equal Betti numbers, in New trends in algebraic geometry (Warwick, 1996), London Math. Soc. Lecture Note Ser., vol. 264, Cambridge Univ. Press, 1999, pp. 1-11.

[3] Bosch (S.), Güntzer (U.) \& Remmert (R.) - Non-Archimedean analysis, Grundlehren Math., vol. 261, Springer-Verlag, 1984.

[4] Bosch (S.) \& Lütкebohmert (W.) - Formal and rigid geometry. I. Rigid spaces, Math. Ann., t. 295 (1993), no. 2, pp. 291-317.

[5] , Formal and rigid geometry. II. Flattening techniques, Math. Ann., t. 296 (1993), no. 3, pp. 403-429.

TOME $132-2004-\mathrm{N}^{\mathrm{O}} 1$ 
[6] Bosch (S.), Lütkebohmert (W.) \& Raynaud (M.) - Néron models, Ergebnisse Math., vol. 21, Springer-Verlag, 1990.

[7] Formal and rigid geometry. III. The relative maximum principle, Math. Ann., t. 302 (1995), no. 1, pp. 1-29.

[8] Bosch (S.) \& Schlöter (K.) - Néron models in the setting of formal and rigid geometry, Math. Ann., t. 301 (1995), no. 2, pp. 339-362.

[9] Bourbaki (N.) - Éléments de mathématique. Fascicule XXVIII. Algèbre commutative. Chapitre 3 : Graduations, filtrations et topologies. Chapitre 4 : Idéaux premiers associés et décomposition primaire, Hermann, 1961.

[10] Denef (J.) \& Loeser (F.) - Germs of arcs on singular algebraic varieties and motivic integration, Invent. Math., t. 135 (1999), no. 1, pp. 201-232.

[11] _ Geometry on arc spaces of algebraic varieties, in European Congress of Mathematics, Vol. I (Barcelona, 2000), Progr. Math., vol. 201, Birkhäuser, 2001, pp. 327-348.

[12] _ Motivic integration, quotient singularities and the McKay correspondence, Compositio Math., t. 131 (2002), no. 3, pp. 267-290.

[13] Greenberg (M.J.) - Schemata over local rings, Ann. of Math., t. 73 (1961), no. 2, pp. 624-648.

[14] Rational points in Henselian discrete valuation rings, Inst. Hautes Études Sci. Publ. Math., t. 31 (1966), pp. 59-64.

[15] Grothendieck (A.) - Éléments de géométrie algébrique. IV. Étude locale des schémas et des morphismes de schémas. III, Inst. Hautes Études Sci. Publ. Math., t. 28 (1966).

[16] Grothendieck (A.) \& Dieudonné (J.A.) - Éléments de géométrie algébrique. I, Grundlehren Math., vol. 166, Springer-Verlag, 1971.

[17] Kapranov (M.) \& Vasserot (E.) - Vertex algebras and the formal loop space, AG/0107143.

[18] Kontsevich (M.) - Lecture at Orsay, December 7, 1995.

[19] Loeser (F.) \& Sebag (J.) - Motivic integration on smooth rigid varieties and invariants of degenerations, AG/0107134 (à paraître dans Duke Math. J.).

[20] LooiJenga (E.) - Motivic measures, in Séminaire Bourbaki 1999/2000, Astérisque, vol. 276, 2002, pp. 267-297.

[21] Oesterlé (J.) - Réduction modulo $p^{n}$ des sous-ensembles analytiques fermés de $\mathbf{Z}_{p}^{N}$, Invent. Math., t. 66 (1982), no. 2, pp. 325-341.

[22] Poonen (B.) - The Grothendieck ring of varieties is not a domain, Math. Res. Lett., t. 9 (2002), no. 4, pp. 493-497.

[23] Raynaud (M.) - Géométrie analytique rigide d'après Tate, Kiehl..., in Table Ronde d'Analyse non archimédienne (Paris, 1972), Mémoires, vol. 39-40, Soc. Math. France, 1974, pp. 319-327. 
[24] Schappacher (N.) - Some remarks on a theorem of M.J. Greenberg, in Proceedings of the Queen's Number Theory Conference, 1979 (Kingston, Ont., 1979), Queen's Papers in Pure and Appl. Math., vol.54, Queen's Univ., 1980, pp. 101-113.

[25] SERRE (J-P.) - Classification des variétés analytiques p-adiques compactes, Topology, t. 3 (1965), pp. 409-412.

[26] Swan (R.G.) - Néron-Popescu desingularization, in Algebra and geometry (Taipei, 1995), Lect. Algebra Geom., vol. 2, Internat. Press, 1998, pp. 135192. 